\title{
Numerical Interpretation of Experimental Data on Aerodynamics of the HB-2 Model Using Computer Codes USTFEN and PERAT-3D
}

\author{
Sergey T. Surzhikov \\ Ishlinskyi Institute for Problems in Mechanics Russian Academy of Sciences (IPMech RAS), \\ Moscow, 119526, Russia \\ N. L. Dukhov All-Russian Scientific Research Institute of Automatics \\ surg@ipmnet.ru
}

\begin{abstract}
The results of validation and cross-verification of two computer codes intended for spatial calculations of supersonic flow of perfect viscous and heat-conducting gas around bodies of complex geometry are presented. We used two in-home computer codes designed for the numerical integration of the Navier-Stokes and Euler equations on tetrahedral unstructured and multi-block structured computational grids. The government relations underlying both codes are analyzed in detail and the features of their use in numerical implementation are discussed.
\end{abstract}

Keywords: hypersonic flow, validation of copyright computer codes
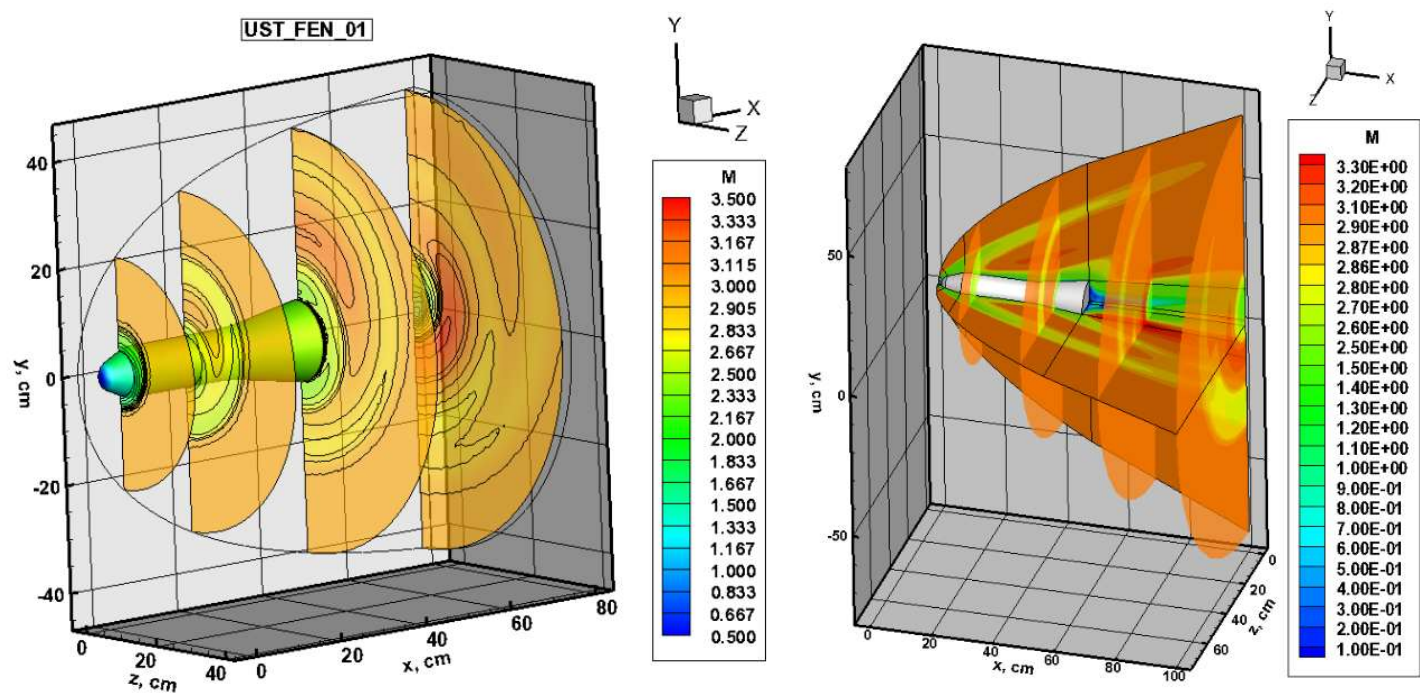

Mach numbers distribution around experimental ballistic model HB-2 at $\mathrm{M}=3$ and $\mathrm{AoA}=8^{\circ}$ 


\title{
Численная интерпретация экспериментальных данных по аэродинамике модели НВ-2 \\ с использованием компьютерных кодов USTFEN и PERAT-3D
}

\author{
С.Т. Суржиков \\ Институт проблем механики им. А. Ю. Иилинского Российской академии наук \\ Россия, Москва, 119526, проспект Вернадского, д. 101-1
}

Всероссийский научно-исследовательский институт автоматики им. Н. Л. Духова surg@ipmnet.ru

\begin{abstract}
Аннотация
Представлены результаты валидации и перекрестной верификации двух авторских компьютерных кодов, предназначенных для пространственных расчетов обтекания тел сложной геометрии сверхзвуковыми потоками совершенного вязкого и теплопроводного газа. Использовались два авторских компьютерных кода, предназначенных для численного интегрирования уравнений Навье - Стокса и Эйлера на тетраэдральных неструктурированных и многоблочных структурированных расчетных сетках. Подробно анализируются расчетные соотношения, положенные в основу обоих кодов и обсуждаются особенности их использования при численной реализации.
\end{abstract}

Ключевые слова: гиперзвуковое течение, валидация авторских компьютерных кодов.

\section{1. Введение}

Проблема верификации и валидации компьютерных кодов является одной из наиболее важных в развитии вычислительных методов механики жидкости и газа. Особую значимость она приобретает при разработке численных моделей и реализующих их компьютерных программ (далее, для краткости - кодов) для задач аэротермодинамики и аэрофизики гиперзвуковых скоростей. Это связано с тем, что при гиперзвуковых скоростях уменьшаются характерные размеры газодинамических структур (в частности, пограничных слоев) и увеличиваются градиенты газодинамических функций, а также начинают проявляться реальные свойства высокотемпературного газа, включая химические реакции, диссоциация, ионизация и даже радиационный перенос энергии. Информативные эксперименты для таких условий выполнить очень сложно и, в большинстве случаев, пока невозможно. Поэтому в последнее десятилетие наметилась тенденция постановки летных экспериментов, которым предшествуют серии наземных стендовых испытаний и подробный численный анализ их результатов [1-11].

Несмотря на значительные затрачиваемые усилия следует подчеркнуть, что число надежных и хорошо документированных данных чрезвычайно мало. Это сдерживает не только уверенное проектирование перспективных летательных аппаратов, но и развитие фундаментальных направлений гиперзвуковой аэротермодинамики и аэрофизики.

В данной работе представлены результаты тестирования двух авторских компьютерных кодов, которые продолжают непрерывно развиваться с целью решения задач фундаментального и прикладного характера аэротермодинамики и аэрофизики гиперзвуковых течений.

В предшествующих работах автора были представлены результаты верификации и валидации ряда задач. В работе [12] представлены результаты валидации авторского компьютер- 
ного кода UST3D (UnStructured Tetrahedral 3-Dimensional) на примере анализа экспериментальных данных по аэротермодинамике гиперзвуковых летательных аппаратов X-33 и X-34, a также затупленного по сфере конуса. Продемонстрировано вполне хорошее совпадение с опытными данными для летательных аппаратов, для которых форма поверхности известна не абсолютно точно, и полное соответствие опытным данным для простой модели затупленного конуса.

В работе [13] на примере экспериментальных данных по сверхзвуковому обтеканию затупленного по сфере конуса выполнено сравнение результатов расчетов, полученных по двум расчетным кодам, аналогичным исследуемым в настоящей работе. Особое внимание обращено на интерпретации численных данных по течению в отрывной зоне и при использовании вариаций форм исходных уравнений Навье-Стокса (точнее, по способам интегрирования уравнения сохранения энергии совместно с системой уравнений Навье-Стокса).

В работах [14-16] выполнено систематическое исследование целого ряда экспериментальных задач, связанных с разработкой возвращаемых космических аппаратов. Использовались структурированные расчетные сетки и, насколько это было возможно, полные модели термодинамических и теплофизических свойств, включая расчет физической и химической кинетики и перенос селективного теплового излучения.

В работе [17] дана численная интерпретация экспериментальных данных по нагреву поверхности исследовательского гиперзвукового аппарата HIFiRE-1, включая учет ламинарнотурбулентного перехода на поверхности.

В работе [18] проведено систематическое исследование закономерностей ударно-волнового взаимодействия с ламинарным пограничным слоем при гиперзвуковом обтекании поверхностей с изломом образующей.

Первый из исследуемых в настоящей работе компьютерных кодов, USTFEN, является развитием серии компьютерных кодов UST3D [19, 20], предназначенных для интегрирования уравнений Эйлера и Навье - Стокса на неструктурированных тетраэдральных расчетных сетках. Специфической особенностью данного кода является использование уравнения для полной удельной энергии газового потока в различных вариациях реализации при численном моделировании схем расщепления [21-23].

Компьютерные коды данной серии успешно использовались в работах [24-29]. Заметим, что во всех указанных работах выполнено развитие базового кода в части изучения эффективности различных схем расчета распада разрыва на гранях ячеек. Технологической особенностью реализации данного кода является использование технологии распараллеливания OpenMP и усовершенствованного сеточного препроцессора, осуществляющего переформатирование исходных сеточных данных в формате 'neu' к формату их более эффективного использования в основном блоке интегрирования на неструктурированных расчетных тетраэдральных сетках.

Второй из исследованных кодов, PERAT-3D, является упрощенной версией компьютерной программы NERAT-3D, реализующей интегрирование пространственных уравнений Навье-Стокса для химически неравновесного и излучающего газа на структурированных многоблочных сетках. В данном случае изучается расчетная модель термически совершенного вязкого и теплопроводного газа. Примеры верификационных и валидационных исследований кода PERAT-3D представлены в работах [14-16]. Для работы компьютерного кода PERAT-3D также требуется сеточный препроцессор, в котором с использованием аналитических отображений создаются многоблочные неоднородные структурированные расчетные сетки.

Предметом изучения данной работы являются аэродинамические характеристики затупленного по сфере кругового цилиндра с юбкой конуса конечного размера, обтекание которого экспериментально изучалось в [30].

Указанная предельно простая конфигурация аэродинамической модели позволяет изучить многие характерные черты течения, наблюдаемые у поверхности высокоскоростных летательных аппаратов. 
Данная работа преследует следующие цели:

- $\quad$ валидация компьютерных кодов USTFEN и PERAT-3D на примере экспериментальных данных [30];

- $\quad$ сравнение результатов расчета аэродинамических характеристик затупленного по сфере кругового цилиндра с юбкой для условий эксперимента [30], полученных с использованием разных компьютерных кодов на структурированных и неструктурированной сетке (так называемая перекрестная верификация компьютерных кодов);

- $\quad$ изучение различий в расчетных данных по структуре обтекания и отрывного течения.

Еще одной технологической проблемой является отработка способов графического представления результатов расчетов. Ниже будет показано, что существует многообразие форматов представления расчетных данных. Очевидно, что оптимальный способ должен быть определен в результате подробного обсуждения данного вопроса.

Данная работа состоит из пяти частей. В первой части сформулирована система Навье Стокса в удобном для численного интегрирования виде и изложен алгоритм численного моделирования на неструктурированных тетраэдральных сетках.

Во второй части дана формулировка трехмерного (3D) конечно-объемного метода для тетраэдральной сетки.

Во третьей части работы приведены расчетные соотношения, используемые при численном интегрировании на тетраэдральных сетках. PERAT-3D.

В четвертой части дана формулировка уравнений, реализуемых в компьютерном коде

В заключительной, пятой части работы, анализируются и сравниваются между собой полученные расчетные данные на структурированных и неструктурированных расчетных сетках. Выполнено сравнение по ряду аэродинамических характеристик с экспериментальными данными.

\section{2. Система уравнений и алгоритм численного интегрирования, используемые в компьютерном коде USTFEN}

Систему уравнений неразрывности, Навье - Стокса и сохранения полной энергии термически совершенного газа представим в векторно-матричном виде

$$
\begin{gathered}
\frac{\partial \mathbf{U}}{t}+\frac{\partial}{\partial x}\left(\mathbf{F}_{\rho V}^{x}+\mathbf{F}_{p}^{x}-\mathbf{T}_{\tau}^{x}\right)+\frac{\partial}{\partial y}\left(\mathbf{F}_{\rho V}^{y}+\mathbf{F}_{p}^{y}-\mathbf{T}_{\tau}^{y}\right)+\frac{\partial}{\partial z}\left(\mathbf{F}_{\rho V}^{z}+\mathbf{F}_{p}^{z}-\mathbf{T}_{\tau}^{z}\right)=\mathbf{R}, \\
\mathbf{U}=\left[\begin{array}{c}
\rho \\
\rho u \\
\rho v \\
\rho w \\
\rho E
\end{array}\right]=\left[\begin{array}{c}
\rho \\
\rho u \\
\rho v \\
\rho w \\
\left.e+0.5\left(u^{2}+v^{2}+w^{2}\right)\right]
\end{array}\right], \quad \mathbf{R}=\left[\begin{array}{c}
0 \\
\rho f_{x} \\
\rho f_{y} \\
\rho f_{z} \\
Q_{\Sigma}
\end{array}\right], \\
\mathbf{F}_{\rho V}^{x}=\left[\begin{array}{l}
\rho u \\
\rho u^{2} \\
\rho u v \\
\rho u w \\
\rho u E
\end{array}\right], \mathbf{F}_{\rho V}^{y}=\left[\begin{array}{l}
\rho v \\
\rho v u \\
\rho v^{2} \\
\rho v w \\
\rho v E
\end{array}\right], \mathbf{F}_{\rho V}^{y}=\left[\begin{array}{l}
\rho w \\
\rho w u \\
\rho w v \\
\rho w^{2} \\
\rho w E
\end{array}\right],
\end{gathered}
$$




$$
\begin{gathered}
\mathbf{F}_{p}^{x}=\left[\begin{array}{c}
0 \\
p \\
0 \\
0 \\
p u+q_{x}
\end{array}\right], \mathbf{F}_{p}^{y}=\left[\begin{array}{c}
0 \\
0 \\
p \\
0 \\
p v+q_{y}
\end{array}\right], \mathbf{F}_{p}^{z}=\left[\begin{array}{c}
0 \\
0 \\
0 \\
0 \\
p \\
\tau_{y x} \\
\tau_{x x}+q_{z}
\end{array}\right], \mathbf{T}_{\tau}^{y}=\left[\begin{array}{c}
\tau_{y y} \\
\tau_{y z} \\
\tau_{x z} \\
u \tau_{x x}+v \tau_{x y}+w \tau_{x z}
\end{array}\right], \mathbf{T}_{\tau}^{z}=\left[\begin{array}{c}
0 \\
\tau_{z x} \\
\tau_{z y} \\
\tau_{z z} \\
u \tau_{y x}+v \tau_{y y}+w \tau_{y z}
\end{array}\right],
\end{gathered}
$$

где $u, v, w$ - проекции скорости $\vec{V}$ на координатные оси $x, y, z ; \rho, p$ - плотность и давление; $E=\rho\left(e+V^{2} / 2\right)-$ полная внутренняя энергия единицы объема; $e-$ удельная внутренняя энергия; $f_{x}, f_{y}, f_{z}$ - массовые объемные силы; $q_{x}, q_{y}, q_{z}$ - проекции вектора плотности теплового потока q на координатные оси; $Q_{\Sigma}$ - объемные источники тепловыделения; $\tau_{i j}-$ компоненты тензора вязких напряжений, являющиеся составными частями компонентов тензора напряжений

$$
\Pi_{i j}=-p \delta_{i j}+\tau_{i j}
$$

где $\delta_{i j}$ - дельта-символ Кронекера ( $\delta_{i j}=1$, если $i=j ; \delta_{i j}=0$, если $\left.i \neq j\right)$, имеют следующий вид:

$$
\begin{gathered}
\tau_{x x}=\frac{2}{3} \mu\left[2 \frac{\partial u}{\partial x}-\left(\frac{\partial v}{\partial y}+\frac{\partial w}{\partial z}\right)\right], \tau_{y y}=\frac{2}{3} \mu\left[2 \frac{\partial v}{\partial y}-\left(\frac{\partial u}{\partial x}+\frac{\partial w}{\partial z}\right)\right], \tau_{z z}=\frac{2}{3} \mu\left[2 \frac{\partial w}{\partial z}-\left(\frac{\partial u}{\partial x}+\frac{\partial v}{\partial y}\right)\right] \\
\tau_{x y}=\tau_{y x}=\mu\left(\frac{\partial u}{\partial y}+\frac{\partial v}{\partial x}\right), \tau_{x z}=\tau_{z x}=\mu\left(\frac{\partial w}{\partial x}+\frac{\partial u}{\partial z}\right), \tau_{y z}=\tau_{z y}=\mu\left(\frac{\partial v}{\partial z}+\frac{\partial w}{\partial y}\right)
\end{gathered}
$$

Термическое и калорическое уравнения состояния совершенного газа

$$
p=(\gamma-1) \rho e, e=c_{V} T,
$$

где $T$ - температура; $c_{V}$ - удельная теплоемкость при постоянном объеме.

При интегрировании уравнений движения невязкого и нетеплопроводного газа компоненты тензора вязких напряжений и теплового потока полагались равными нулю.

Граничные условия для компонент скорости формулировались либо в форме прилипания, либо в форме проскальзывания (нормальная компонента скорости равна нулю). Далее полагаем компоненты векторной функции $\mathbf{R}$ равными нулю.

Использовался двухшаговый алгоритм интегрирования системы уравнений (1). Для удобства формулировки алгоритма представим (1) в следующем символическом виде (фактически используется схема расщепления по физическим процессам), вводя шаг интегрирования по времени $\tau$

$$
\frac{\mathbf{U}^{p+1}-\tilde{\mathbf{U}}}{\tau}+\frac{\tilde{\mathbf{U}}-\mathbf{U}^{p}}{\tau}+\frac{\partial}{\partial x}\left(\mathbf{F}_{\rho V}^{x}\right)+\frac{\partial}{\partial y}\left(\mathbf{F}_{\rho V}^{y}\right)+\frac{\partial}{\partial z}\left(\mathbf{F}_{\rho V}^{z}\right)+\frac{\partial}{\partial x}\left(\mathbf{F}_{p}^{x}-\mathbf{T}_{\tau}^{x}\right)+\frac{\partial}{\partial y}\left(\mathbf{F}_{p}^{y}-\mathbf{T}_{\tau}^{y}\right)+\frac{\partial}{\partial z}\left(\mathbf{F}_{p}^{z}-\mathbf{T}_{\tau}^{z}\right)=0
$$


или, последовательно, на шаге "предиктор" для компонент скорости и полной удельной энергии

$$
\frac{\tilde{\mathbf{U}}-\mathbf{U}^{p}}{\tau}+\frac{\partial}{\partial x}\left(\mathbf{F}_{p}^{x}-\mathbf{T}_{\tau}^{x}\right)+\frac{\partial}{\partial y}\left(\mathbf{F}_{p}^{y}-\mathbf{T}_{\tau}^{y}\right)+\frac{\partial}{\partial z}\left(\mathbf{F}_{p}^{z}-\mathbf{T}_{\tau}^{z}\right)=0,
$$

а на шаге "корректор"

$$
\frac{\mathbf{U}^{p+1}-\tilde{\mathbf{U}}}{\tau}+\frac{\partial}{\partial x}\left(\tilde{\mathbf{F}}_{\rho V}^{x}\right)+\frac{\partial}{\partial y}\left(\tilde{\mathbf{F}}_{\rho V}^{y}\right)+\frac{\partial}{\partial z}\left(\tilde{\mathbf{F}}_{\rho V}^{z}\right)=0
$$

Покомпонентная запись формул для шага "предиктор"

$$
\begin{gathered}
\tilde{u}=u^{p}-\frac{\tau}{\rho^{p}} \frac{\partial p}{\partial x}+\frac{\tau}{\rho^{p}}\left(\frac{\partial \tau_{x x}}{\partial x}+\frac{\partial \tau_{x y}}{\partial y}+\frac{\partial \tau_{x z}}{\partial z}\right), \\
\tilde{v}=v^{p}-\frac{\tau}{\rho^{p}} \frac{\partial p}{\partial y}+\frac{\tau}{\rho^{p}}\left(\frac{\partial \tau_{y x}}{\partial x}+\frac{\partial \tau_{y y}}{\partial y}+\frac{\partial \tau_{y z}}{\partial z}\right), \\
\tilde{w}=w^{p}-\frac{\tau}{\rho^{p}} \frac{\partial p}{\partial z}+\frac{\tau}{\rho^{p}}\left(\frac{\partial \tau_{z x}}{\partial x}+\frac{\partial \tau_{z y}}{\partial y}+\frac{\partial \tau_{z z}}{\partial z}\right), \\
\tilde{E}=E^{p}-\frac{\tau}{\rho^{p}}\left(\frac{\partial p u}{\partial x}+\frac{\partial p v}{\partial y}+\frac{\partial p w}{\partial z}\right)+\frac{\tau}{\rho^{p}}\left(\frac{\partial T_{\tau, 5}^{x}}{\partial x}+\frac{\partial T_{\tau, 5}^{y}}{\partial y}+\frac{\partial T_{\tau, 5}^{z}}{\partial z}\right),
\end{gathered}
$$

где

$$
T_{\tau, 5}^{x}=u \tau_{x x}+v \tau_{y x}+w \tau_{z x}, T_{\tau, 5}^{y}=u \tau_{x y}+v \tau_{y y}+w \tau_{y z}, T_{\tau, 5}^{z}=u \tau_{x z}+v \tau_{y z}+w \tau_{z z}
$$

Давление и компоненты тензора вязких напряжений рассчитываются на нижнем временном слое " $p "$. Заметим, что на этапе "предиктор" плотность не изменяется, т.е. полагается, что значения искомых функций (9) изменяются только за счет действия "силовых" факторов. Таким образом трактуется этот первый шаг в методе крупных частиц [21].

На этапе "корректора" сначала рассчитывается новое значение плотности

$$
\rho^{p+1}=\rho^{p}-\tau\left(\frac{\partial \rho^{p} \tilde{u}}{\partial x}+\frac{\partial \rho^{p} \tilde{v}}{\partial x}+\frac{\partial \rho^{p} \tilde{w}}{\partial x}\right)
$$

а затем - остальных функций

или

$$
\begin{gathered}
\mathbf{U}^{p+1}=\tilde{\mathbf{U}}-\tau\left(\frac{\partial F_{\rho \tilde{V}}^{x}}{\partial x}+\frac{\partial F_{\rho \tilde{V}}^{y}}{\partial y}+\frac{\partial F_{\rho \tilde{V}}^{z}}{\partial z}\right) \\
u^{p+1}=\frac{\rho^{p}}{\rho^{p+1}} \tilde{u}-\frac{\tau}{\rho^{p+1}}\left(\frac{\partial \rho^{p} \tilde{u} \tilde{u}}{\partial x}+\frac{\partial \rho^{p} \tilde{u} \tilde{v}}{\partial y}+\frac{\partial \rho^{p} \tilde{u} \tilde{w}}{\partial z}\right) \\
\tilde{v}^{p+1}=\frac{\rho^{p}}{\rho^{p+1}} \tilde{v}-\frac{\tau}{\rho^{p+1}}\left(\frac{\partial \rho^{p} \tilde{v} \tilde{u}}{\partial x}+\frac{\partial \rho^{p} \tilde{v} \tilde{v}}{\partial y}+\frac{\partial \rho^{p} \tilde{v} \tilde{w}}{\partial z}\right), \\
\tilde{w}^{p+1}=\frac{\rho^{p}}{\rho^{p+1}} \tilde{w}-\frac{\tau}{\rho^{p+1}}\left(\frac{\partial \rho^{p} \tilde{w} \tilde{u}}{\partial x}+\frac{\partial \rho^{p} \tilde{w} \tilde{v}}{\partial y}+\frac{\partial \rho^{p} \tilde{w} \tilde{w}}{\partial z}\right), \\
E^{p+1}=\frac{\rho^{p}}{\rho^{p+1}} \tilde{E}-\frac{\tau}{\rho^{p+1}}\left(\frac{\partial \rho^{p} \tilde{E} \tilde{u}}{\partial x}+\frac{\partial \rho^{p} \tilde{E} \tilde{v}}{\partial y}+\frac{\partial \rho^{p} \tilde{E} \tilde{w}}{\partial z}\right)
\end{gathered}
$$




\section{3. Формулировка 3D конечно-объемного метода для тетраэдральной сетки}

В данном разделе будут представлены расчетные соотношения для конечно-объемного представления функций и их производных применительно к тетраэдральному элементарному объему. Расчетные соотношения метода конечного объема получаются интегрированием (1) по объему выделенной в пространстве элементарной расчетной ячейки (в нашем случае - тетраэдра).

Напомним, что по теореме Гаусса-Остроградского для произвольной векторной функции А

$$
\begin{aligned}
\int_{W_{L}} \operatorname{div} \mathbf{A d} W=\int_{W_{L}}\left(\frac{\partial A_{x}}{\partial x}+\frac{\partial A_{y}}{\partial y}+\frac{\partial A_{z}}{\partial z}\right) \mathrm{d} W=\oint_{S_{L}} \mathbf{A d} \mathbf{S} & =\oint_{S_{L}}\left(A_{x} \mathbf{i}+A_{y} \mathbf{j}+A_{z} \mathbf{k}\right)\left(\mathbf{i} \mathrm{d} S_{x}+\mathbf{j} \mathrm{d} S_{y}+\mathbf{k} \mathrm{d} S_{z}\right)= \\
& =\oint_{S_{L}} A_{x} \mathrm{~d} S_{x}+\oint_{S_{L}} A_{y} \mathrm{~d} S_{y}+\oint_{S_{L}} A_{z} \mathrm{~d} S_{z},
\end{aligned}
$$

где $W_{L}, S_{L}$ - объем и поверхность выделенной ячейки $L ; \mathbf{i}, \mathbf{j}, \mathbf{k}$ - единичные орты прямоугольной декартовой системы координат.

В силу независимости функций $A_{x}, A_{y}, A_{z}$ можем положить

$$
\int_{W_{L}}\left(\frac{\partial A_{x}}{\partial x}\right) \mathrm{d} W=\oint_{S} A_{x} \mathrm{~d} S_{x}, \int_{W_{L}}\left(\frac{\partial A_{x}}{\partial y}\right) \mathrm{d} W=\oint_{S} A_{y} \mathrm{~d} S_{y}, \int_{W_{L}}\left(\frac{\partial A_{z}}{\partial z}\right) \mathrm{d} W=\oint_{S} A_{z} \mathrm{~d} S_{z},
$$

где $W_{L}, S_{L}$ - объем и поверхность выделенной ячейки $L$.

Отсюда следует способ расчета усредненных по объему производных

$$
\left\langle\frac{\partial f}{\partial x}\right\rangle_{W_{L}}=\frac{1}{W_{L}} \int_{W_{L}} \frac{\partial f}{\partial x} \mathrm{~d} W=\frac{1}{W_{L}} \oint_{S_{L}} f \mathbf{i} \mathrm{d} \mathbf{S} \approx \frac{1}{W_{L}} \sum_{j=1}^{4} f_{L} S_{L_{j}} \omega_{x}^{L_{j}} \approx \frac{1}{W_{L}} \sum_{j=1}^{4} \frac{1}{2}\left(f_{L}+f_{L B}\right) S_{L_{j}} \omega_{x}^{L_{j}}
$$

Здесь использованы $\mathrm{d} S_{x}, \mathrm{~d} S_{y}, \mathrm{~d} S_{z}$ - проекции элементарной площади $\mathrm{d} S$ на оси $x, y, z$. Они определяются по формулам

$$
\mathrm{d} S_{x}=\omega_{n, x} \mathrm{~d} S, \mathrm{~d} S_{y}=\omega_{n, y} \mathrm{~d} S, \mathrm{~d} S_{z}=\omega_{n, z} \mathrm{~d} S,
$$

где $\omega_{n, x}, \omega_{n, y}, \omega_{n, z}-$ направляющие косинусы внешней нормали к элементу поверхности $j$ по отношению к координатным осям $x, y, z$.

Интегрирование по поверхности $S_{L}$ элементарного объема $L$ проводится для функций $f$ вдоль граней объема $j$ (площадью $S_{L_{j}}$ ), поэтому использование полусуммы значений вблизи данной $(L)$ и соседней ячейки $\left(L B_{j}\right)$ несколько повышает точность расчета.

По аналогии

$$
\begin{aligned}
& \left\langle\frac{\partial f}{\partial y}\right\rangle_{W_{L}}=\frac{1}{W_{L}} \int_{W_{L}} \frac{\partial f}{\partial y} \mathrm{~d} W=\frac{1}{W_{L}} \oint_{S_{L}} f \mathbf{j} \mathrm{d} \mathbf{S} \approx \frac{1}{W_{L}} \sum_{j=1}^{4} f_{L} S_{L_{j}} \omega_{y}^{L_{j}} \approx \frac{1}{W_{L}} \sum_{j=1}^{4} \frac{1}{2}\left(f_{L}+f_{L B_{j}}\right) S_{L_{j}} \omega_{y}^{L_{j}}, \\
& \left\langle\frac{\partial f}{\partial z}\right\rangle_{W_{L}}=\frac{1}{W_{L}} \int_{W_{L}} \frac{\partial f}{\partial z} \mathrm{~d} W=\frac{1}{W_{L}} \oint_{S_{L}} f \mathbf{k} \mathrm{d} \mathbf{S} \approx \frac{1}{W_{L}} \sum_{j=1}^{4} f_{L} S_{L_{j}} \omega_{z}^{L_{j}} \approx \frac{1}{W_{L}} \sum_{j=1}^{4} \frac{1}{2}\left(f_{L}+f_{L B_{j}}\right) S_{L_{j}} \omega_{z}^{L_{j}}
\end{aligned}
$$

Аналогично определяются усредненные по объему $W_{L}$ производные компонент тензора вязких напряжений 


$$
\begin{aligned}
& \left\langle\frac{\partial \tau_{p q}}{\partial x}\right\rangle_{W_{L}} \approx \frac{1}{W_{L}} \sum_{j=1}^{4} \frac{1}{2}\left(\left\langle\tau_{p q}^{L}\right\rangle+\left\langle\tau_{p q}^{L B_{j}}\right\rangle\right) S_{L_{j}} \omega_{x}^{L_{j}}, \\
& \left\langle\frac{\partial \tau_{p q}}{\partial y}\right\rangle_{W_{L}} \approx \frac{1}{W_{L}} \sum_{j=1}^{4} \frac{1}{2}\left(\left\langle\tau_{p q}^{L}\right\rangle+\left\langle\tau_{p q}^{L B_{j}}\right\rangle\right) S_{L_{j}} \omega_{y}^{L_{j}}, \\
& \left\langle\frac{\partial \tau_{p q}}{\partial z}\right\rangle_{W_{L}} \approx \frac{1}{W_{L}} \sum_{j=1}^{4} \frac{1}{2}\left(\left\langle\tau_{p q}^{L}\right\rangle+\left\langle\tau_{p q}^{L B_{j}}\right\rangle\right) S_{L_{j}} \omega_{z}^{L_{j}}
\end{aligned}
$$

Усредненные компоненты тензора вязких напряжений рассчитываются с использованием выражений для усредненных значений производных компонент скорости

$$
\begin{gathered}
\left\langle\tau_{x x}^{L}\right\rangle \approx \frac{2}{3} \mu_{L}\left[2\left\langle\frac{\partial u}{\partial x}\right\rangle_{L}-\left(\left\langle\frac{\partial v}{\partial y}\right\rangle_{L}+\left\langle\frac{\partial w}{\partial z}\right\rangle_{L}\right)\right], \\
\left\langle\tau_{y y}^{L}\right\rangle \approx \frac{2}{3} \mu_{L}\left[2\left\langle\frac{\partial v}{\partial y}\right\rangle_{L}-\left(\left\langle\frac{\partial u}{\partial x}\right\rangle_{L}+\left\langle\frac{\partial w}{\partial z}\right\rangle_{L}\right)\right], \\
\left\langle\tau_{z z}^{L}\right\rangle \approx \frac{2}{3} \mu_{L}\left[2\left\langle\frac{\partial w}{\partial z}\right\rangle_{L}-\left(\left\langle\frac{\partial u}{\partial x}\right\rangle_{L}+\left\langle\frac{\partial v}{\partial y}\right\rangle_{L}\right)\right], \\
\left\langle\tau_{x y}^{L}\right\rangle=\left\langle\tau_{y x}^{L}\right\rangle \approx \mu_{L}\left[\left\langle\frac{\partial u}{\partial y}\right\rangle_{L}+\left\langle\frac{\partial v}{\partial x}\right\rangle_{L}\right], \\
\left\langle\tau_{x z}^{L}\right\rangle=\left\langle\tau_{z x}^{L}\right\rangle \approx \mu_{L}\left[\left\langle\frac{\partial w}{\partial x}\right\rangle_{L}+\left\langle\frac{\partial u}{\partial z}\right\rangle_{L}\right], \\
\left\langle\tau_{y z}^{L}\right\rangle=\left\langle\tau_{z y}^{L}\right\rangle \approx \mu_{L}\left[\left\langle\frac{\partial v}{\partial z}\right\rangle_{L}+\left\langle\frac{\partial w}{\partial y}\right\rangle_{L}\right]
\end{gathered}
$$

Для завершения вычислений осталось определить средние производные компонент скорости

$$
\left\langle\frac{\partial V_{p}}{\partial x_{q}}\right\rangle_{W_{L}} \approx \frac{1}{W_{L}} \sum_{j=1}^{4} \frac{1}{2}\left(V_{p, L}+V_{p, L B_{j}}\right) S_{L_{j}} \omega_{q}^{L_{j}}
$$

где $V_{p}=u, v, w ; p, q=x, y, z$.

\section{4. Численный метод интегрирования конечно-объемных уравнений}

Получим расчетные соотношения для определения функций $\tilde{u}, \tilde{v}, \tilde{w}, \tilde{E}$. С целью нахождения промежуточного значения $\tilde{u}$ в элементарном тетраэдре с номером $L$ выберем 2-ю строку в векторе функции $\mathbf{U}$ и запишем для нее скалярное уравнение

$$
\rho_{L}^{p} \frac{\tilde{u}_{L}-u_{L}^{p}}{\tau}=-\frac{\partial p}{\partial x}+\frac{\partial \tau_{x x}}{\partial x}+\frac{\partial \tau_{x y}}{\partial y}+\frac{\partial \tau_{x z}}{\partial z}
$$

Интегрируя по объему $W_{L}$, получаем 


$$
W_{L} \rho_{L}^{p} \frac{\tilde{u}_{L}-u_{L}^{p}}{\tau}=-\int_{W_{L}} \frac{\partial p}{\partial x} \mathrm{~d} W+\int_{W_{L}} \frac{\partial \tau_{x x}}{\partial x} \mathrm{~d} W+\int_{W_{L}} \frac{\partial \tau_{x y}}{\partial y} \mathrm{~d} W+\int_{W_{L}} \frac{\partial \tau_{x z}}{\partial z} \mathrm{~d} W
$$

Выше были определены формулы интегрирования по элементарному тетраэдральному объему, в соответствии с которыми

$$
\begin{aligned}
& \tilde{u}_{L}=u_{L}^{p}-\frac{\tau}{\rho^{p} W_{L}}\left[\sum_{j=1}^{4} S_{L_{j}} \omega_{x}^{L_{j}} \frac{1}{2}\left(p_{L}+p_{L B_{j}}\right)\right]+ \\
&+\frac{\tau}{\rho^{p} W_{L}}\left[\sum_{j=1}^{4} S_{L_{j}} \omega_{x}^{L_{j}} \frac{1}{2}\left(\left\langle\tau_{x x}^{L}\right\rangle+\left\langle\tau_{x x}^{L B_{j}}\right\rangle\right)+S_{L_{j}} \omega_{y}^{L_{j}} \frac{1}{2}\left(\left\langle\tau_{x y}^{L}\right\rangle+\left\langle\tau_{x y}^{L B_{j}}\right\rangle\right)+\right. \\
&\left.+S_{L_{j}} \omega_{z}^{L_{j}} \frac{1}{2}\left(\left\langle\tau_{x z}^{L}\right\rangle+\left\langle\tau_{x z}^{L B_{j}}\right\rangle\right)\right],
\end{aligned}
$$

где $\omega_{x}^{L_{j}}, \omega_{y}^{L_{j}}, \omega_{z}^{L_{j}}$ - направляющие косинусы единичной внешней нормали к грани $j$ элемента $L ; S_{L_{j}}$ - площадь $j$-й грани элемента $L ;\left\langle\tau_{x x}^{L}\right\rangle,\left\langle\tau_{x x}^{L B_{j}}\right\rangle,\left\langle\tau_{x y}^{L}\right\rangle,\left\langle\tau_{x y}^{L B_{j}}\right\rangle,\left\langle\tau_{x z}^{L}\right\rangle,\left\langle\tau_{x z}^{L B_{j}}\right\rangle$ - усредненные значения компонент тензора напряжений в элементе $L$ и в элементе $L B_{j}$, граничащим с элементом $L$ через грань $L_{j}$.

По аналогии

$$
\begin{aligned}
& \tilde{v}_{L}=v_{L}^{p}-\frac{\tau}{\rho^{p} W_{L}}\left[\sum_{j=1}^{4} S_{L_{j}} \omega_{y}^{L_{j}} \frac{1}{2}\left(p_{L}+p_{L B_{j}}\right)\right]+ \\
& +\frac{\tau}{\rho^{p} W_{L}}\left[\sum_{j=1}^{4} S_{L_{j}} \omega_{x}^{L_{j}} \frac{1}{2}\left(\left\langle\tau_{x y}^{L}\right\rangle+\left\langle\tau_{x y}^{L B_{j}}\right\rangle\right)+S_{L_{j}} \omega_{y}^{L_{j}} \frac{1}{2}\left(\left\langle\tau_{y y}^{L}\right\rangle+\left\langle\tau_{y y}^{L B_{j}}\right\rangle\right)+\right. \\
& \left.+S_{L_{j}} \omega_{z}^{L_{j}} \frac{1}{2}\left(\left\langle\tau_{y z}^{L}\right\rangle+\left\langle\tau_{y z}^{L B_{j}}\right\rangle\right)\right], \\
& \tilde{w}_{L}=w_{L}^{p}-\frac{\tau}{\rho^{p} W_{L}}\left[\sum_{j=1}^{4} S_{L_{j}} \omega_{z}^{L_{j}} \frac{1}{2}\left(p_{L}+p_{L B_{j}}\right)\right]+ \\
& +\frac{\tau}{\rho^{p} W_{L}}\left[\sum_{j=1}^{4} S_{L_{j}} \omega_{x}^{L_{j}} \frac{1}{2}\left(\left\langle\tau_{x z}^{L}\right\rangle+\left\langle\tau_{x z}^{L B_{j}}\right\rangle\right)+S_{L_{j}} \omega_{y}^{L_{j}} \frac{1}{2}\left(\left\langle\tau_{y z}^{L}\right\rangle+\left\langle\tau_{y z}^{L B_{j}}\right\rangle\right)+\right. \\
& \left.+S_{L_{j}} \omega_{z}^{L_{j}} \frac{1}{2}\left(\left\langle\tau_{z z}^{L}\right\rangle+\left\langle\tau_{z z}^{L B_{j}}\right\rangle\right)\right], \\
& \tilde{E}=E^{p}-\frac{\tau}{\rho^{p} W_{L}}\left[\sum_{j=1}^{4} S_{L_{j}} \omega_{x}^{L_{j}} \frac{1}{2}\left(p_{L} u_{L}+p_{L B_{j}} u_{L B_{j}}\right)+S_{L_{j}} \omega_{x}^{L_{j}} \frac{1}{2}\left(p_{L} v_{L}+p_{L B_{j}} v_{L B_{j}}\right)\right]+ \\
& +\frac{\tau}{\rho^{p} W_{L}}\left[\sum_{j=1}^{4} S_{L_{j}} \omega_{x}^{L_{j}} \frac{1}{2}\left(T_{\tau, 5, L_{j}}^{x}+T_{\tau, 5, L B_{j}}^{x}\right)+S_{L_{j}} \omega_{y}^{L_{j}} \frac{1}{2}\left(T_{\tau, 5, L_{j}}^{y}+T_{\tau, 5, L B_{j}}^{y}\right)+\right. \\
& \left.+S_{L_{j}} \omega_{z}^{L_{j}} \frac{1}{2}\left(T_{\tau, 5, L_{j}}^{z}+T_{\tau, 5, L B_{j}}^{z}\right)\right],
\end{aligned}
$$

где функции $T_{\tau, 5, L_{j}}^{p}$ рассчитываются по формулам

$$
T_{\tau, 5, L_{j}}^{x}=\tilde{u}_{L}\left\langle\tau_{x x}^{L}\right\rangle+\tilde{v}_{L}\left\langle\tau_{y x}^{L}\right\rangle+\tilde{w}_{L}\left\langle\tau_{z x}^{L}\right\rangle,
$$




$$
\begin{aligned}
& T_{\tau, 5, L_{j}}^{y}=\tilde{u}_{L}\left\langle\tau_{x y}^{L}\right\rangle+\tilde{v}_{L}\left\langle\tau_{y y}^{L}\right\rangle+\tilde{w}_{L}\left\langle\tau_{y z}^{L}\right\rangle, \\
& T_{\tau, 5, L_{j}}^{z}=\tilde{u}_{L}\left\langle\tau_{x z}^{L}\right\rangle+\tilde{v}_{L}\left\langle\tau_{y z}^{L}\right\rangle+\tilde{w}_{L}\left\langle\tau_{z z}^{L}\right\rangle
\end{aligned}
$$

После определения промежуточных значений компонент скорости можно обратиться к предварительному решению уравнения неразрывности

или

$$
\int_{W_{L}} \frac{\partial \rho}{\partial t} \mathrm{~d} W+\int_{W_{L}} \operatorname{div}(\rho \tilde{\mathbf{V}}) \mathrm{d} W=0
$$

или

$$
\begin{aligned}
& \frac{\rho_{L}^{p+1}-\rho_{L}^{p}}{\tau} W_{L}+\oint_{S_{L}} \rho \tilde{\mathbf{V}}_{L} \mathrm{~d} \mathbf{S}=0 \\
& \frac{\rho_{L}^{p+1}-\rho_{L}^{p}}{\tau} W_{L}+\sum_{j=1}^{4} S_{L_{j}} G_{L_{j}}=0
\end{aligned}
$$

Если $\boldsymbol{\Omega}_{S_{L}}$ - единичный вектор внешней нормали к элементу поверхности $\mathrm{d} S$, то при положительной величине плотности потока через элемент поверхности $\mathrm{d} S$

$$
G_{L, \mathrm{~d} S}=\rho\left(\tilde{\mathbf{V}}_{S_{L}} \cdot \boldsymbol{\Omega}_{S_{L}}\right)>0
$$

газ из объема вытекает, а при $G_{L, \mathrm{~d} S}<0$ газ втекает в объем.

При конечно-разностном представлении

$$
\begin{gathered}
\rho_{L}^{p+1}=\rho_{L}^{p}-\frac{\tau}{W_{L}} \sum_{j=1}^{4} S_{L_{j}} \rho_{L_{j}} \tilde{V}_{L_{L_{j}}}, \\
\rho_{L_{j}}=\left\{\begin{array}{l}
\rho_{L}^{p}, \tilde{V}_{S_{L_{j}}}>0 \\
\rho_{L B_{j}}^{p}, \tilde{V}_{S_{L_{j}}}<0
\end{array}\right.
\end{gathered}
$$

$\tilde{V}_{S_{L_{j}}}-$ нормальная скорость к грани $j$.

Расчет величины $\tilde{V}_{L_{j}}$ требует использования некоторых дополнительных соображений. Один из простейших способов состоит в следующем. Учитывая, что на шаге предиктора были найдены усредненные по элементарным объемам компоненты скорости $\tilde{u}_{L}, \tilde{v}_{L}, \tilde{w}_{L}$, с их использованием находится искомая нормальная компонента скорости

$$
\tilde{V}_{S_{L_{j}}}=\tilde{u}_{L} \omega_{S_{L_{j}}}^{x}+\tilde{v}_{L} \omega_{S_{L_{j}}}^{y}+\tilde{w}_{L} \omega_{S_{L_{j}}}^{z}
$$

По аналогии, для нормальной компоненты скорости на той же самой грани соседнего объема

$$
\tilde{V}_{L B_{j}}=-\tilde{u}_{L B_{j}} \omega_{S_{L_{j}}}^{x}-\tilde{v}_{L B_{j}} \omega_{S_{L_{j}}}^{y}-\tilde{w}_{L B_{j}} \omega_{S_{L_{j}}}^{z}
$$

Заметим, что направляющие косинусы в данном случае по-прежнему определяют внешнюю нормаль к граням объема $L$.

Для удобства вычислений вводим две вспомогательных величины

$$
\tilde{V}_{S_{L_{j}}^{+}}^{+}=\frac{1}{2}\left(\tilde{V}_{S_{L_{j}}}+\left|\tilde{V}_{S_{L_{j}}}\right|\right), \quad \tilde{V}_{S_{L_{j}}}^{-}=\frac{1}{2}\left(\tilde{V}_{S_{L_{j}}}-\left|\tilde{V}_{S_{L_{j}}}\right|\right)
$$


Тогда соотношение(18) может быть записано в виде

$$
\rho_{L}^{p+1}=\rho_{L}^{p}-\frac{\tau}{W_{L}} \sum_{j=1}^{4} S_{L_{j}}\left(\rho_{L_{j}} \tilde{V}_{S_{L_{j}}}^{+}+\rho_{L B_{j}} \tilde{V}_{S_{L_{j}}^{-}}\right)
$$

По этой же схеме производится расчет компонент скорости и полной энергии на заключительном этапе корректора

$$
\begin{aligned}
& u_{L}^{p+1}=\tau \frac{\rho_{L}^{p}}{\rho_{L}^{p+1}} \tilde{u}_{L}-\frac{\tau}{\rho_{L}^{p+1}} \sum_{j=1}^{4} S_{L_{j}}\left(\rho_{L_{j}} \tilde{u}_{L} \tilde{V}_{S_{L_{j}}}^{+}+\rho_{L B_{j}} \tilde{u}_{L B_{j}} \tilde{V}_{S_{L_{j}}}^{-}\right), \\
& v_{L}^{p+1}=\tau \frac{\rho_{L}^{p}}{\rho_{L}^{p+1}} \tilde{v}_{L}-\frac{\tau}{\rho_{L}^{p+1}} \sum_{j=1}^{4} S_{L_{j}}\left(\rho_{L_{j}} \tilde{v}_{L} \tilde{V}_{S_{L_{j}}}^{+}+\rho_{L B_{j}} \tilde{v}_{L B_{j}} \tilde{V}_{S_{L_{j}}}^{-}\right), \\
& E_{L}^{p+1}=\tau \frac{\rho_{L}^{p}}{\rho_{L}^{p+1}} \tilde{E}_{L}-\frac{\tau}{\rho_{L}^{p+1}} \sum_{j=1}^{4} S_{L_{j}}\left(\rho_{L_{j}} \tilde{V}_{S_{L_{j}}}^{+} \tilde{E}_{L}+\rho_{L B_{j}} \tilde{V}_{S_{L_{j}}}^{-} \tilde{E}_{L B_{j}}\right)
\end{aligned}
$$

Для численного интегрирования системы уравнений движения газа сформулируем граничные условия.

1. Граничные условия непротекания (для уравнений Эйлера).

Для каждого тетраэдрального элемента, примыкающего одной из граней обтекаемой поверхности можно определить локальную внешнюю нормаль к указанной грани. Направляющие косинусы этой нормали $\omega_{L B}^{x}, \omega_{L B}^{y}, \omega_{L B}^{z}$ уже определены в сеточном препроцессоре, поэтому нормальная компонента скорости к указанной грани определяется по формуле

$$
V_{n, L B}=u_{L} \omega_{L B}^{x}+v_{L} \omega_{L B}^{y}+w_{L} \omega_{L B}^{z}
$$

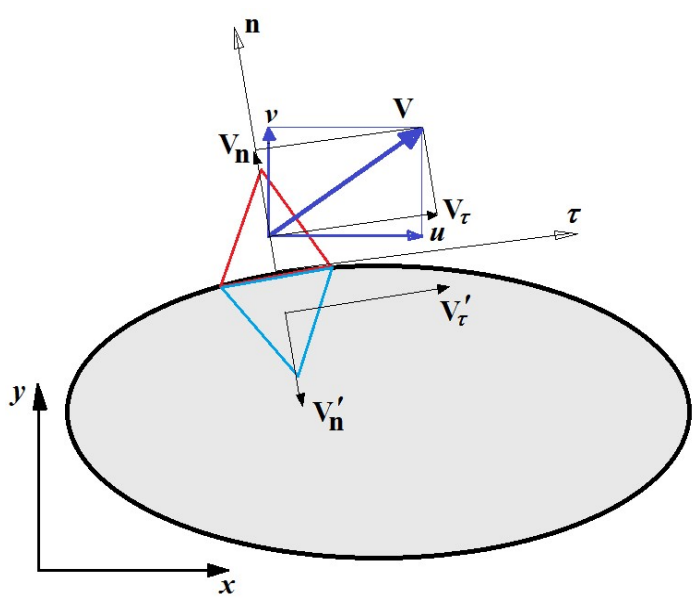

К формулировке граничных условий для неструктурированной тетраэдральной сетки

Условие непротекания обтекаемой поверхности физически эквивалентно тому, что нормальная компонента скорости в фиктивной ячейке равна по модулю и противоположна направлению найденной выше нормальной компоненте скорости. Тогда проекции скорости в фиктивной ячейке должны удовлетворять условию

$$
V_{n, x, L B}^{\prime}=u_{n, L B}^{\prime}=-V_{n, L B} \omega_{L B}^{x}, \quad V_{n, y, L B}^{\prime}=v_{n, L B}^{\prime}=-V_{n, L B} \omega_{L B}^{y}, \quad V_{n, z, L B}^{\prime}=w_{n, L B}^{\prime}=-V_{n, L B} \omega_{L B}^{z}
$$

2. $\mathrm{B}$ случае использования условий прилипания (при решении уравнений Навье-Стокса) все компоненты скорости на поверхности должны равняться нулю, поэтому 


$$
u_{n, L B}^{\prime}=-u_{n, L B}, \quad v_{n, L B}^{\prime}=-v_{n, L B}, \quad w_{n, L B}^{\prime}=-w_{n, L B}
$$

\section{5. Система уравнений и алгоритм численного интегрирования, используемые в компьютерном коде PERAT-3D}

B авторском компьютерном коде PERAT-3D реализована расчетно-теоретическая модель, основанная на системе самосогласованных уравнений вязкого и теплопроводного газа, интегрируемых на структурированных многоблочных сетках. В указанную систему входят уравнения Навье - Стокса и неразрывности, уравнения сохранения энергии и уравнение состояния термически совершенного газа. Математическая формулировка имеет вид

$$
\begin{aligned}
\frac{\partial \mathbf{U}}{\partial t}+\frac{\partial u \mathbf{U}}{\partial x}+\frac{\partial v \mathbf{U}}{\partial y}+\frac{\partial w \mathbf{U}}{\partial z} & =\mathbf{R}, \quad \mathbf{U}=\left(\mathbf{U}_{g}, T\right)^{T}, \quad \mathbf{R}=\left(\mathbf{R}_{g}, \frac{R_{T}}{\rho c_{p}}\right)^{T} ; \\
\mathbf{U}_{g} & =(\rho, \rho u, \rho v, \rho w)^{T},
\end{aligned}
$$

где

$$
\begin{gathered}
\mathbf{R}_{g}=\left(0 ;-\frac{\partial p}{\partial x}+S_{\mu, x} ;-\frac{\partial p}{\partial y}+S_{\mu, y} ;-\frac{\partial p}{\partial z}+S_{\mu, z}\right)^{T}, \\
S_{\mu, x}=-\frac{2}{3} \frac{\partial}{\partial x}(\mu \nabla \mathbf{V})+\frac{\partial}{\partial x}\left[\mu\left(\frac{\partial v}{\partial x}+\frac{\partial u}{\partial y}\right)\right]+\frac{\partial}{\partial z}\left[\mu\left(\frac{\partial w}{\partial x}+\frac{\partial u}{\partial z}\right)\right]+2 \frac{\partial}{\partial x}\left(\mu \frac{\partial u}{\partial x}\right), \\
S_{\mu, y}=-\frac{2}{3} \frac{\partial}{\partial y}(\mu \nabla \mathbf{V})+\frac{\partial}{\partial y}\left[\mu\left(\frac{\partial w}{\partial y}+\frac{\partial v}{\partial z}\right)\right]+\frac{\partial}{\partial x}\left[\mu\left(\frac{\partial u}{\partial y}+\frac{\partial v}{\partial x}\right)\right]+2 \frac{\partial}{\partial y}\left(\mu \frac{\partial v}{\partial y}\right), \\
S_{\mu, z}=-\frac{2}{3} \frac{\partial}{\partial z}(\mu \nabla \mathbf{V})+\frac{\partial}{\partial z}\left[\mu\left(\frac{\partial u}{\partial z}+\frac{\partial w}{\partial x}\right)\right]+\frac{\partial}{\partial y}\left[\mu\left(\frac{\partial v}{\partial z}+\frac{\partial w}{\partial y}\right)\right]+2 \frac{\partial}{\partial z}\left(\mu \frac{\partial w}{\partial z}\right), \\
R_{T}=\nabla(\lambda \nabla T)+\frac{\partial p}{\partial t}+\mathbf{V} \cdot \nabla p+\Phi \mu
\end{gathered}
$$$$
\Phi_{\mu}=\mu\left[2\left(\frac{\partial u}{\partial x}\right)^{2}+2\left(\frac{\partial v}{\partial y}\right)^{2}+2\left(\frac{\partial w}{\partial z}\right)^{2}+\left(\frac{\partial v}{\partial x}+\frac{\partial u}{\partial y}\right)^{2}+\left(\frac{\partial w}{\partial y}+\frac{\partial v}{\partial z}\right)^{2}+\left(\frac{\partial u}{\partial z}+\frac{\partial w}{\partial x}\right)^{2}-\frac{2}{3}\left(\frac{\partial u}{\partial x}+\frac{\partial v}{\partial y}+\frac{\partial w}{\partial z}\right)^{2}\right]
$$

$t$ - время; $x, y, z$ - ортогональные цилиндрические координаты; $u, v, w$ - проекции вектора скорости $\mathbf{V}$ на оси координат; $p, \rho, T$ - давление, плотность и температура поступательного движения частиц; $\mu, \lambda, c_{p}$ - динамический коэффициент вязкости, коэффициент теплопроводности и удельная теплоемкость смеси при постоянном давлении.

Замыкающие соотношения для решаемой системы уравнений включают в себя термическое и калорическое уравнения состояния идеального газа

$$
p=\frac{\rho R_{0} T}{M_{\Sigma}}=(\gamma-1) \rho e, e=c_{V} T,
$$

где $M_{\Sigma}-$ молекулярный вес газа.

В набегающем со скоростью $V_{\infty}$ потоке воздуха задавались параметры атмосферы для заданной высоты полета. На поверхности ЛА задавались условия прилипания. Граничные условия в выходном сечении $x=x_{\max }$ задавались в виде

$$
\frac{\partial \psi}{\partial \xi}=0
$$


где $\psi=\left\{u, v, T, \rho, Y_{i}, e_{V, m}\right\} ; \xi-$ координатная линия, подстраиваемая к линиям тока. На границе с обтекаемым телом задавалась постоянная температура $T_{w}$. Плотность конвективного теплового потока на поверхности с локальной нормалью $\mathbf{n}$ определялась по формуле

$$
q_{c}=-\lambda_{w}(\mathbf{n} \cdot \nabla T)_{w}
$$

Описание численного метода интегрирования системы уравнений (35)-(36), приведена в [14-16].

\section{6. Результаты численного моделирования}

Для проведения численного исследования были выбраны экспериментальные данные [30]. Аэродинамические характеристики двух стандартных гиперзвуковых моделей баллистического типа, обозначенных как HB-1 и НB-2, были исследованы в сверхзвуковом и гиперзвуковом режимах в аэродинамической трубе von Karman Gas Dynamics Facility ARO, Inc.

Испытания проводились при числах Маха 1.5, 2, 3, 4, 5, 8 и 10, в широком диапазоне чисел Рейнольса $\left(0.07\right.$ до $\left.2.55 \times 10^{6}\right)$, в зависимости от диаметра корпуса $(d=3.125,10$ и 19.05 см) и в диапазоне углов атаки от -2 до 15 град. Исследовалось влияние числа Маха, числа Рейнольдса и положения центра приложения сил на статическую устойчивость и осевую силу. Для проведения валидационных численных исследований представляет интерес измерение осевой силы наветренной и подветренной частей моделей, а также измерение нормальной силы при разных углах атаки. Точные размеры испытанных моделей даны на рис. 1

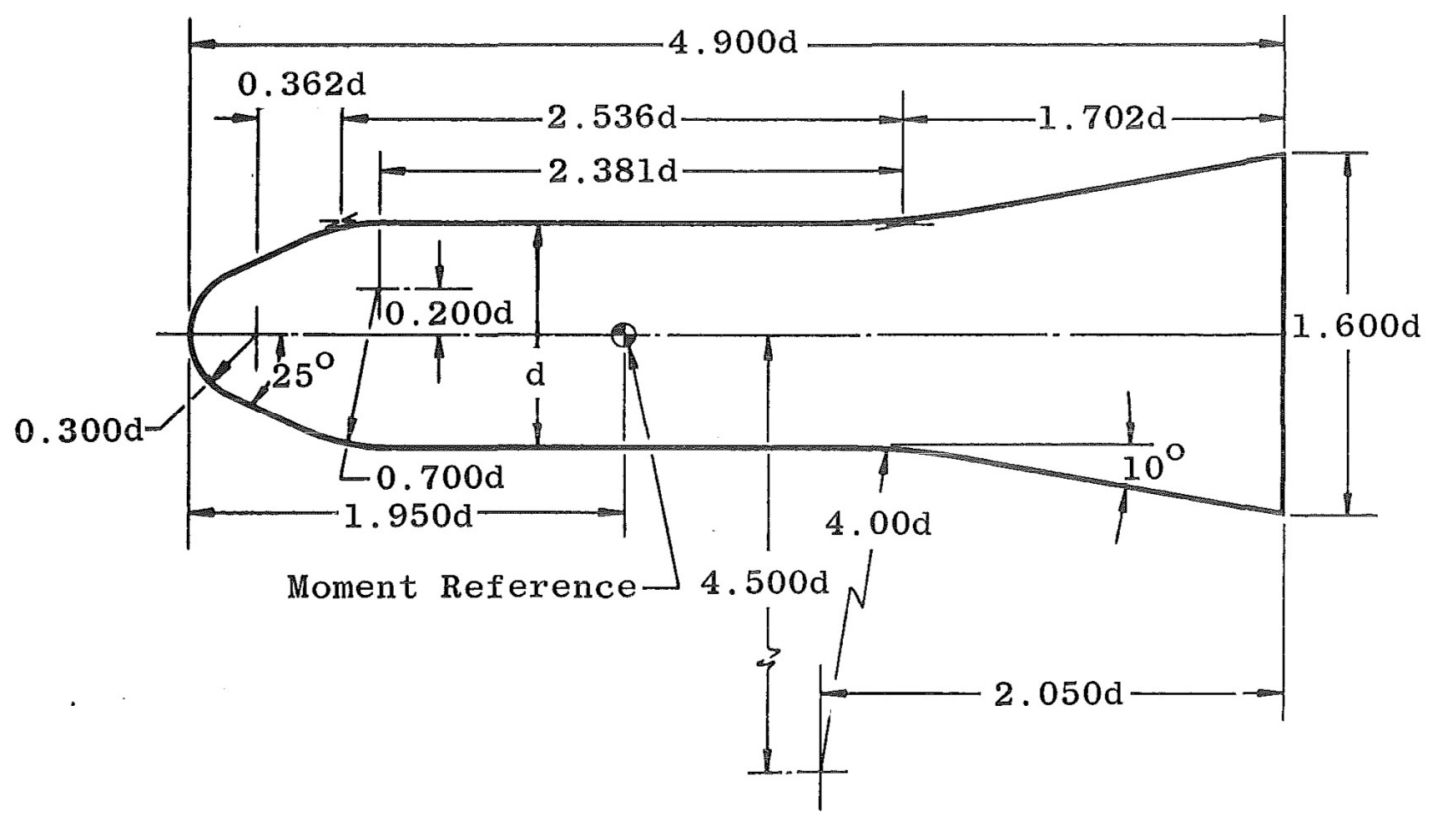

Рис. 1. Геометрия модели НВ-2. В расчетах полагалось $d=10 \mathrm{~cm}$

Часть экспериментальных данных, приведенных в [30], показана на рис. 2 и 3. Эти экспериментальные данные выбраны для сравнения в данной работе.

На рис. 2, $а$ звездочкой показаны результаты расчета коэффициента давления наветренной стороны по коду PERAT-3D. На рис. 2, 6 полым квадратом показана сумма экспериментальных данных коэффициентов сопротивления наветренной и подветренной сторон модели, полым кружком - результаты расчетов по коду USTFEN, а звездочкой - по коду PERAT-3D.

Отметив хорошее совпадение результатов расчетов с экспериментальными данными, рассмотрим основные закономерности течения, которые удается наблюдать по результатам численного моделирования с использованием компьютерных кодов USTFENи PERAT-3D. 


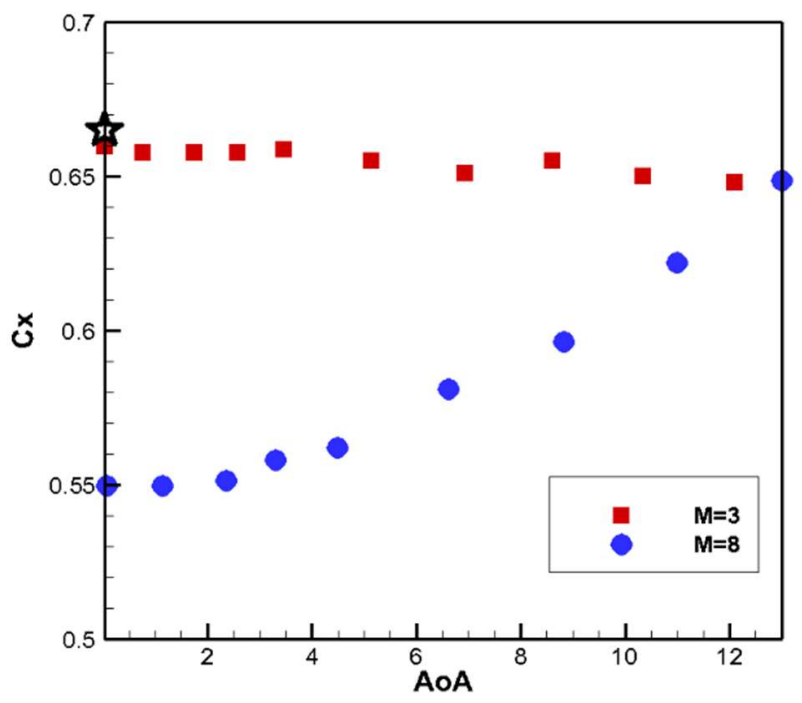

$a$

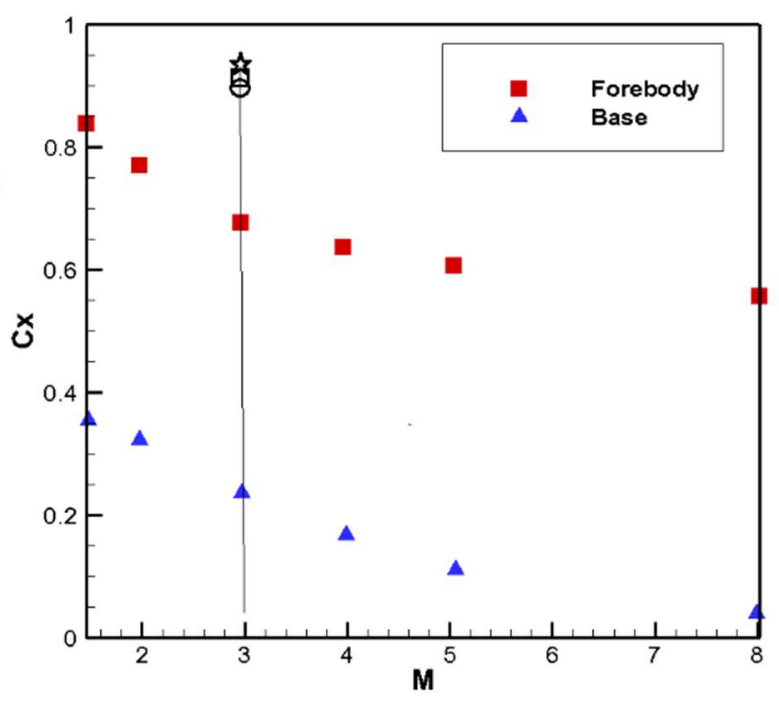

$\sigma$

Рис. 2. Экспериментальные данные испытания модели НВ-2 в аэродинамической трубе. Зависимость осевого коэффициента давления наветренной стороны модели HB-2 от угла атаки для двух чисел Маха $(a)$ и зависимость коэффициента давления подветренной стороны модели для разных чисел Маха при нулевом угле атаки (б)

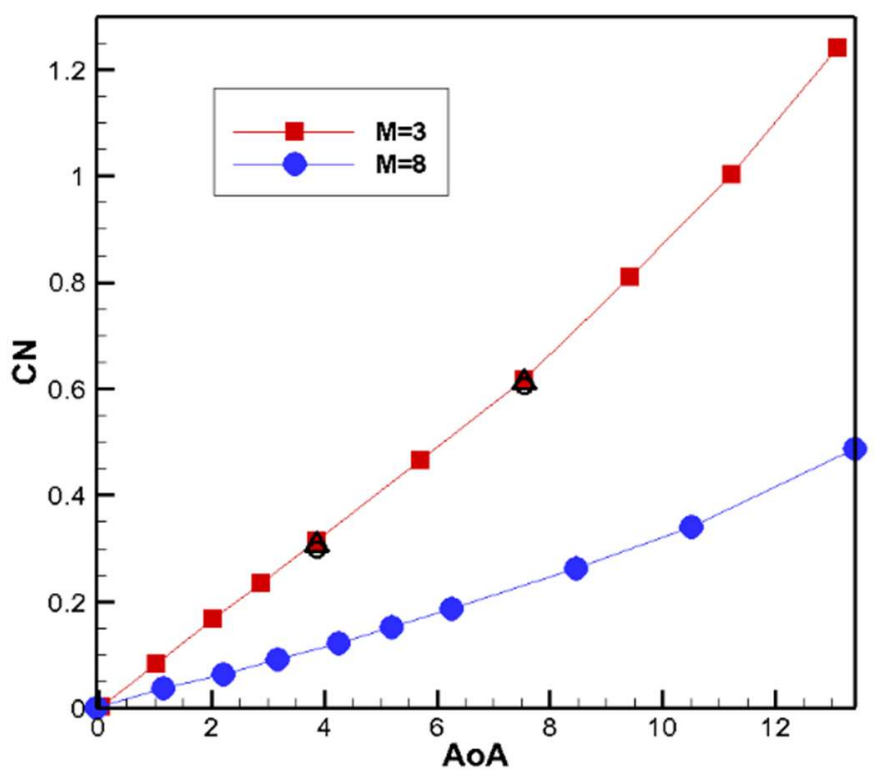

Рис. 3. Экспериментальные данные испытания модели НВ-2 в аэродинамической трубе. Зависимость нормального коэффициента давления от угла атаки для двух чисел Маха. Расчетные данные USTFEN показаны треугольниками, а PERAT-3D - полыми кружками

Численное моделирование аэродинамических характеристик проводилось для исходных данных, представленных в таблице 1.

Таблица N 1

\section{Условия в потоке при проведении численного исследования}

\begin{tabular}{|c|c|c|c|c|c|c|}
\hline$p_{\infty}$, эрг $/ \mathrm{cm}^{3}$ & $\rho_{\infty}, \Gamma / \mathrm{cm}^{3}$ & $T_{\infty}, \mathrm{K}$ & $\gamma$ & $\mathrm{M}_{\infty}$ & $T_{w}, \mathrm{~K}$ & $p_{0}$, эрг $/ \mathrm{cm}^{3}$ \\
\hline 93990 & $0.297 \times 10^{-3}$ & 110.3 & 1.4 & 3 & 250 & $1.17 \times 10^{6}$ \\
\hline
\end{tabular}


Для расчетов по компьютерному коду USTFEN использовалась неструктурированная конечно-объемная сетка, содержащая $N_{\mathrm{pt}}=294935$ узлов, $N_{\mathrm{el}}=1723721$ элементарных объемов и $N_{\text {fict }}=28936$ фиктивных элементарных объемов, расположенных «под поверхностью» обтекаемой модели. Пример расчетной сетки, расчетное распределение плотности и экспериментальная шлирен-фотография [30] при $\mathrm{M}=7$ и $\alpha=2^{0}$ показаны на рис. 4.

B расчетах по компьютерному коду PERAT-3D использовалась многоблочная структурированная конечно-разностная сетка. Геометрия расчетной области и конфигурация отдельных блоков показана на рис. 5. Первые два блока примыкают к области сферического затупления конуса). Внутри каждого блока использовались минимальные расчетные сетки: $\mathrm{NJ}=9 \times \mathrm{NK}=5$ (Блок 1), $\mathrm{NJ}=9 \times \mathrm{NK}=17$ (Блок 2), $\mathrm{NJ}=9 \times \mathrm{NK}=17$ (Блок 3$), \mathrm{NJ}=15 \times \mathrm{NK}=17$ (Блок 4), $\mathrm{NJ}=65 \times \mathrm{NK}=17$ (Блок 5), $\mathrm{NJ}=9 \times \mathrm{NK}=5 \quad$ (Блок 6), $\mathrm{NJ}=13 \times \mathrm{NK}=17 \quad$ (блок 7), $\mathrm{NJ}=57 \times \mathrm{NK}=17$ (Блок 8). Обратим внимание, что топология блоков 1 и 6 отличается от остальных блоков. Сетка в указанных блоках строятся для интегрирования уравнений в окрестности оси симметрии. По нормали к поверхности задавалось NI=57 точек.
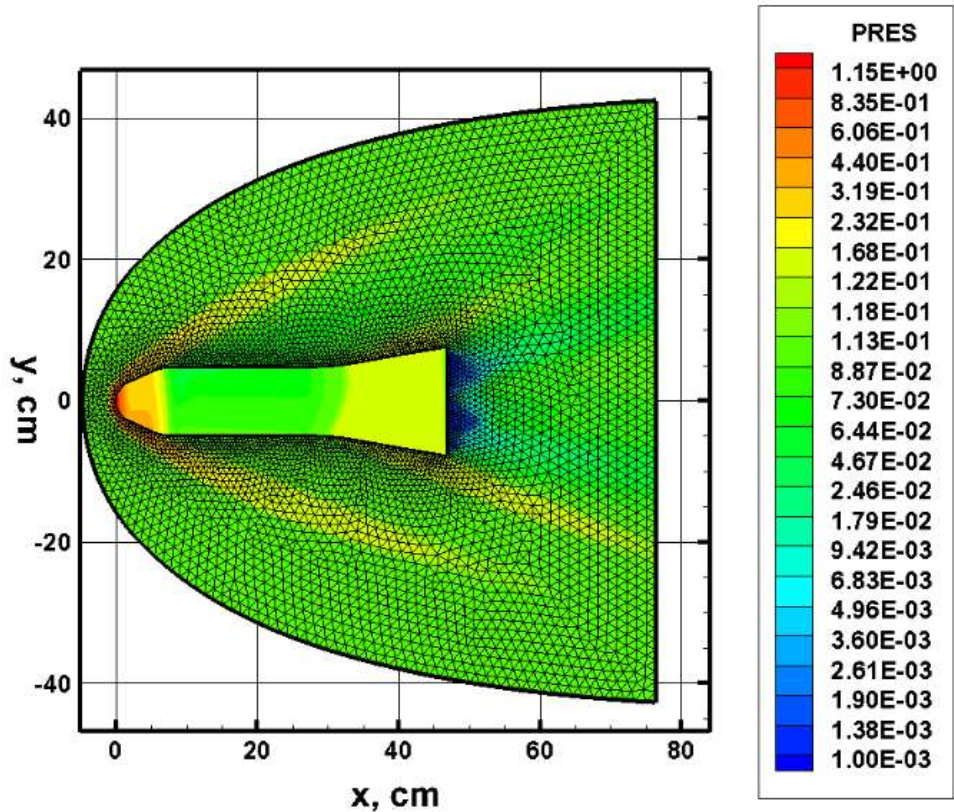

Рис. $4, a$. Неструктурированная расчетная сетка в плоскости $z=0$
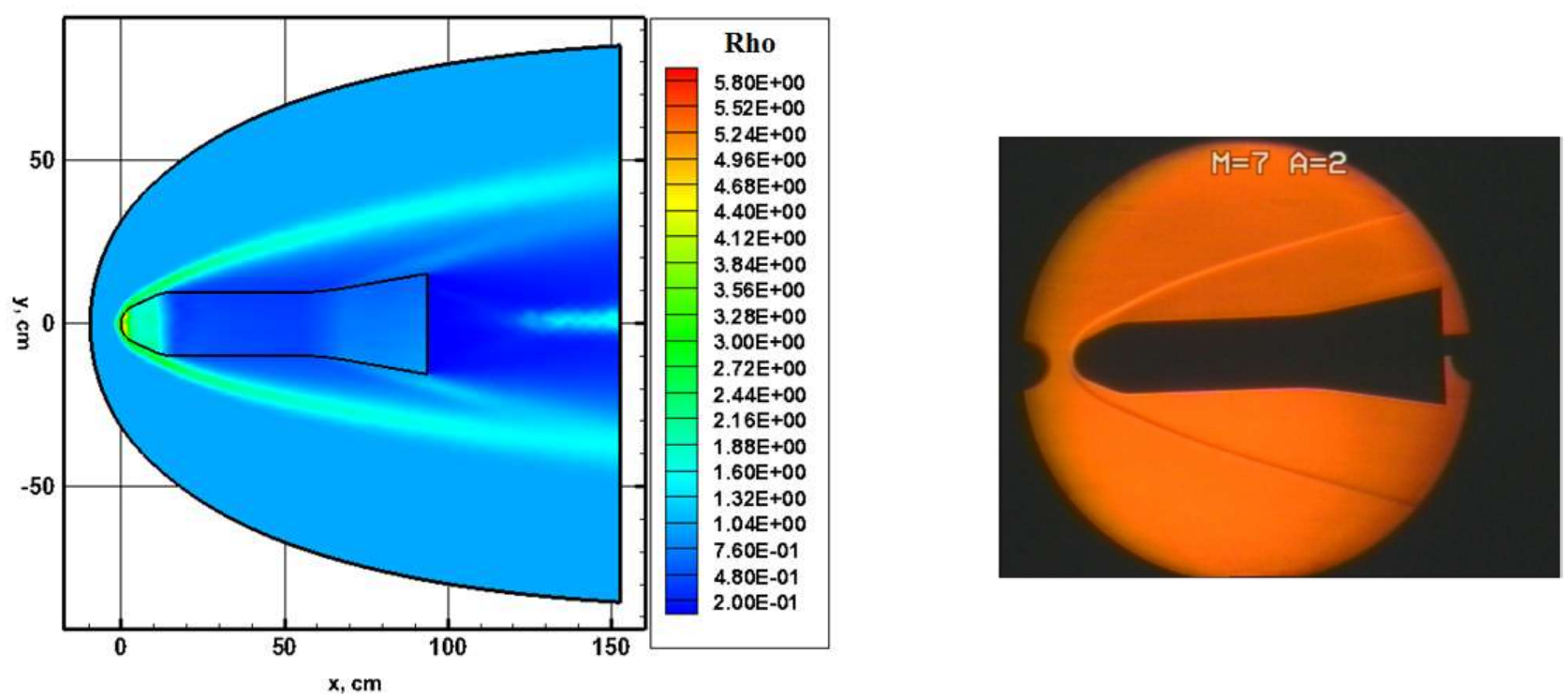

Рис. 4, б. Расчетное распределение плотности и шлирен-фотография модели в аэродинамической трубе 


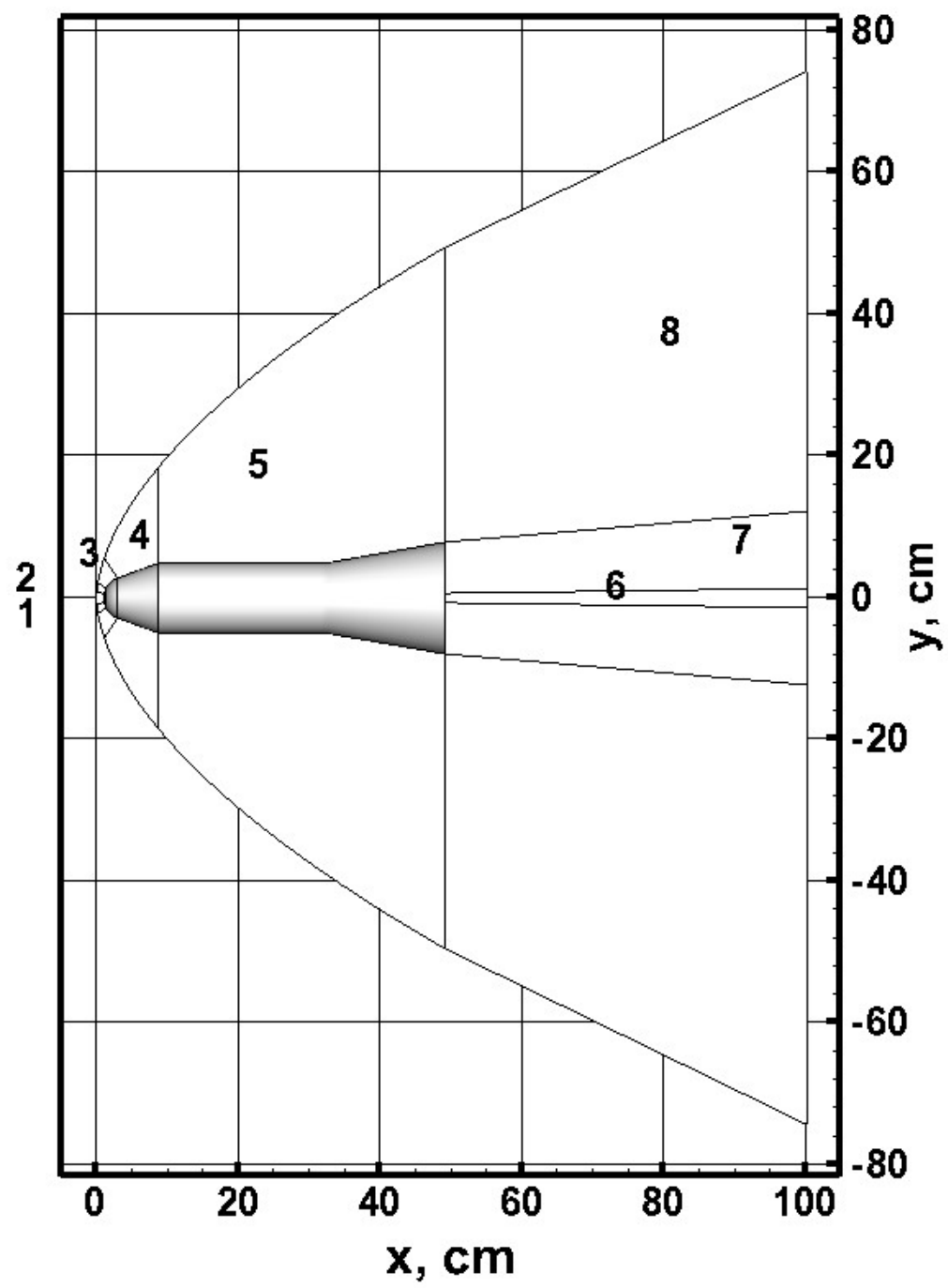

Рис. 5. Многоблочная геометрия расчетной области для кода PERAT-3D

Результаты расчетов с использованием кода USTFEN показаны на рис. 6-11, а с использованием кода PERAT-3D - на рис. 7-13.

Графическое представление расчетных данных по коду USTFEN разбито на три группы по углам атаки $\alpha=0^{\circ}, 4^{\circ}$ и $8^{\circ}$. Для фиксированного угла атаки приведены последовательно поля температуры $(a, \sigma)$, давления $(b, 2)$, чисел Маха $(\partial, e)$ и продольной скорости $(ж, 3)$ в двух проекциях. Распределения плотности и давления в плоскости симметрии $z=0$ показаны на рисунках $(u, \kappa)$. И, наконец, на двух завершающих рисунках каждой серии даны распределения давления и температуры вдоль поверхности в плоскости симметрии $z=0$. Сравнение соответствующих рисунков $(6,7),(8,9)$ и $(10,11)$ позволяет проследить за эволюцией поля течения при увеличении угла атаки.

Используя представленные расчетные данные рассмотрим основные особенности течения, которые можно изучить по расчетным данным кода USTFEN.

На рис. 6, $a, \sigma$ дано поле температур при нулевом угле атаки. Можно выделить шесть характерных областей, которые будут наблюдаться и при других условиях обтекания (см. цифры на поле рис. $6, a$ ). Высокая температура у носовой части (1) отвечает области почти полного торможения набегающего потока. Здесь в очень узкой зоне практически достигается температура торможения ( $300 \mathrm{~K})$. Две зоны повышенной температуры ( 2 и 4$)$ обусловлены сжатием газа отошедшими ударными волнами от передней критической точки и от юбки модели. В области 3 наблюдается пониженная температура, поскольку здесь формируется зона течения разрежения над цилиндрической поверхностью. Наиболее сильное разрежение наблюдается 
за срезом юбки модели (5). Здесь температура падает ниже температуры в набегающем потоке. И, наконец, в 6-й зоне наблюдается заметный нагрев газа. Здесь создается повышенное давление сходящимся к оси симметрии потоком, что также приводит к образованию возвратно-вихревого движения у задней поверхности модели.

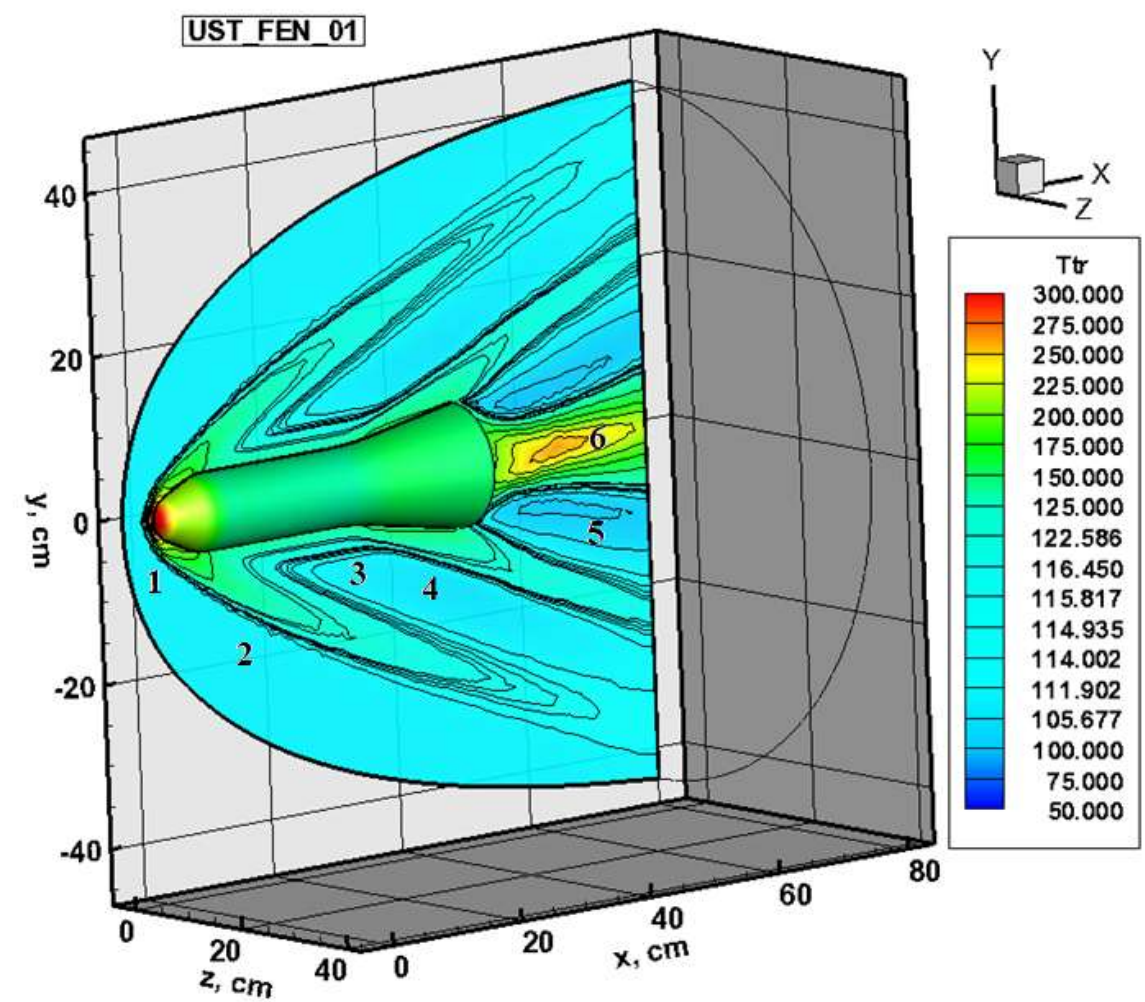

Рис. $6, a$. Поле температуры в К при $\alpha=0$ в плоскости симметрии $\mathrm{z}=0$

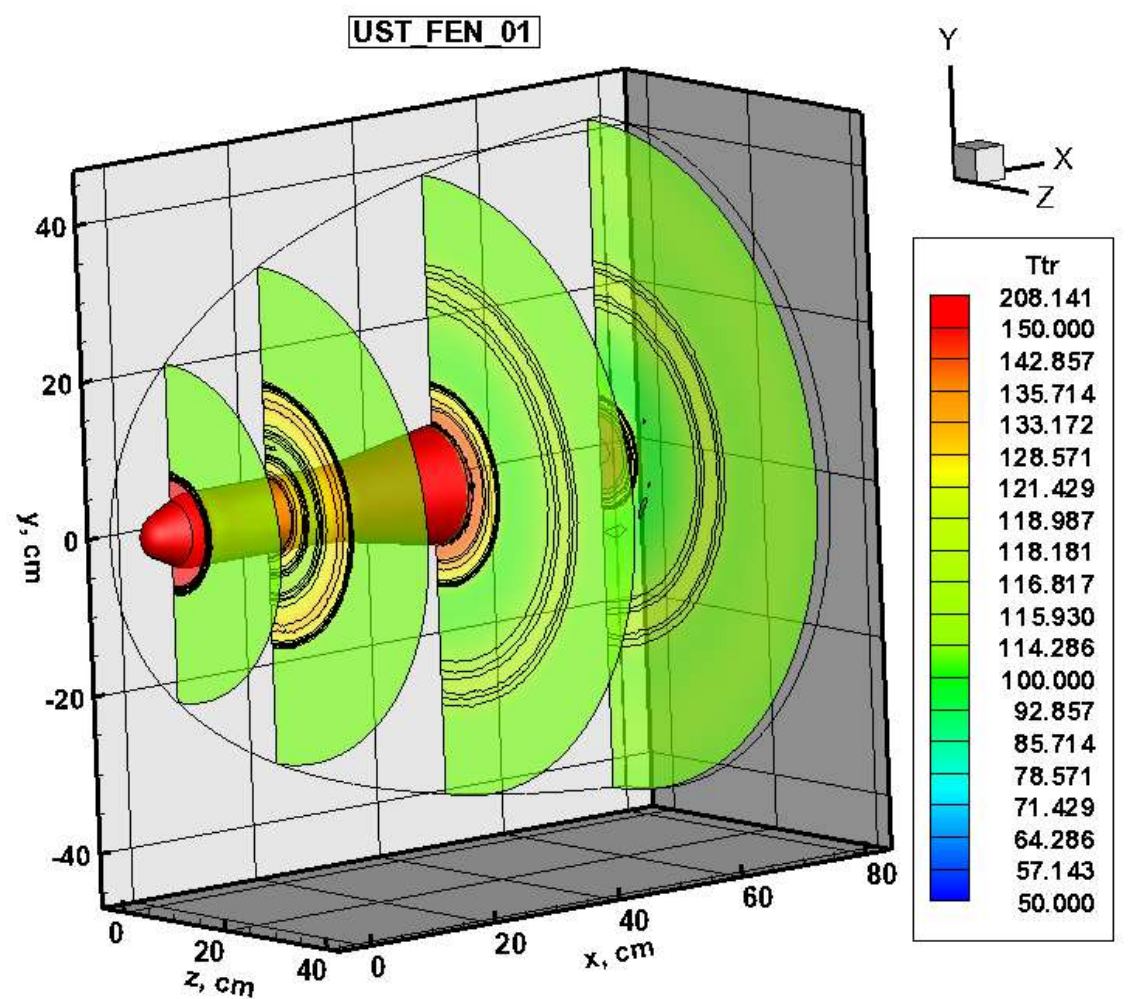

Рис. 6, б. Поле температуры в К при $\alpha=0$ в плоскостях перпендикулярных оси симметрии 
На рис. 6, в показано распределение давления. Здесь, как и на рис. $6, a$ обозначены 6 характерных зон: 1 - область в окрестности критической линии тока; 2 и 4 - ударные волны, генерируемые затупленной частью модели и юбкой; 3 - область разрежения над цилиндрической частью поверхности; 5 - область разрежения отрывного течения за юбкой и область осевого сжатия потока в следе (6).

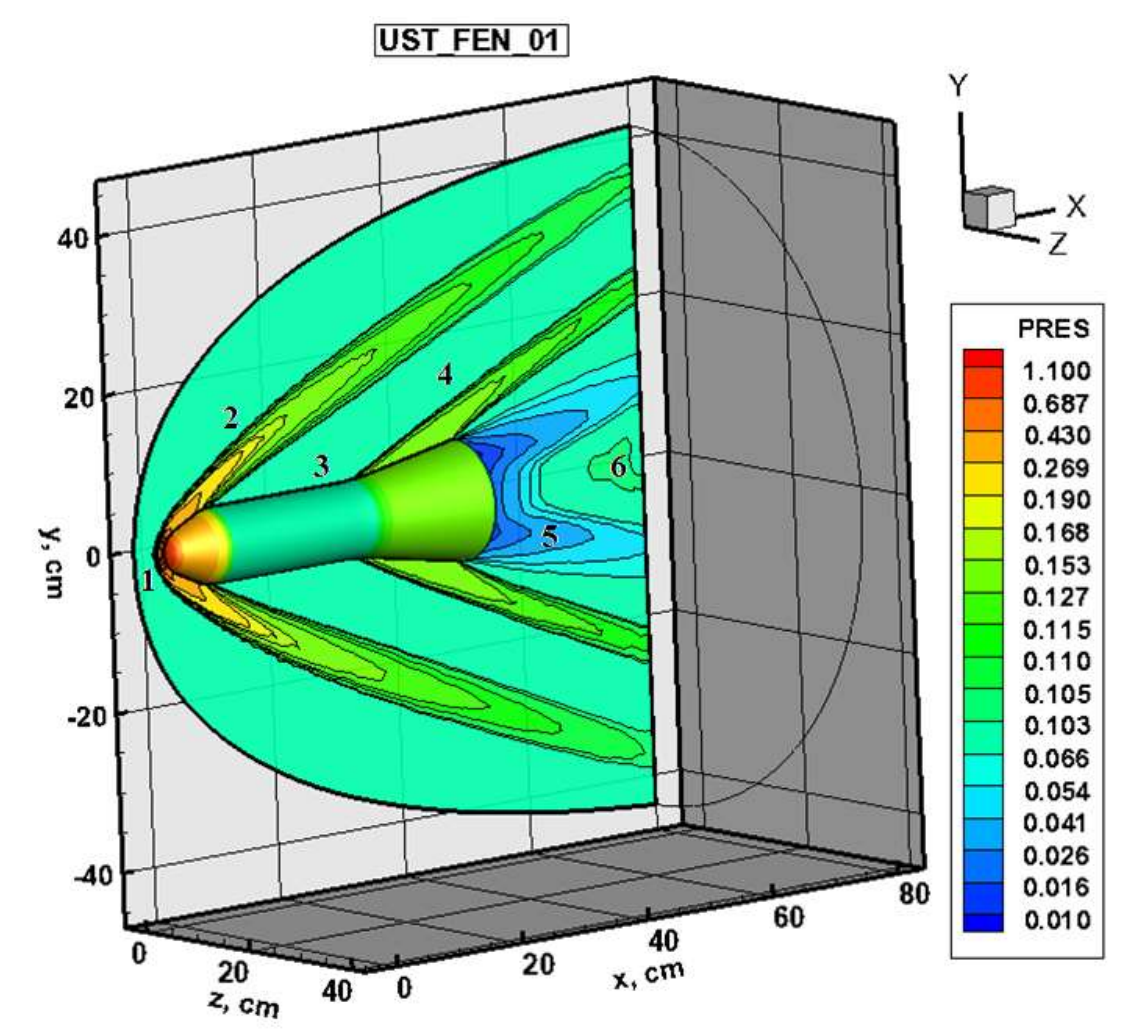

Рис. 6, в. Поле давления ( PRES $=\frac{p}{p_{0}}$ ) при $\alpha=0$ в плоскости симметрии $z=0$

Из рис. 6, $\partial$ видно, что в указанных выше характерных зонах происходит закономерное изменение чисел Маха: образование дозвуковых областей течения вблизи зоны полного торможения у лобовой поверхности области торможения и вблизи задней поверхности модели. Хорошо идентифицируются зоны торможения потока в двух ударных волнах с последующим разгоном газового потока, а также ускорение потока в области следа.

На рис. 6, ж показано распределение проекции скорости потока на продольную ось $x$, которое является хорошим дополнением к анализу распределений чисел Маха. Вблизи передней критической линии тока наблюдается торможение потока, а вблизи задней критической линии, в области возвратно-вихревого движения, скорость направлена навстречу основному газовому потоку. Здесь хорошо видна область возвратного движения, но при дополнительном анализе рис. 6,3 можно заключить, что получается на строго осесимметричное движение, а сильно неоднородный поток. Более того, в соответствии с [частное сообщение] при решении подобных задач в полной трехмерной постановке (без введения плоскости симметрии, как это сделано в данной работе), в расчете отрывного течения на неструктурированных сетках наблюдается полностью трехмерное и нестационарное решение.

Из рис. 6, б, г, е, з видно, что при осесимметричном течении не очень подробная неструктурированная расчетная сетка все же обеспечивает достаточно хорошее отображение симметричности численного решения. 
На рис. $6, u, \kappa$ показаны поля плотности и давления в плоскости симметрии $z=0$. Указанные данные позволяют в деталях проанализировать получающуюся симметрию численного решения (с небольшими отклонениями).

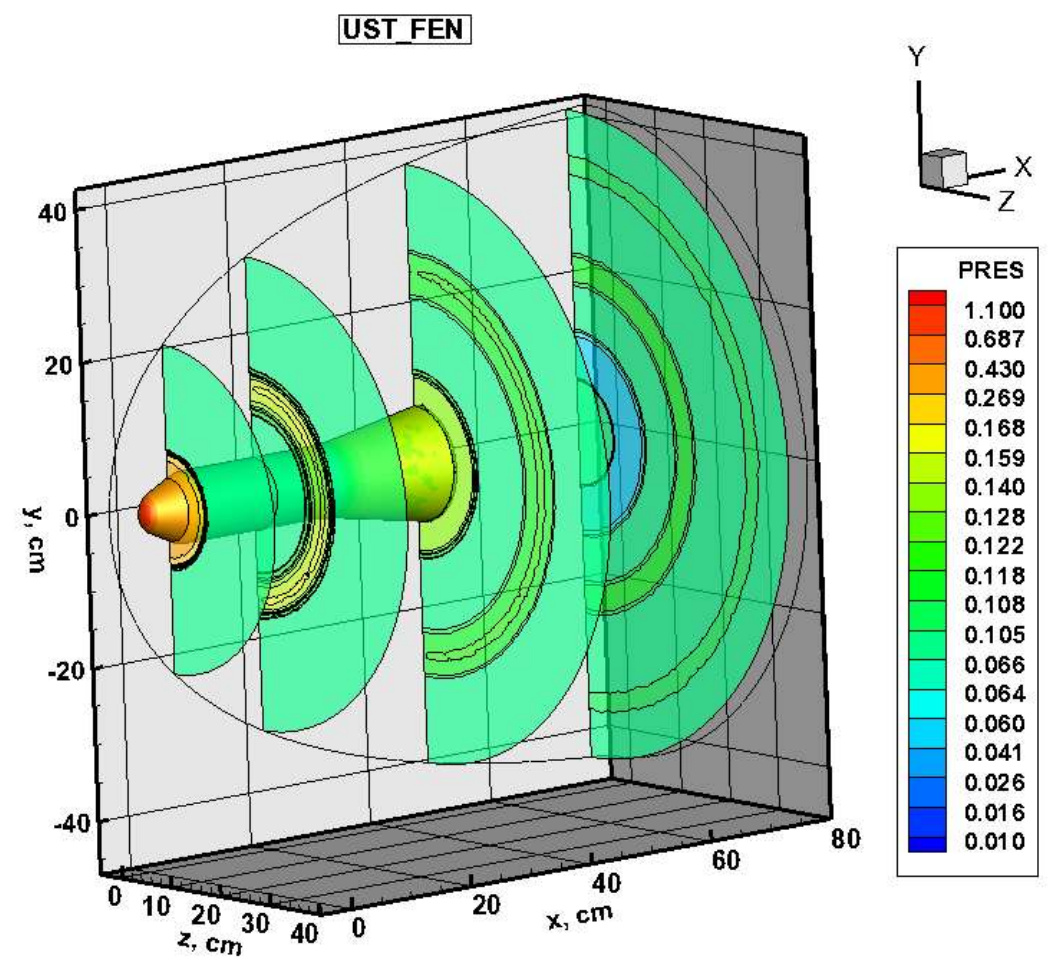

Рис. 6, г. Поле давления ( $\left.\mathrm{PRES}=p / p_{0}\right)$ при $\alpha=0$ в плоскостях перпендикулярных оси симметрии

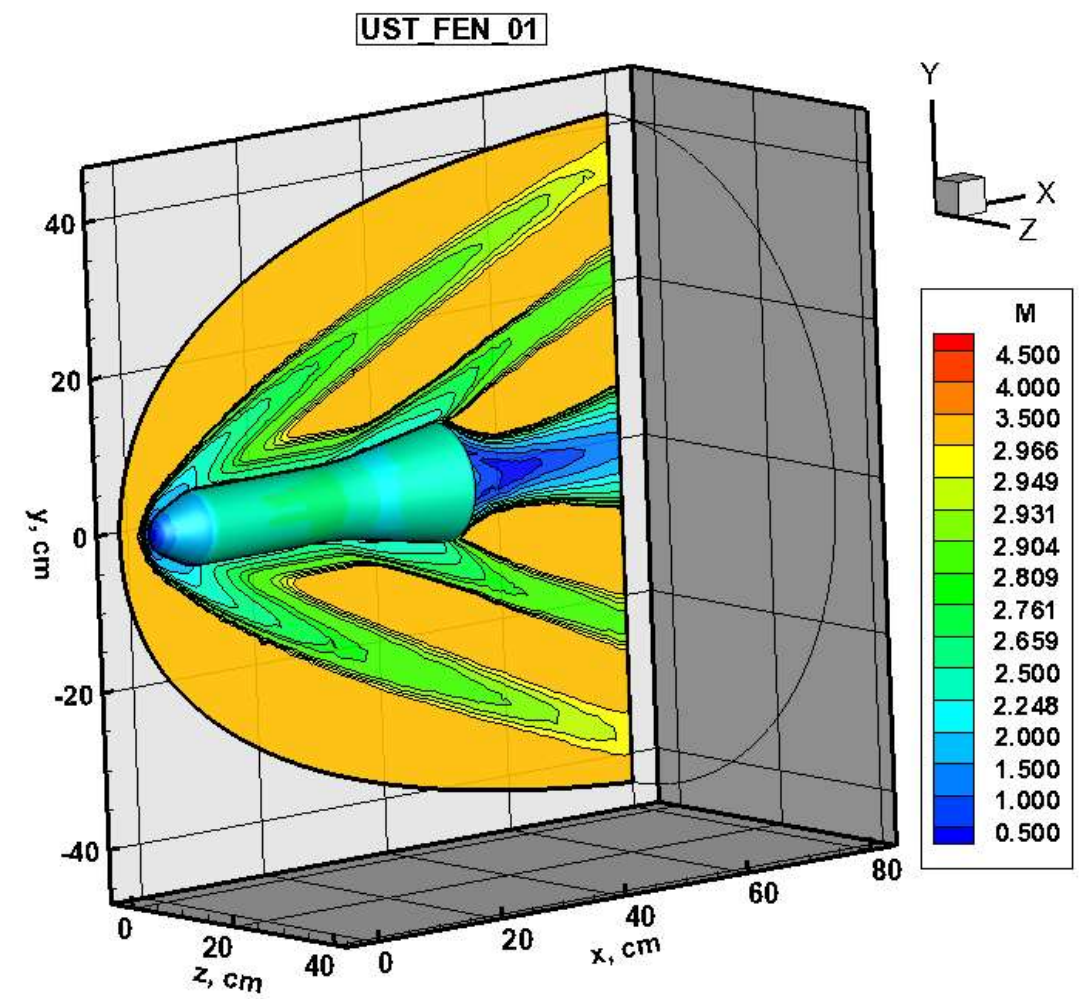

Рис. 6, $\partial$. Поле чисел Маха при $\alpha=0$ в плоскости симметрии $z=0$ 


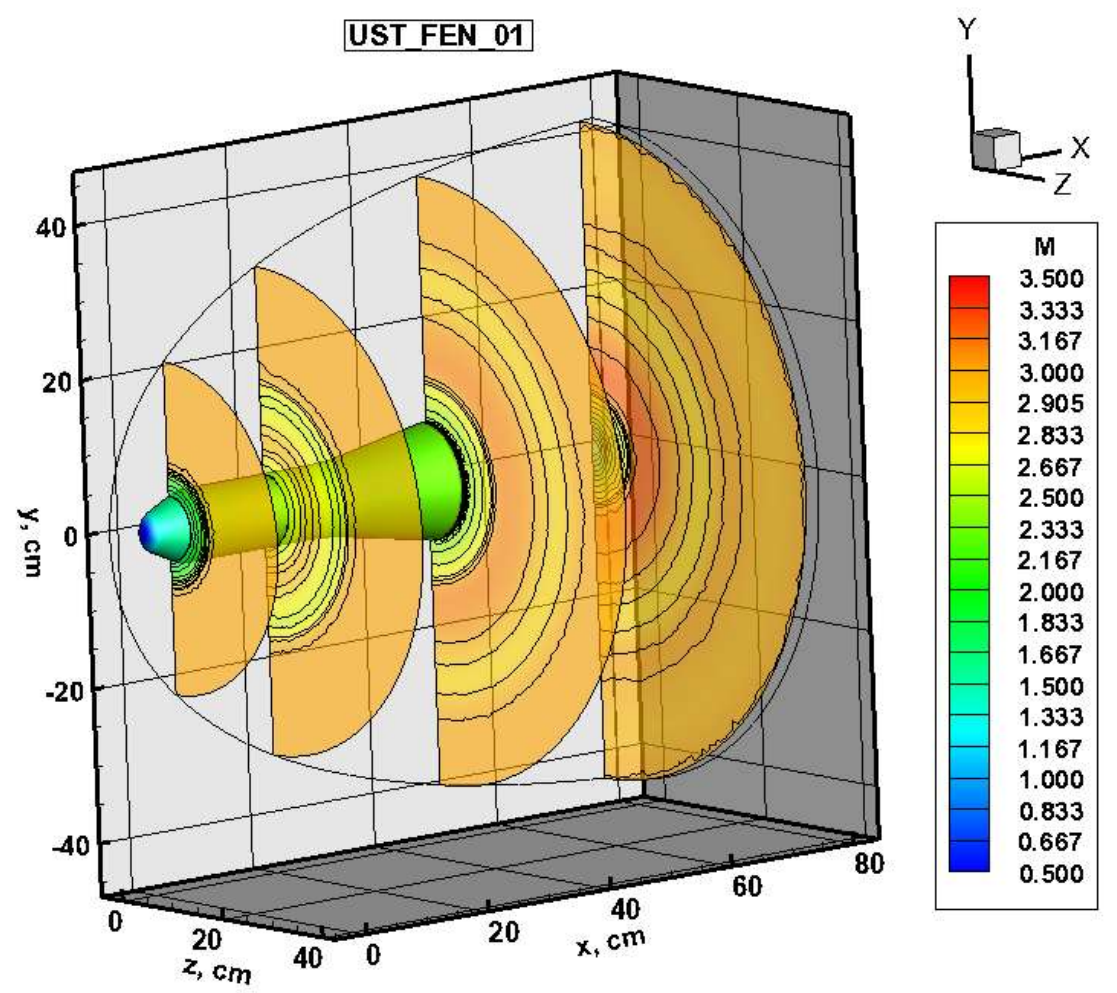

Рис. 6, e. Поле чисел Маха при $\alpha=0$ в плоскостях перпендикулярных оси симметрии

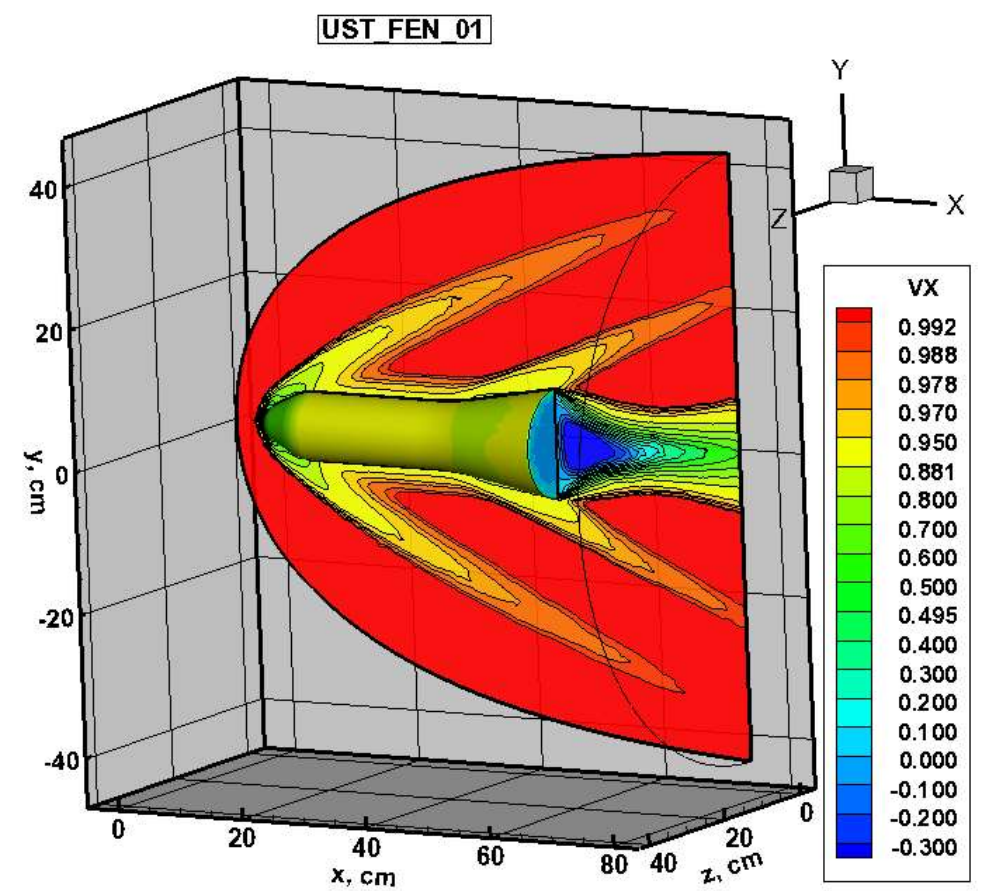

Рис. 6, ж. Поле продольной скорости $\frac{u}{u_{\infty}}$ при $\alpha=0$ в плоскости симметрии $z=0$ 


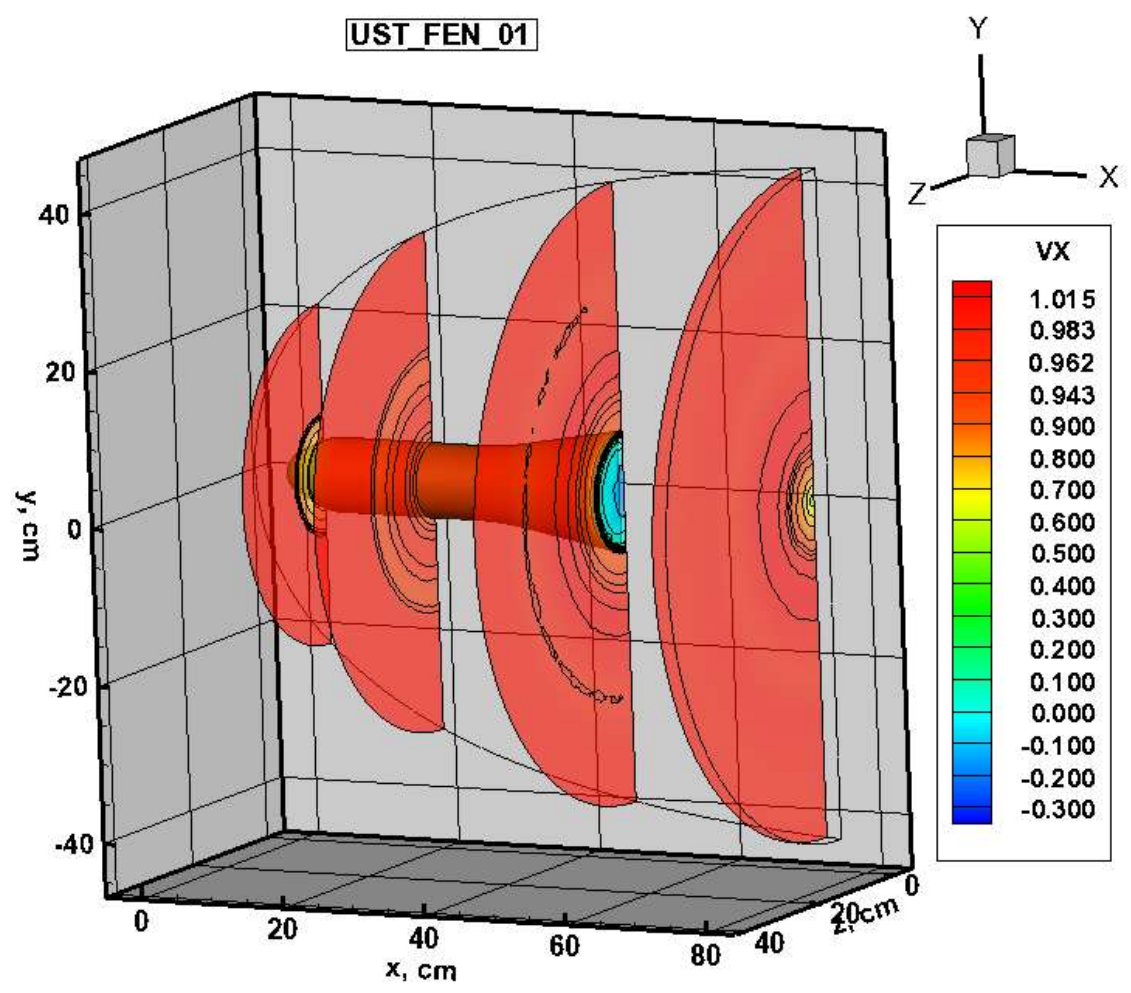

Рис. 6, з. Поле продольной скорости $\frac{u}{u_{\infty}}$ при $\alpha=0$ в плоскостях перпендикулярных оси симметрии
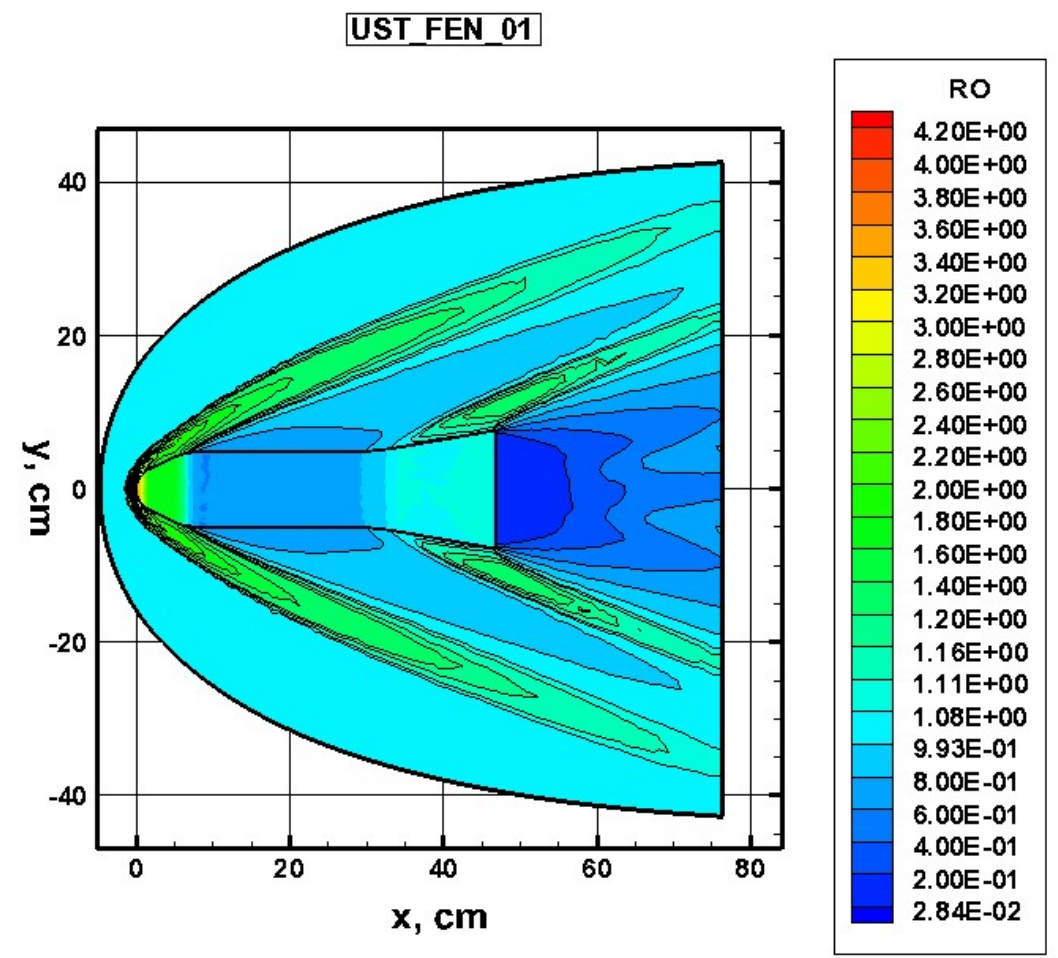

Рис. 6, $u$. Поле плотности $\frac{\rho}{\rho_{\infty}}$ при $\alpha=0$ в плоскости симметрии $z=0$ 


\section{UST_FEN_01}

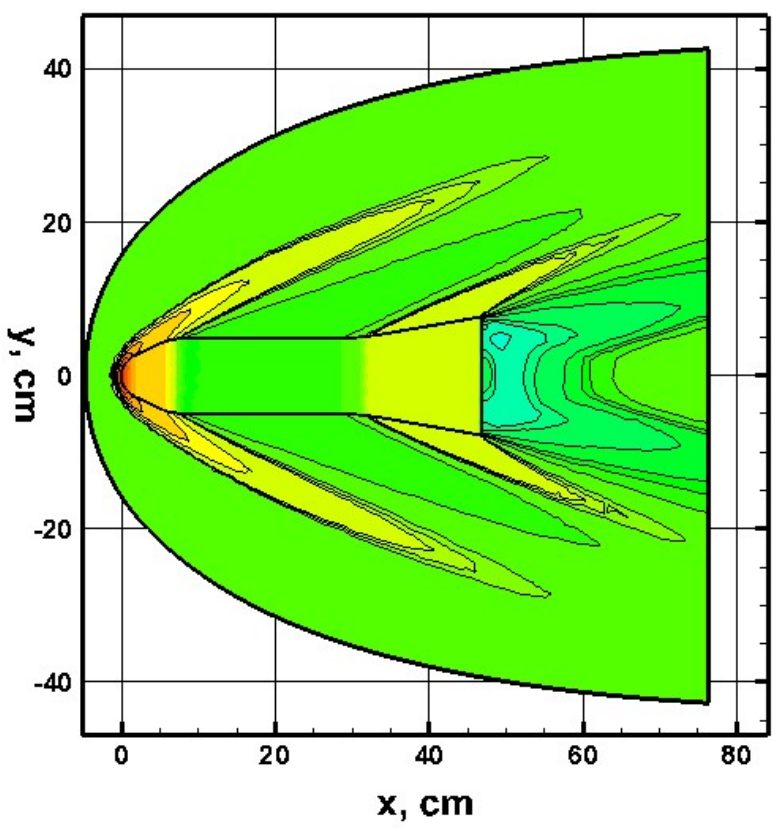

\section{PRES}

$1.15 \mathrm{E}+00$ 8.35E-01

6.06E-01

4.40E-01

3.19E-01

2.32E-01

1.68E-01

1.22E-01

1.18E-01

1.13E-01

8.87E-02

7.30E-02

6.44E-02

4.67E-02

2.46E-02

1.79E-02

$9.42 \mathrm{E}-03$

6.83E-03

4.96E-03

3. $60 \mathrm{E}-03$

2.61E-03

$1.90 \mathrm{E}-03$

1.38E-03

$1.00 \mathrm{E}-03$

Рис. 6, $к$. Поле давления ( PRES $=\frac{p}{p_{0}}$ ) при $\alpha=0$ в плоскости симметрии $z=0$

Заключительные два рисунка первой расчетной серии, рис. 7, $a$ и 7, 6 , демонстрируют распределение давления и температуры вдоль всей поверхности модели. Количественная информация данных рисунков позволяет проводит перекрестную верификацию компьютерных кодов.

На рис. 7, $a$ цифрами обозначены 7 характерных зон: 1 - зона торможения потока вблизи передней критической линии тока; 2 - область сжатия над конической поверхностью; 3 - область разрежения над цилиндрической поверхностью; 4 - область сжатия над конической юбкой; 6 - область разрежения над цилиндрической частью юбки; 7 - область разрежения у задней поверхности юбки.

UST FEN 01

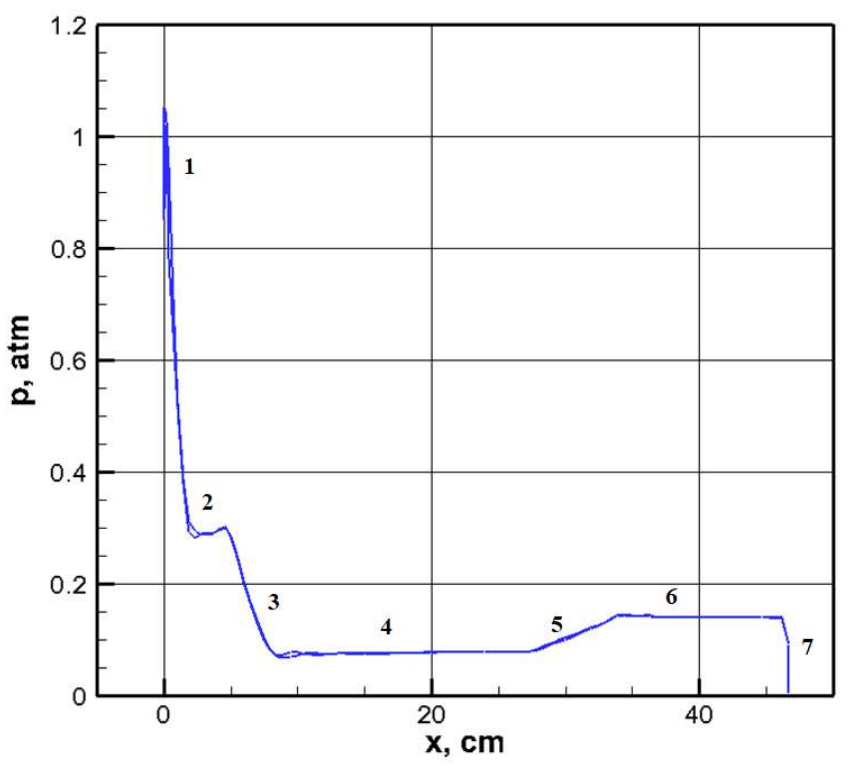

Рис. 7, a. Распределение давления вдоль поверхности при $\alpha=0$ в плоскости симметрии $z=0$ 


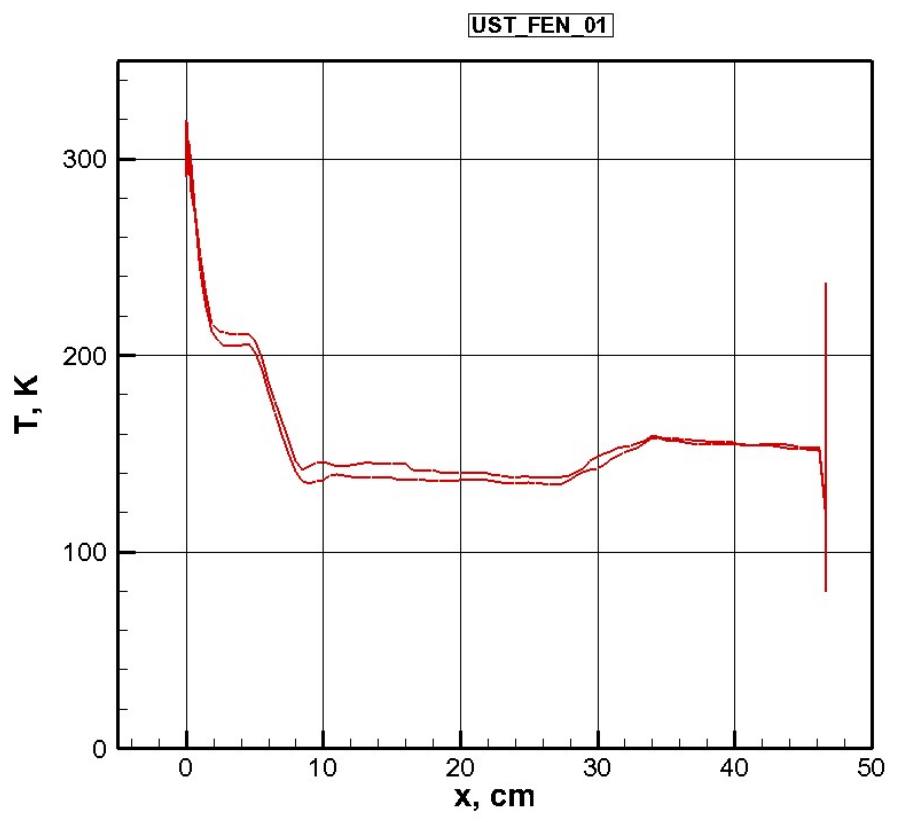

Рис. 7, б. Распределение температуры вдоль поверхности при $\alpha=0$ в плоскости симметрии $z=0$

Отметим три особенности показанных распределений. Во-первых, расчетные данные на верхней и нижней поверхности модели хорошо совпадают вследствие осесимметричного характера обтекания. Тем не менее, некоторые отличия (в первую очередь - в распределениях температуры) наблюдаются. Вторая особенность - резкое падение давление за срезом юбки. Физически это явление полностью обосновано, однако наименьшее достигаемое давление в разных настройках компьютерного кода дают разные величины. В-третьих, сильная осцилляция температуры за срезом юбки. Здесь находит отражение тот факт, что сразу за срезом юбки температура резко падает, а вблизи задней критической линии тока - возрастает, поскольку встречный поток газа тормозится о поверхность.

На двух последующих сериях рисунков показаны расчетные данные для углов атаки $\alpha=4^{\circ}$ и $8^{\circ}$. Четко прослеживается отклонение поля течения от аксиальной симметрии. На pис. $8, \sigma, 2, e$, з и 10, $\sigma, 2, e$, з наблюдается серпообразная структура течения во всех поперечных сечениях. При увеличении угла атаки указанная степень отклонения становится все более очевидной. При угле атаки $\alpha=8^{\circ}$ на подветренной стороне цилиндрической поверхности хорошо видна область разряжения (рис. 10,2 ), отвечающая сходу потока с поверхности. Об этом свидетельствует также распределение температуры (рис. 10, б) в поперечных сечениях потока. На распределениях газодинамических функций за срезом юбки видна серпообразная структура течения, обусловленная несимметричностью обтекания.

На рис. 9 и 11 показаны распределения давления и температуры вдоль поверхности в плоскости симметрии $z=0$, где кривые на нижней и верхней частях поверхности закономерно расходятся.

Подводя итог анализу расчетных данных, полученных с использованием неструктурированной сетки, отметим хорошее совпадение с экспериментальными данными и отчетливое разрешение всех характерных особенностей течения.

На рис. 12-16 представлены аналогичные численные данные, но полученные с использованием компьютерного кода PERAT-3D. Первое, что несомненно можно отметить - это более четкое описание всех фронтов получаемых численных решениях. Все отмеченные выше характерные области течения здесь также хорошо видны. На рисунках с распределениями газодинамических функций в поперечных сечениях видны гладкие серпообразные структуры при течении под углом атаки. Хорошее разрешение течения в области отрыва позволяет идентифицировать внутреннюю структуру возвратно-вихревого течения, например, ускорение встречного потока (рис. 12, $\kappa, 14, \kappa$ и 16, $\kappa$ ). 
По причине использования многоблочной структуры расчетной сетки удается достаточно просто выполнить оценку относительной величины в распределениях давления и теплового потока вдоль обтекаемой модели. На рис. 13,15 и 17 даны распределения давления вдоль поверхности испытуемой модели в плоскости симметрии $z=0$.Для удобства идентификации распределение давления на разных участках помечены разными цветами.

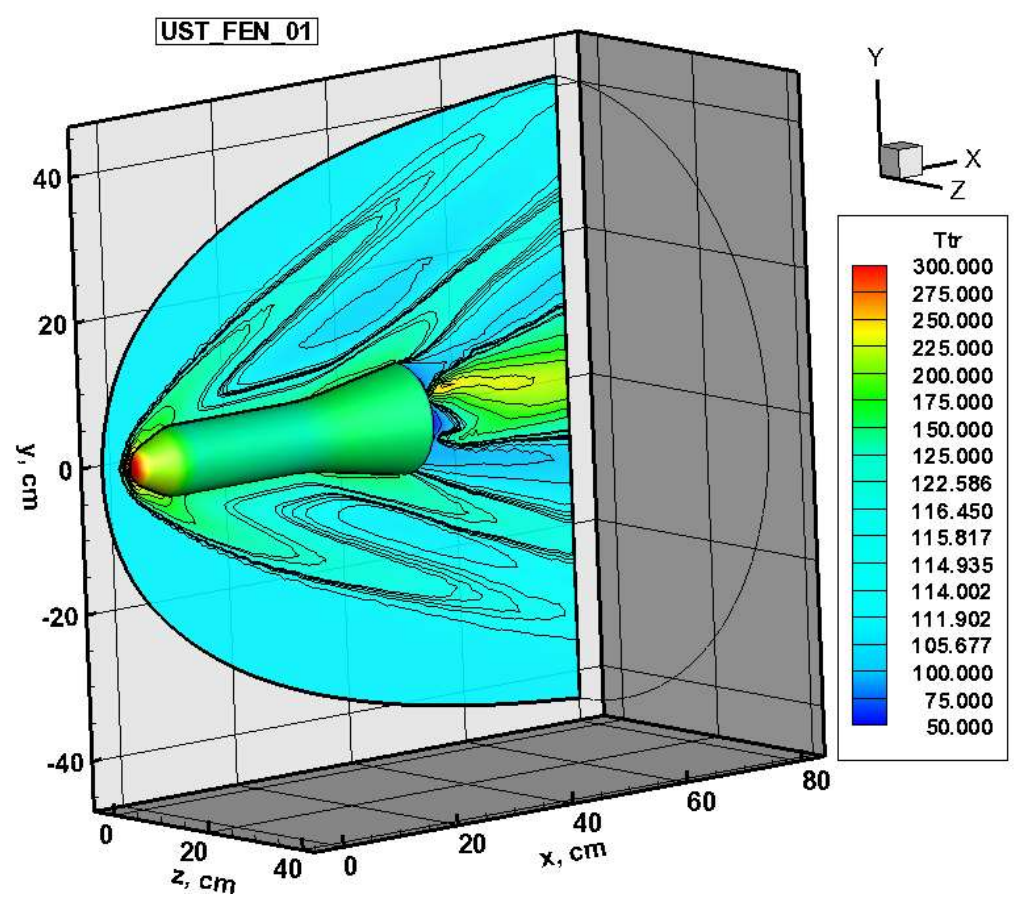

Рис. 8, $a$. Поле температуры в К при $\alpha=4^{\circ}$ в плоскости симметрии $z=0$

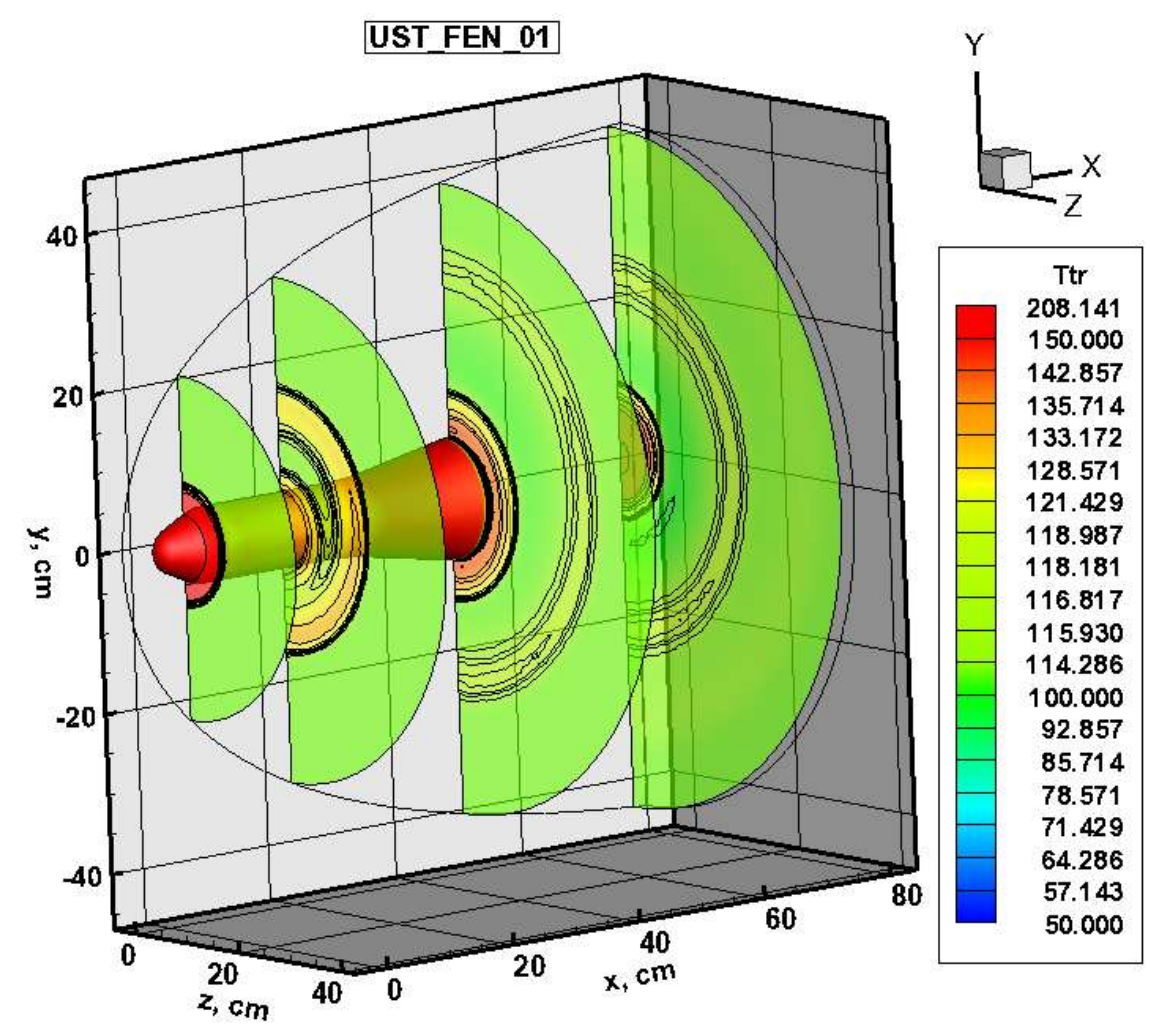

Рис. 8, б. Поле температуры в К при $\alpha=4^{\circ}$ в плоскостях перпендикулярных оси симметрии 


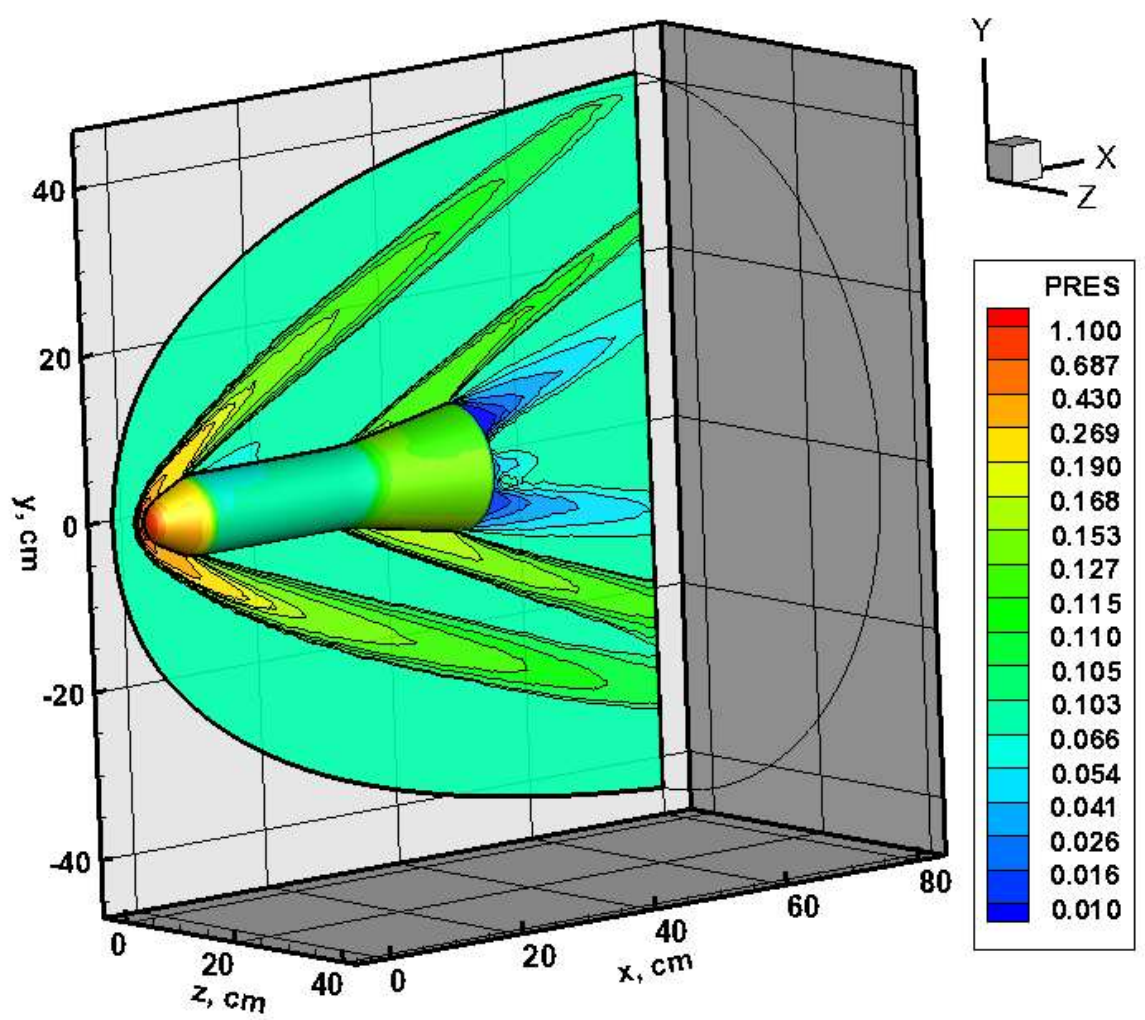

Рис. 8, в. Поле давления ( PRES $=\frac{p}{p_{0}}$ ) при $\alpha=4^{\mathrm{o}}$ в плоскости симметрии $z=0$

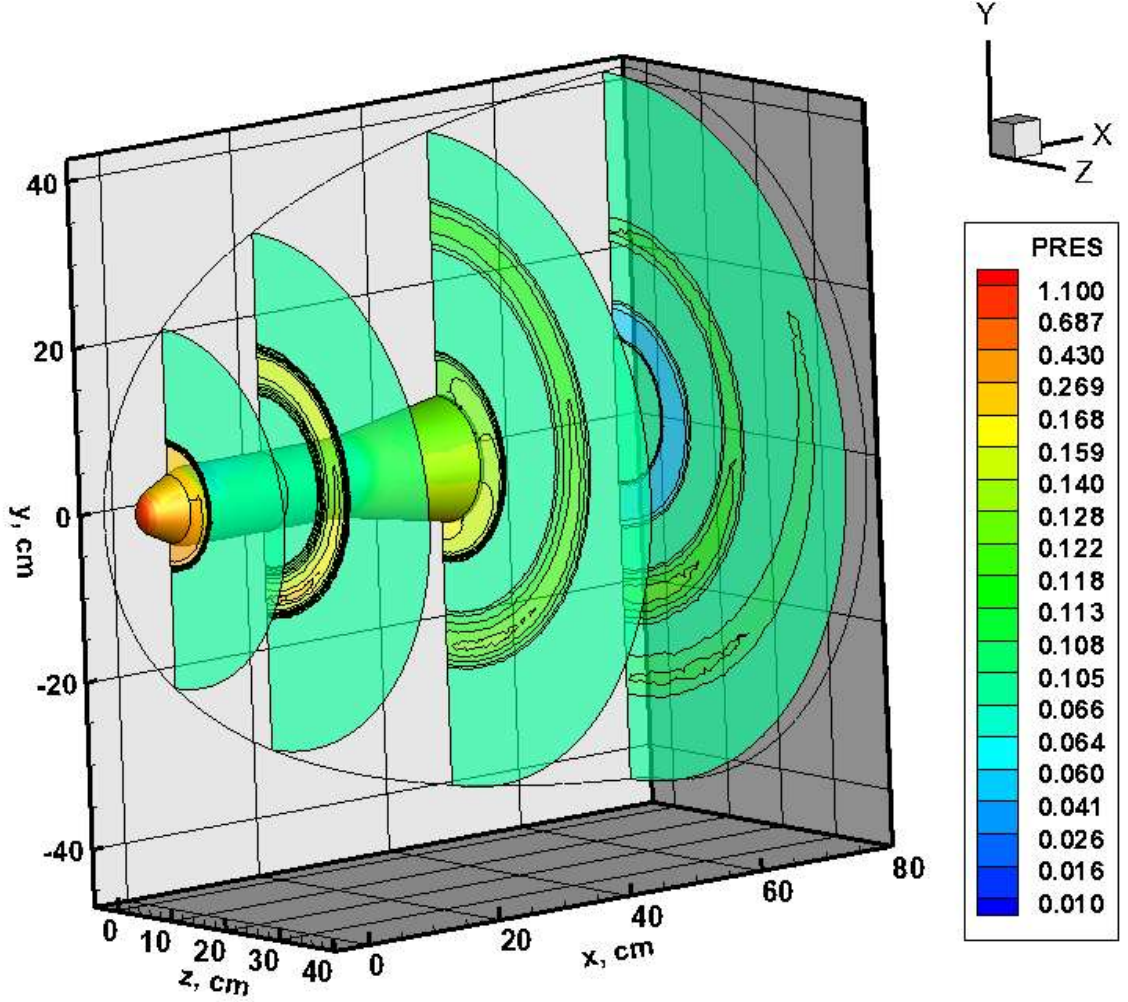

Рис. 8 , г. Поле давления $\left(\mathrm{PRES}=\frac{p}{p_{0}}\right)$ при $\alpha=4^{\mathrm{o}}$ в плоскостях перпендикулярных оси симметрии 


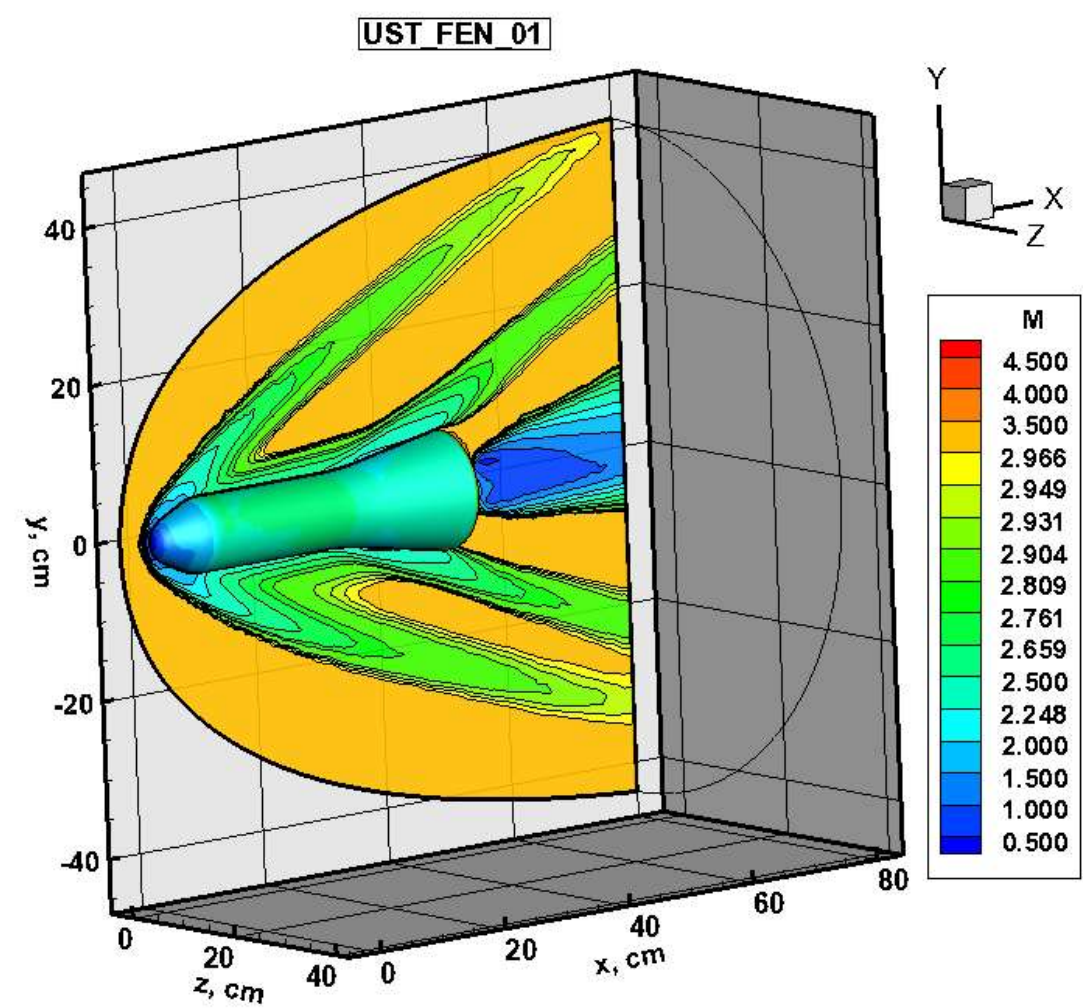

Рис. $8, \partial$. Поле чисел Маха при $\alpha=4^{\circ}$ в плоскости симметрии $z=0$
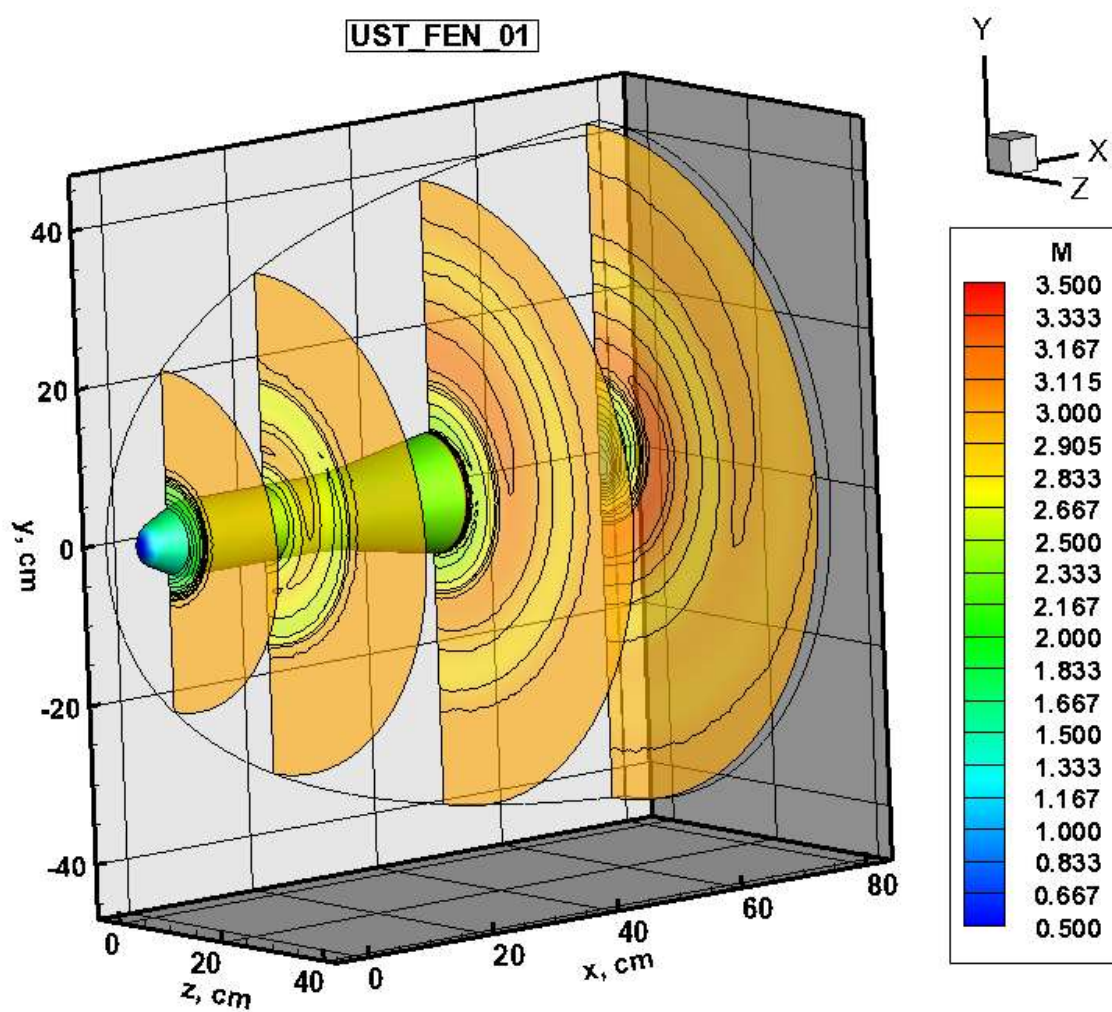

Рис. 8, e. Поле чисел Маха при $\alpha=4^{\circ}$ в плоскостях перпендикулярных оси симметрии 


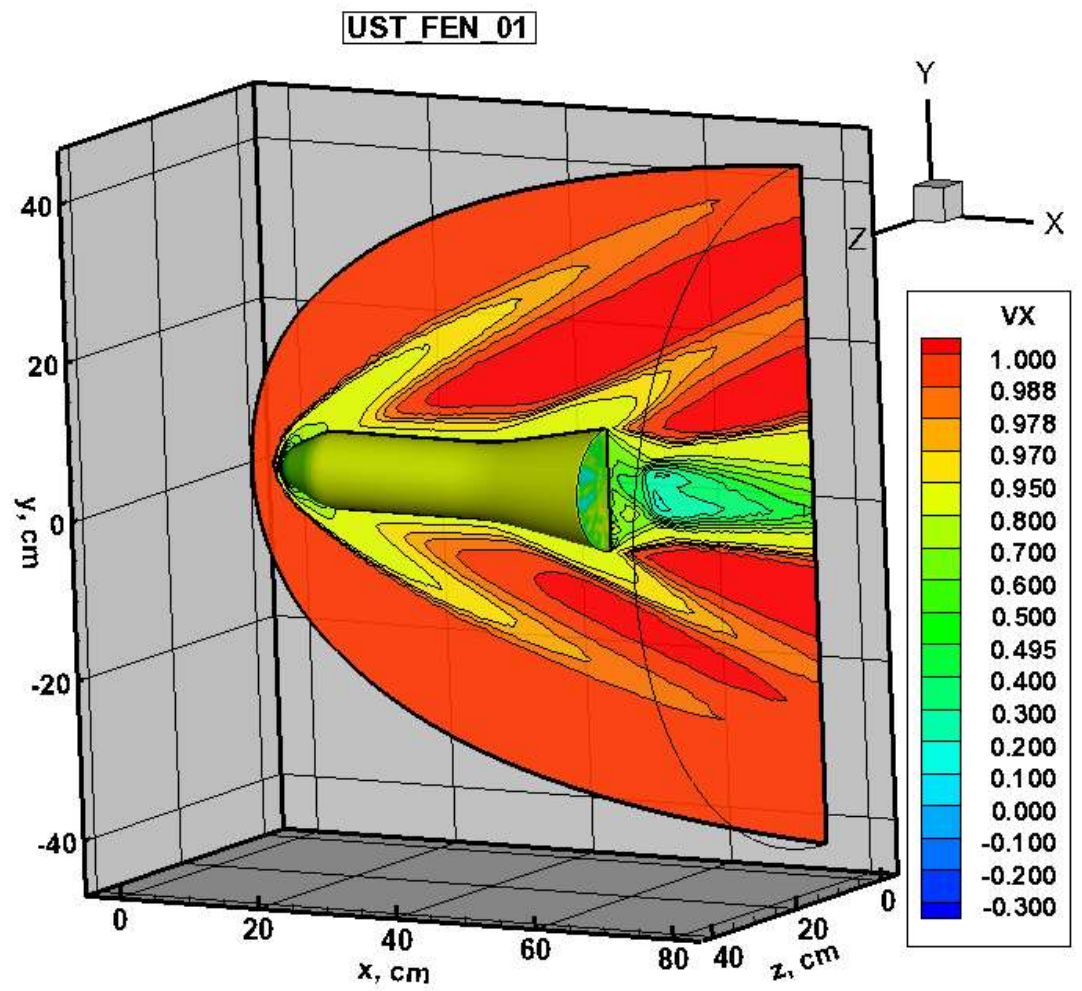

Рис. 8 , ж. Поле продольной скорости $\frac{u}{u_{\infty}}$ при $\alpha=4^{\circ}$ в плоскости симметрии $z=0$

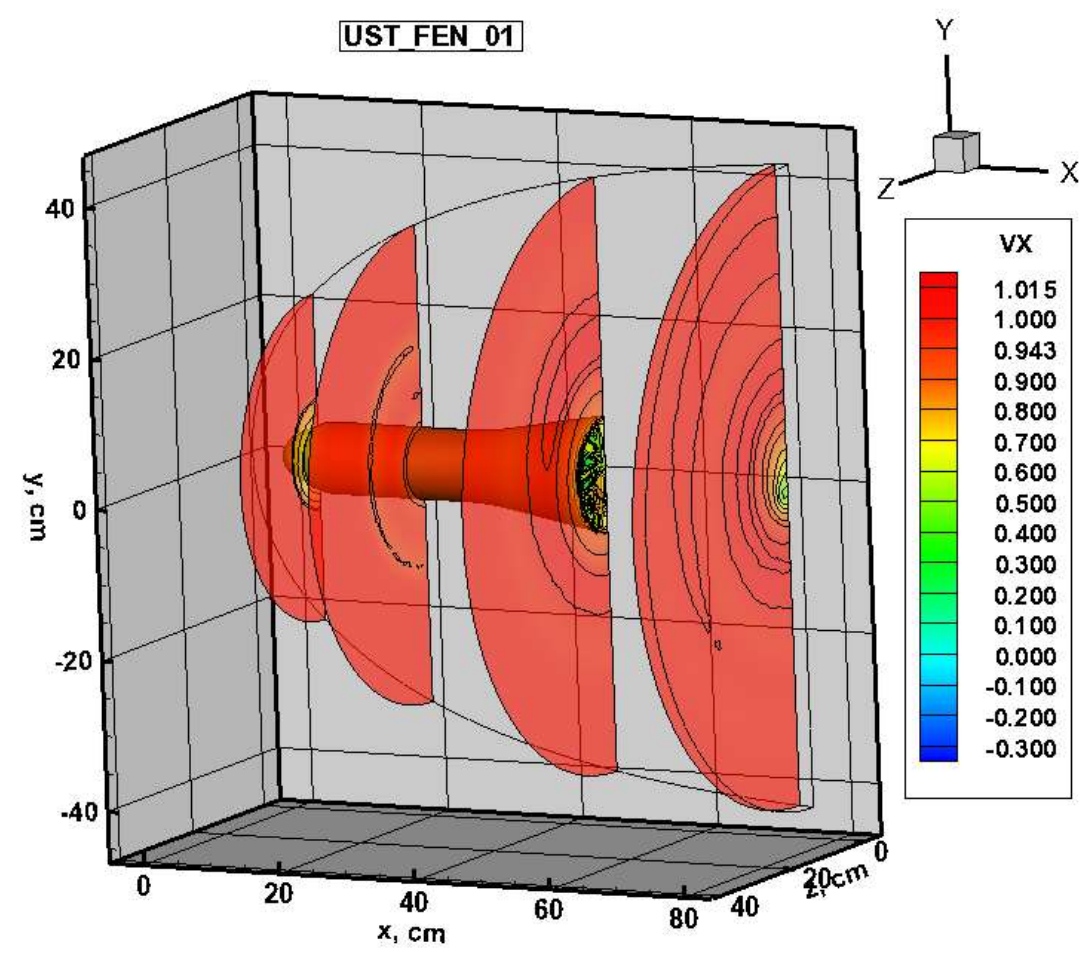

Рис. 8, з. Поле продольной скорости $\frac{u}{u_{\infty}}$ при $\alpha=4^{\circ}$ в плоскостях перпендикулярных оси симметрии 


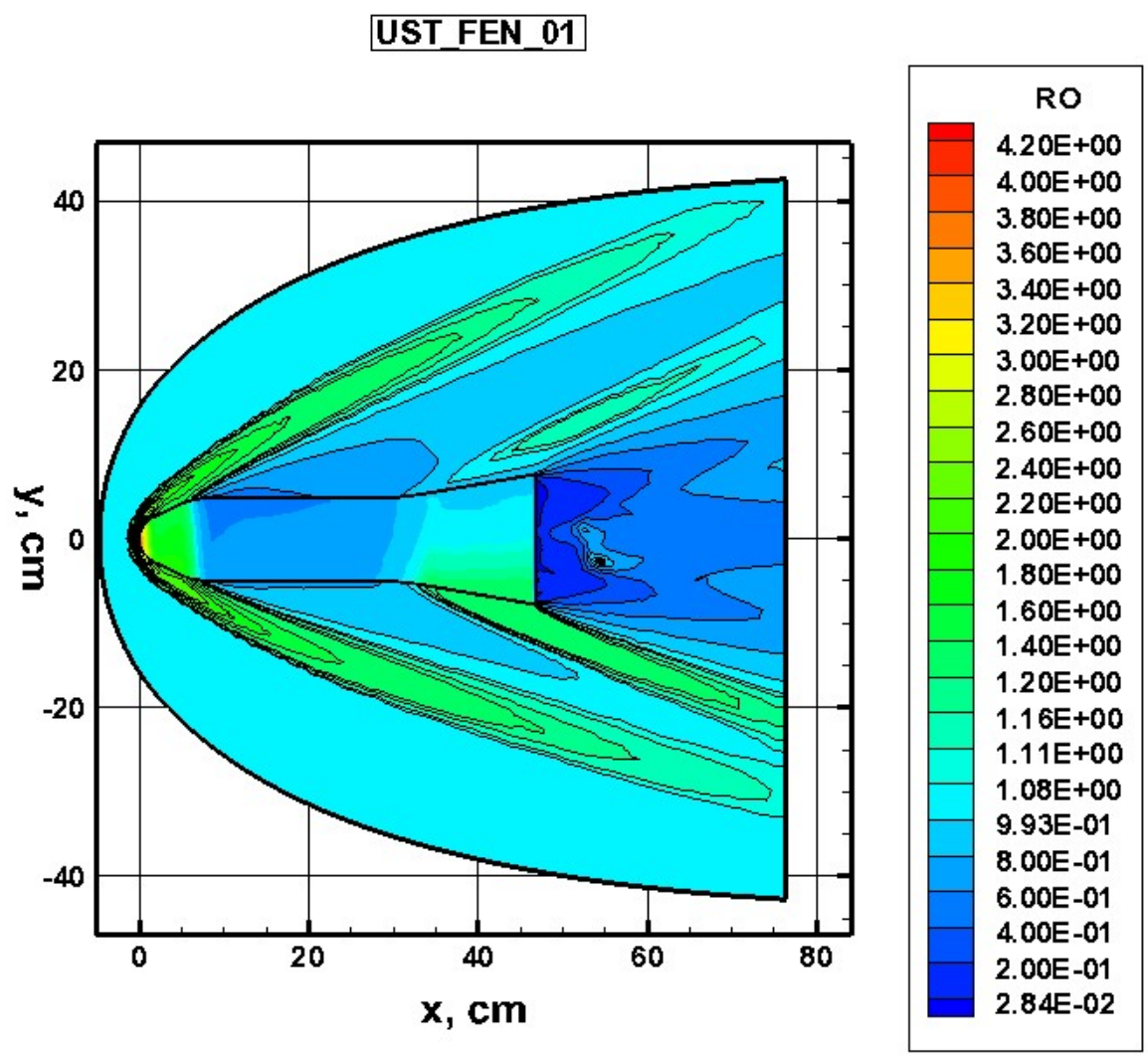

Рис. $8, u$. Поле плотности $\frac{\rho}{\rho_{\infty}}$ при $\alpha=4^{\circ}$ в плоскости симметрии $z=0$

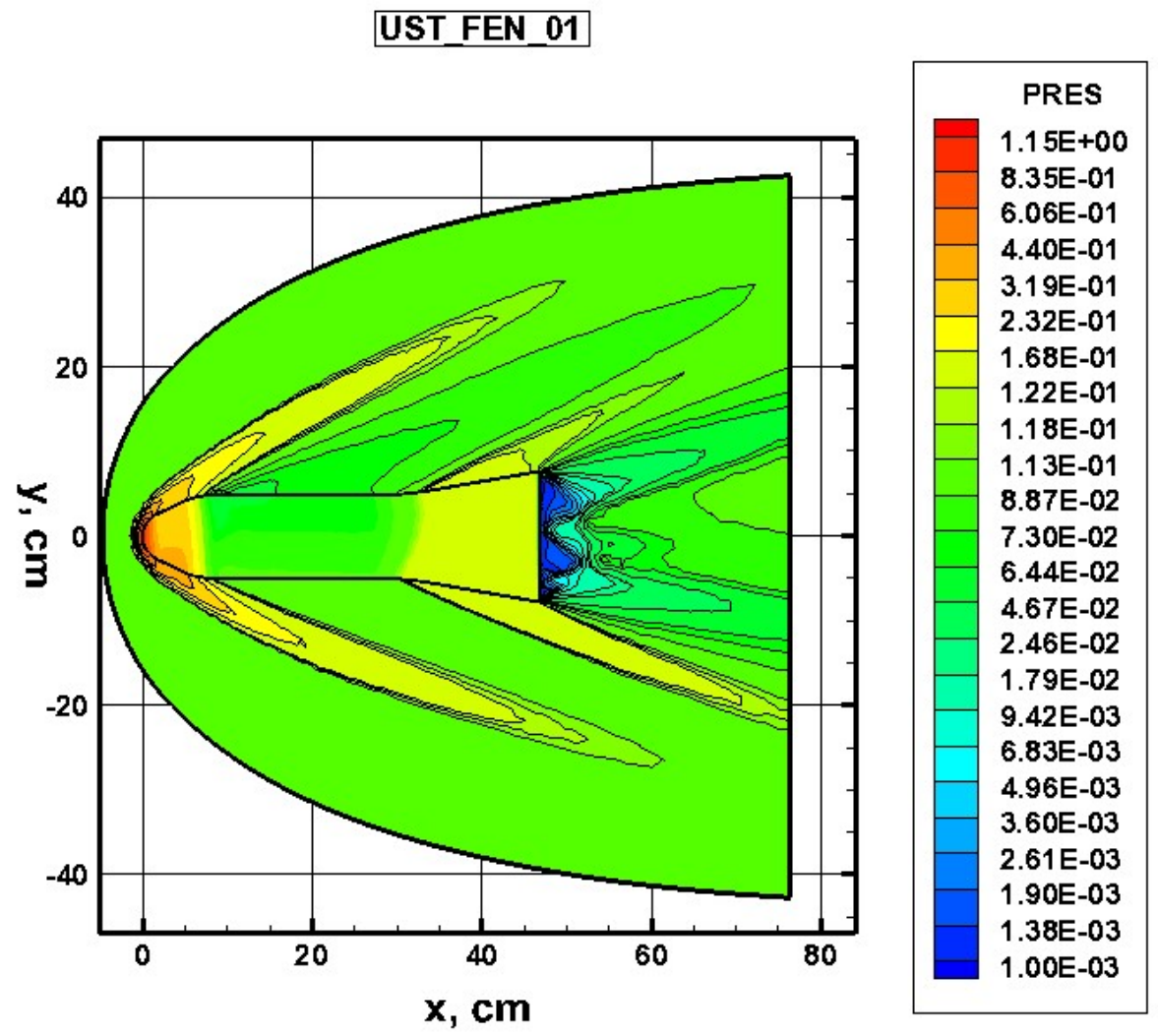

Рис. $8, \kappa$. Поле давления ( PRES $=\frac{p}{p_{0}}$ ) при $\alpha=4^{\circ}$ в плоскости симметрии $z=0$ 


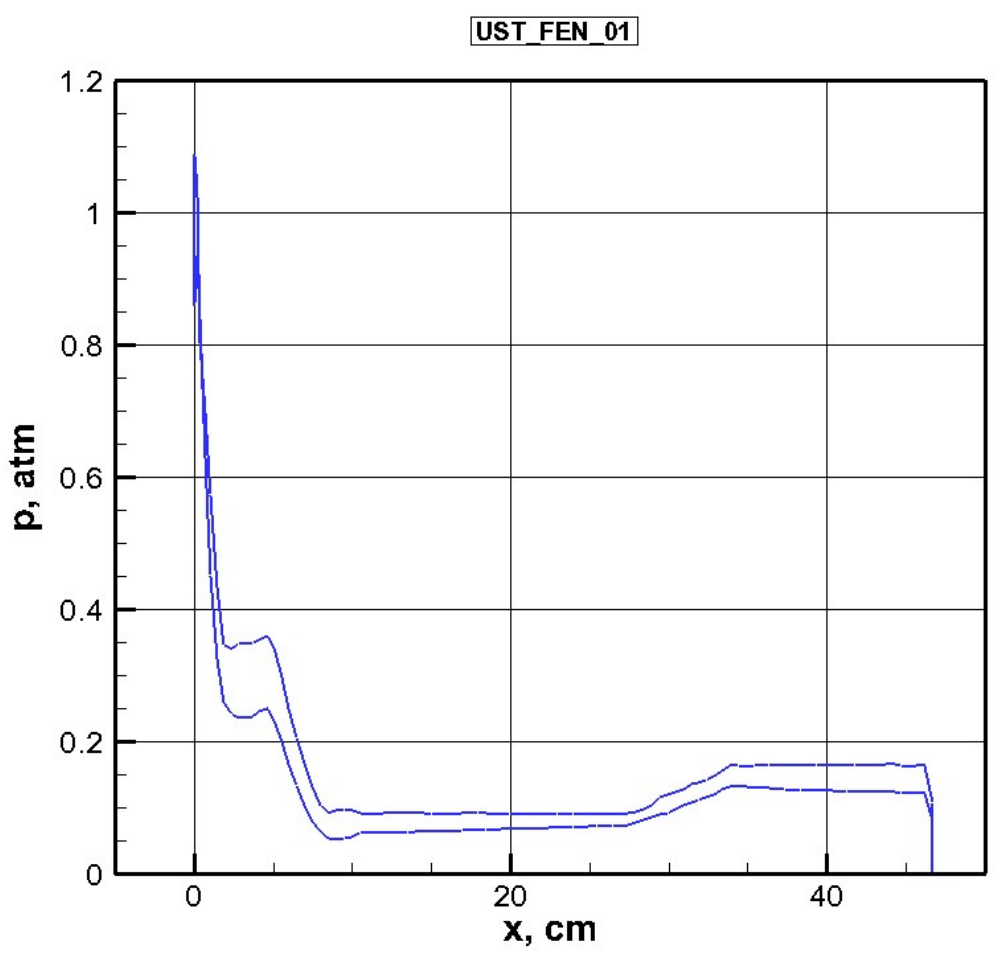

Рис. 9, a. Распределение давления вдоль поверхности при $\alpha=4^{\circ}$ в плоскости симметрии $z=0$

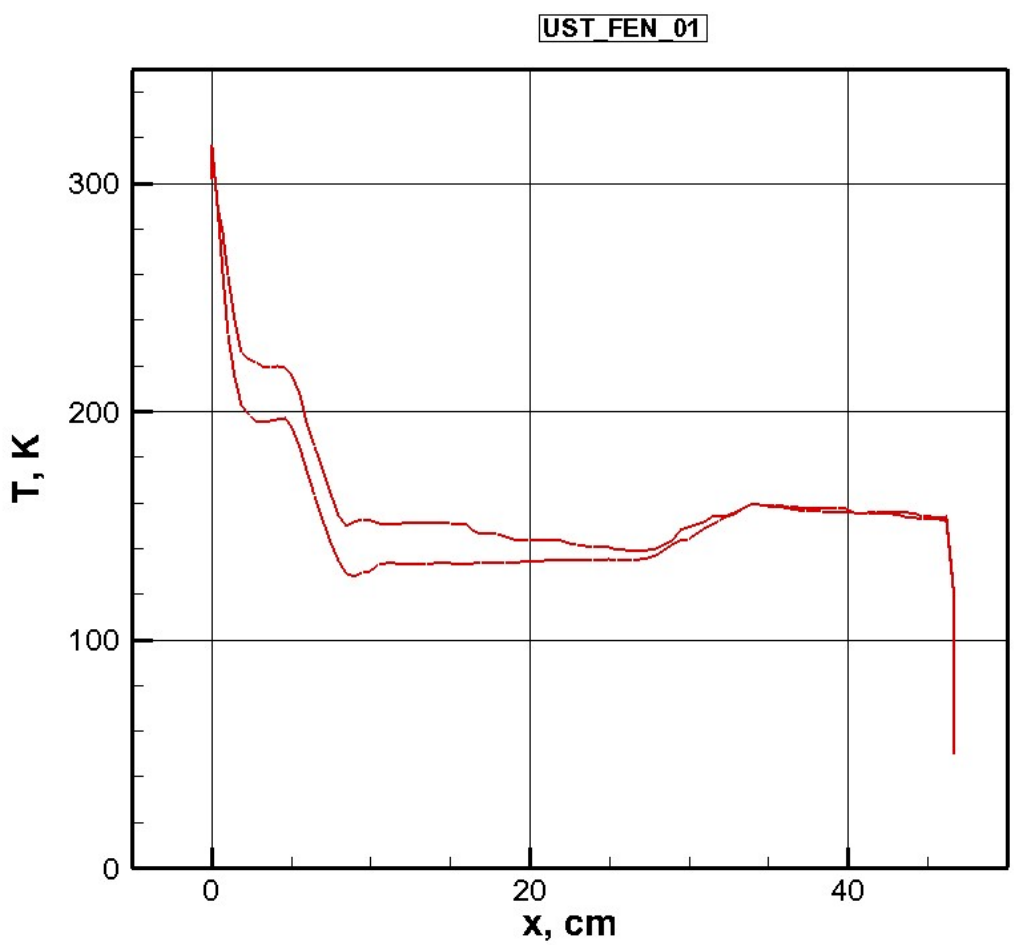

Рис. 9, б. Распределение температуры вдоль поверхности при $\alpha=4^{\circ}$ в плоскости симметрии $z=0$ 


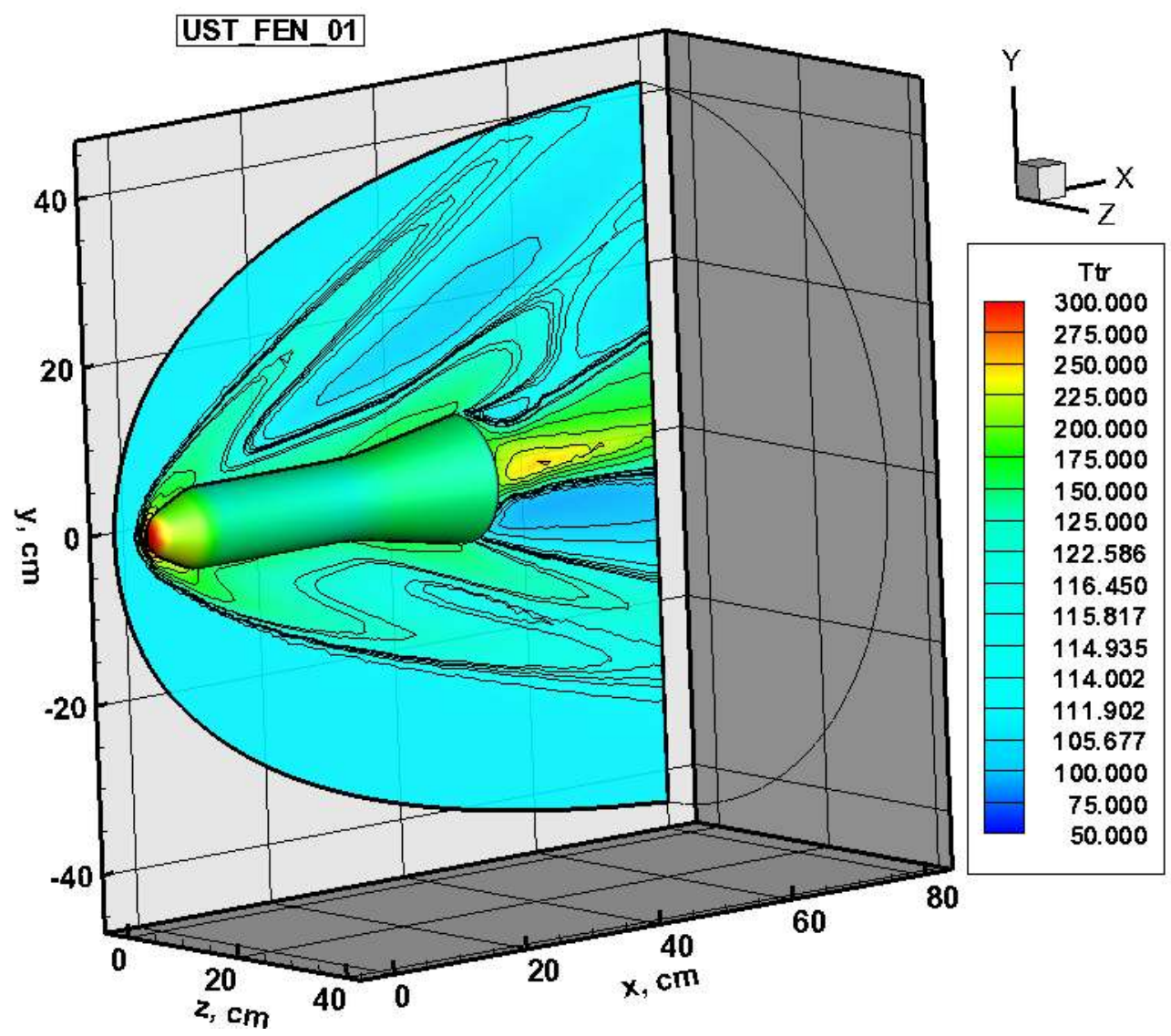

Рис. 10, $a$. Поле температуры в К при $\alpha=8^{\circ}$ в плоскости симметрии $z=0$

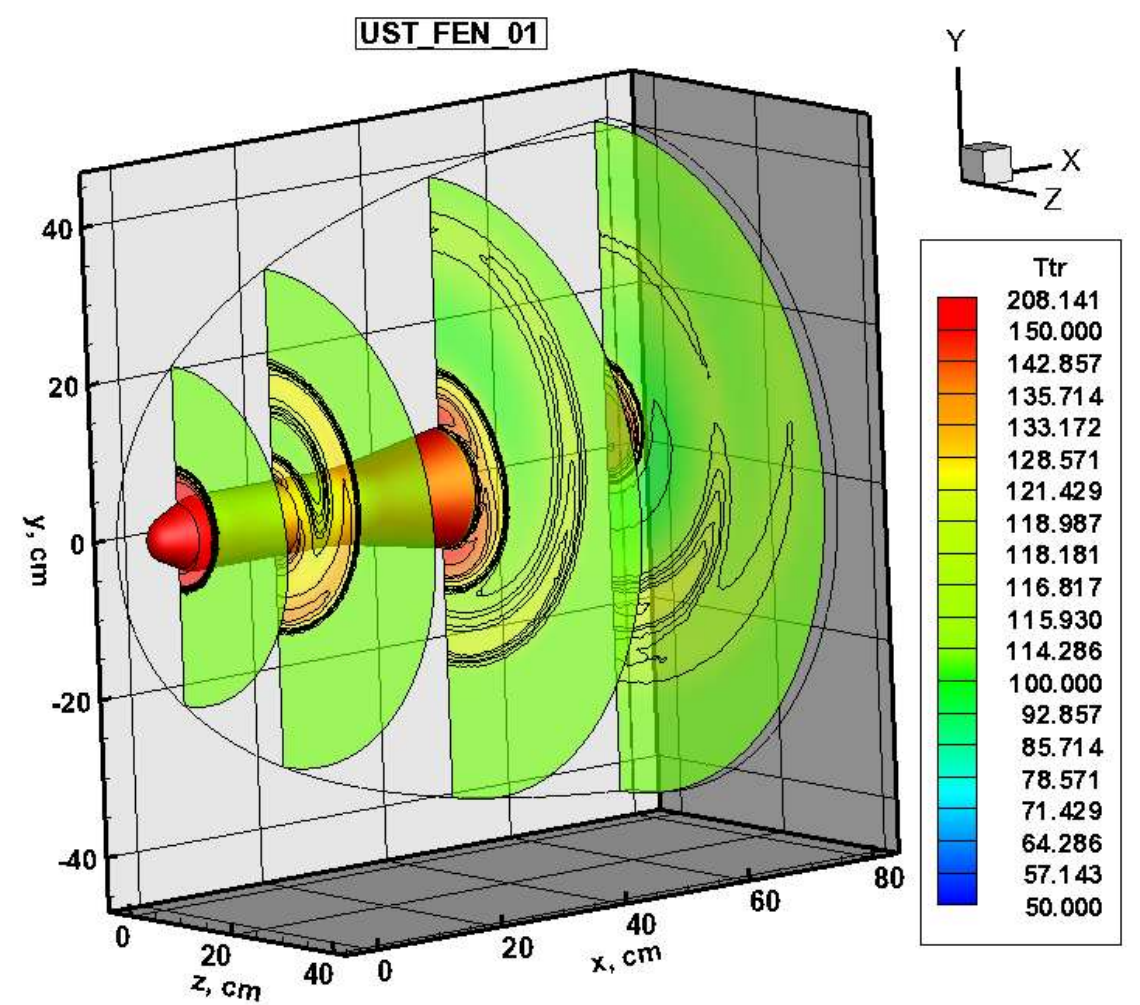

Рис. 10, б. Поле температуры в К при $\alpha=8^{\circ}$ в плоскостях перпендикулярных оси симметрии 


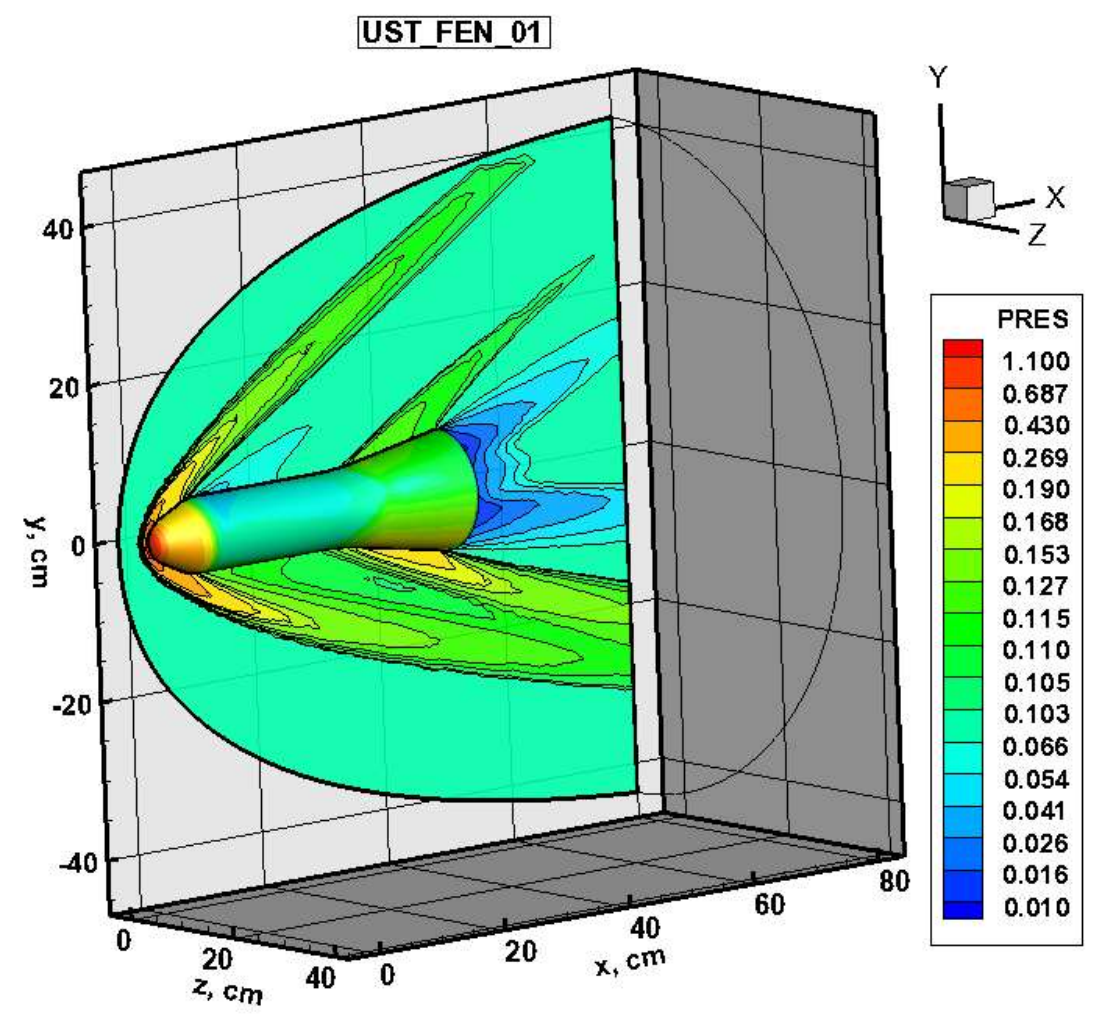

Рис. 10, в. Поле давления $\left(\right.$ PRES $\left.=\frac{p}{p_{0}}\right)$ при $\alpha=8^{\circ}$ в плоскости симметрии $z=0$

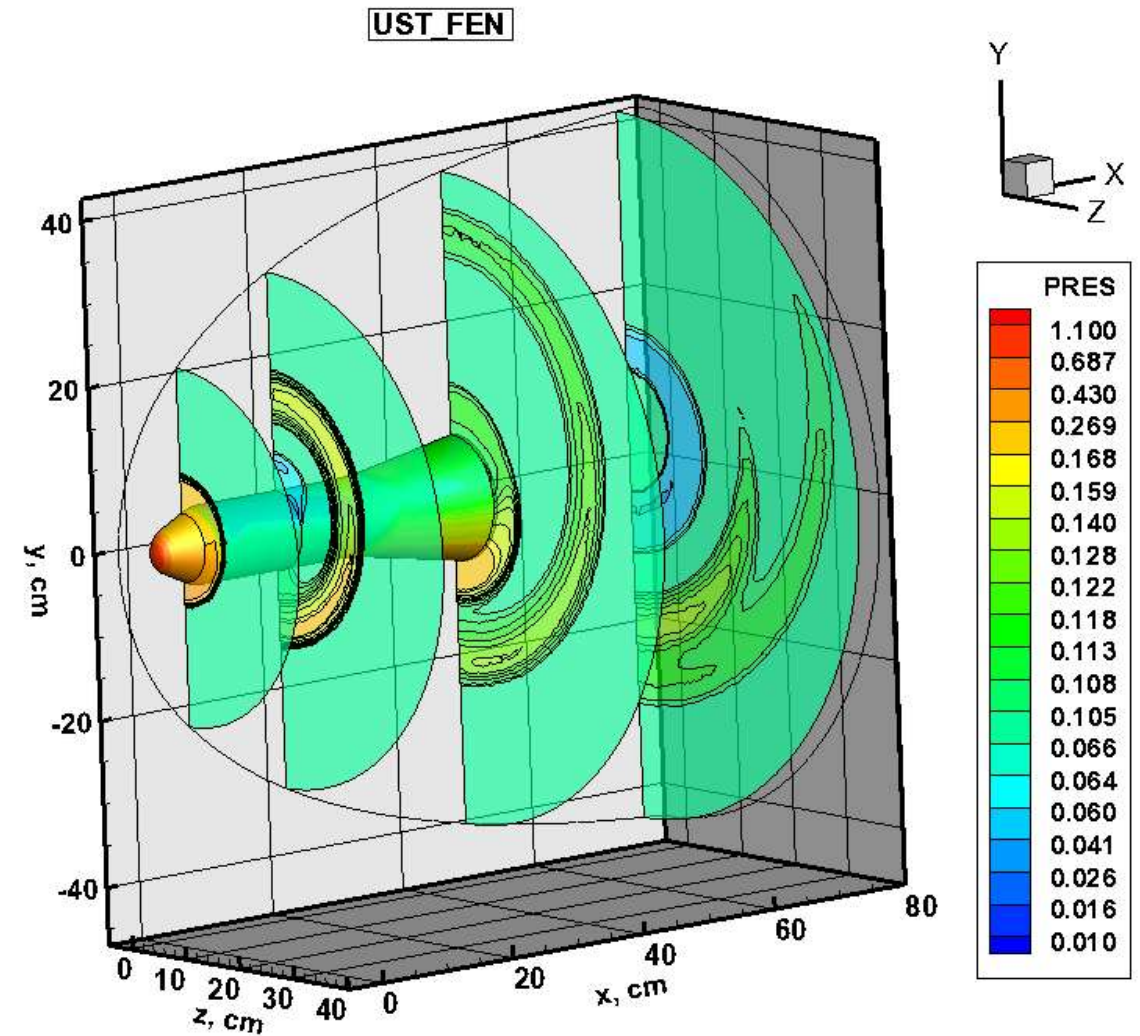

Рис. 10, г. Поле давления ( $\mathrm{PRES}=\frac{p}{p_{0}}$ ) при $\alpha=8^{\circ}$ в плоскостях перпендикулярных оси симметрии 


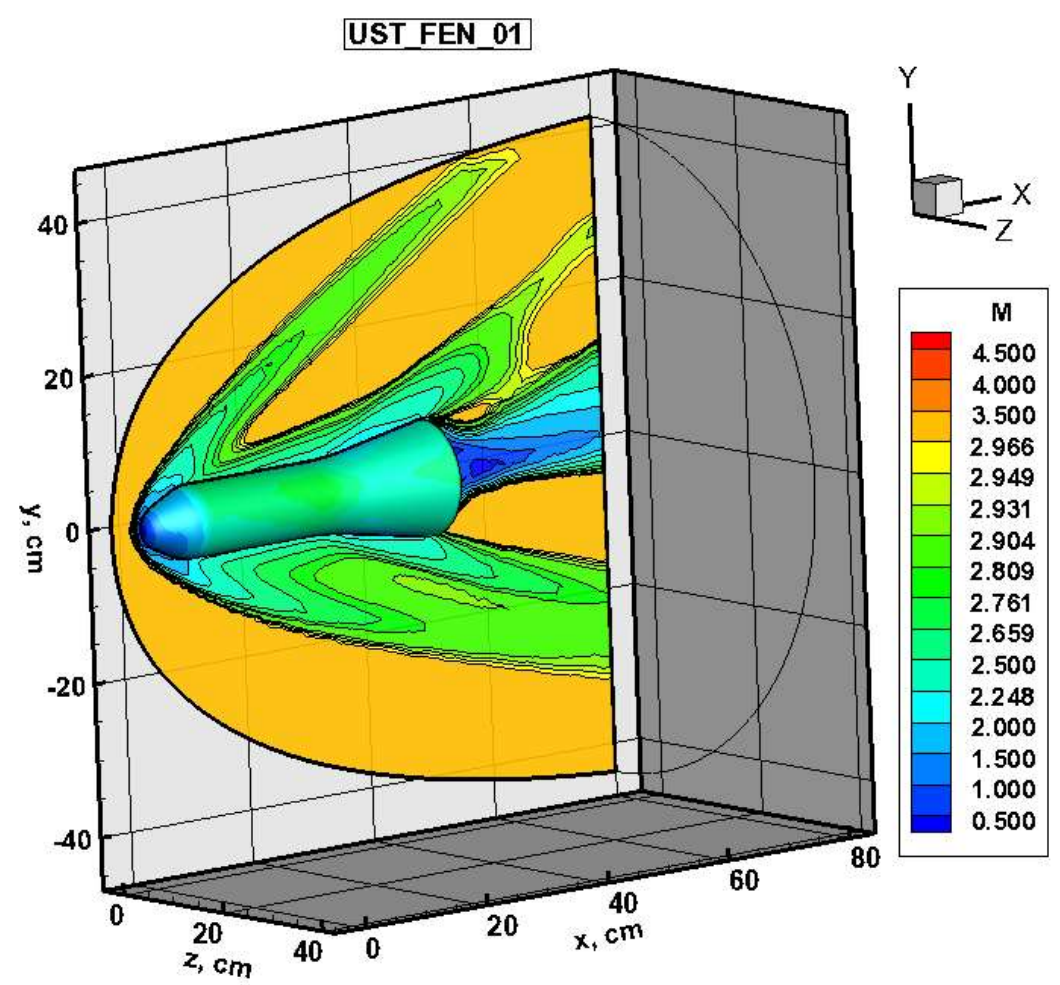

Рис. 10, $\partial$. Поле чисел Маха при $\alpha=8^{\circ}$ в плоскости симметрии $z=0$
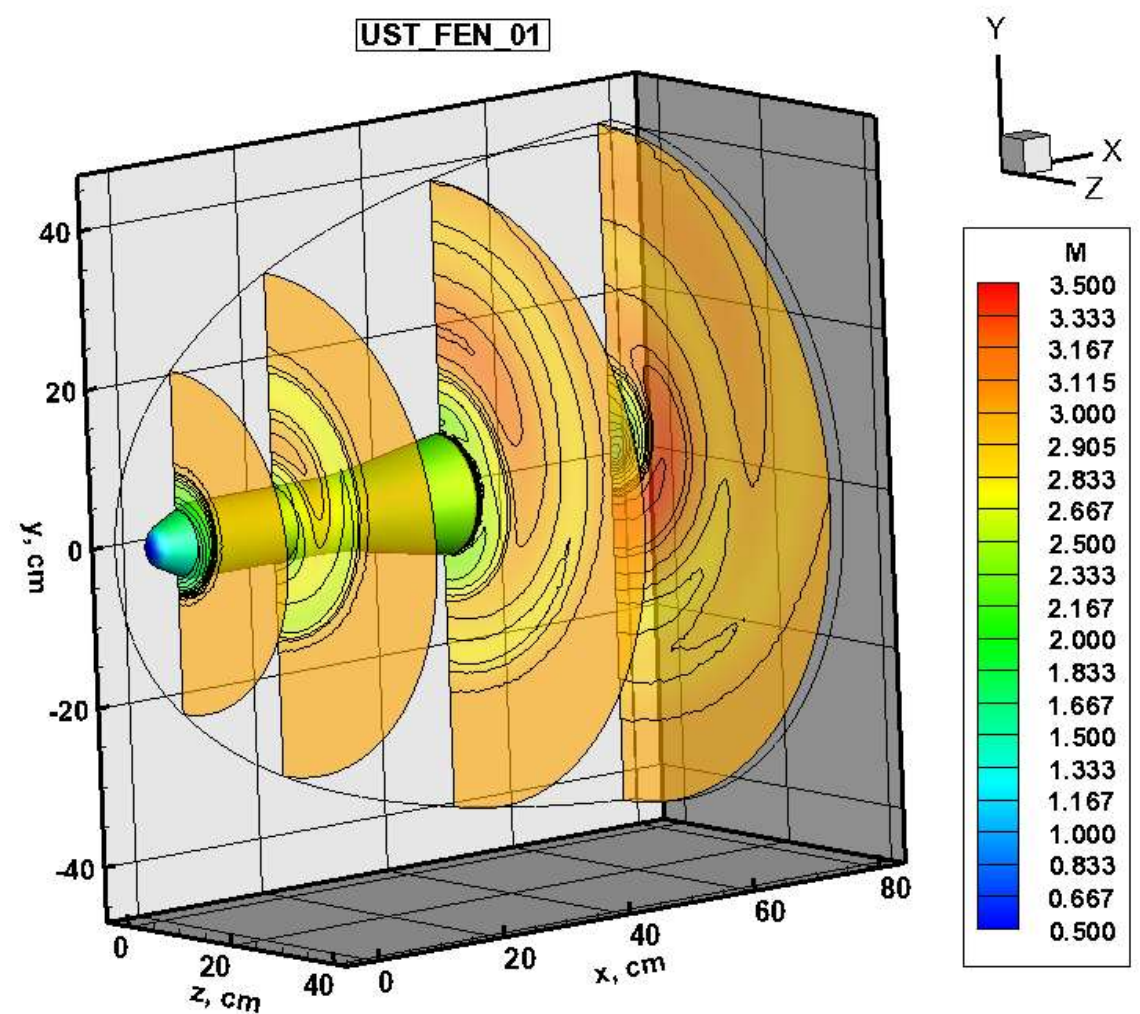

Рис. $10, e$. Поле чисел Маха при $\alpha=8^{\circ}$ в плоскостях перпендикулярных оси симметрии 


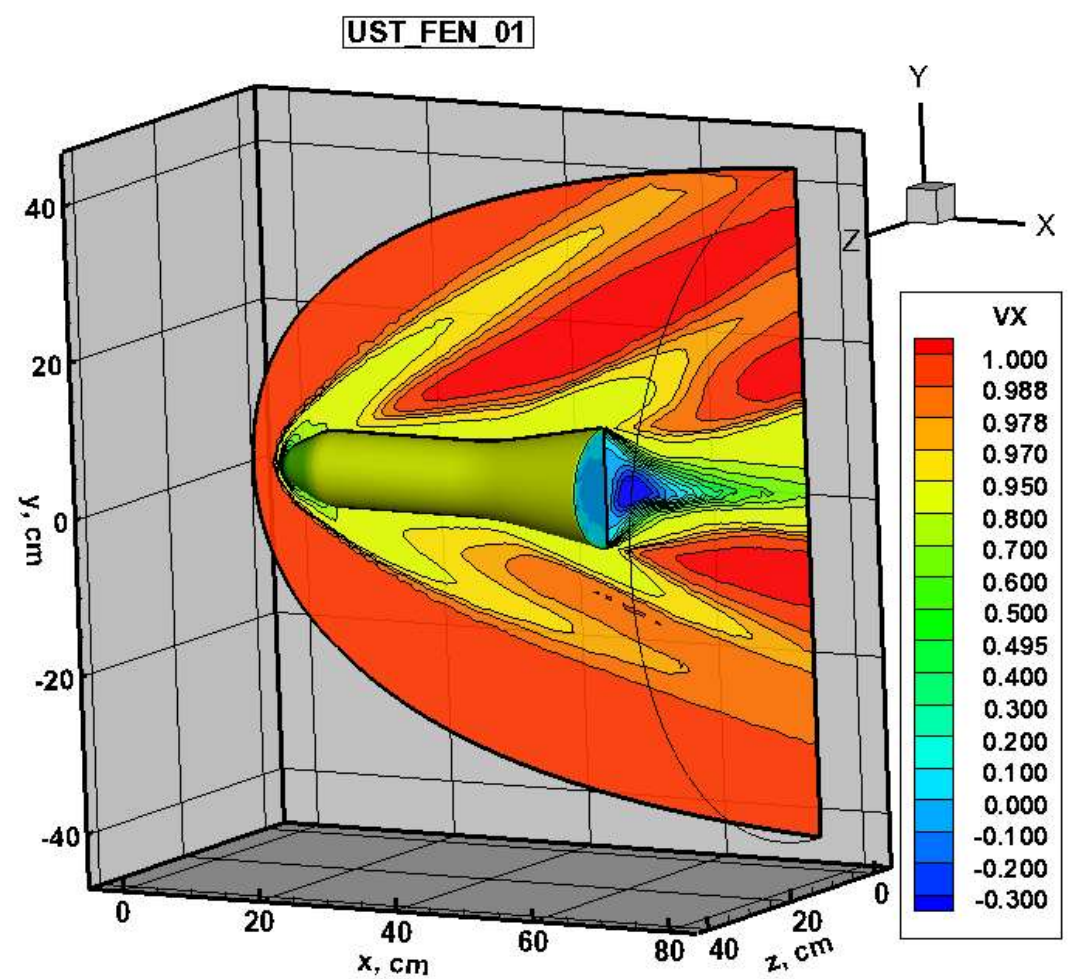

Рис. 10, ж. Поле продольной скорости $\frac{u}{u_{\infty}}$ при $\alpha=8^{\circ}$ в плоскости симметрии $z=0$

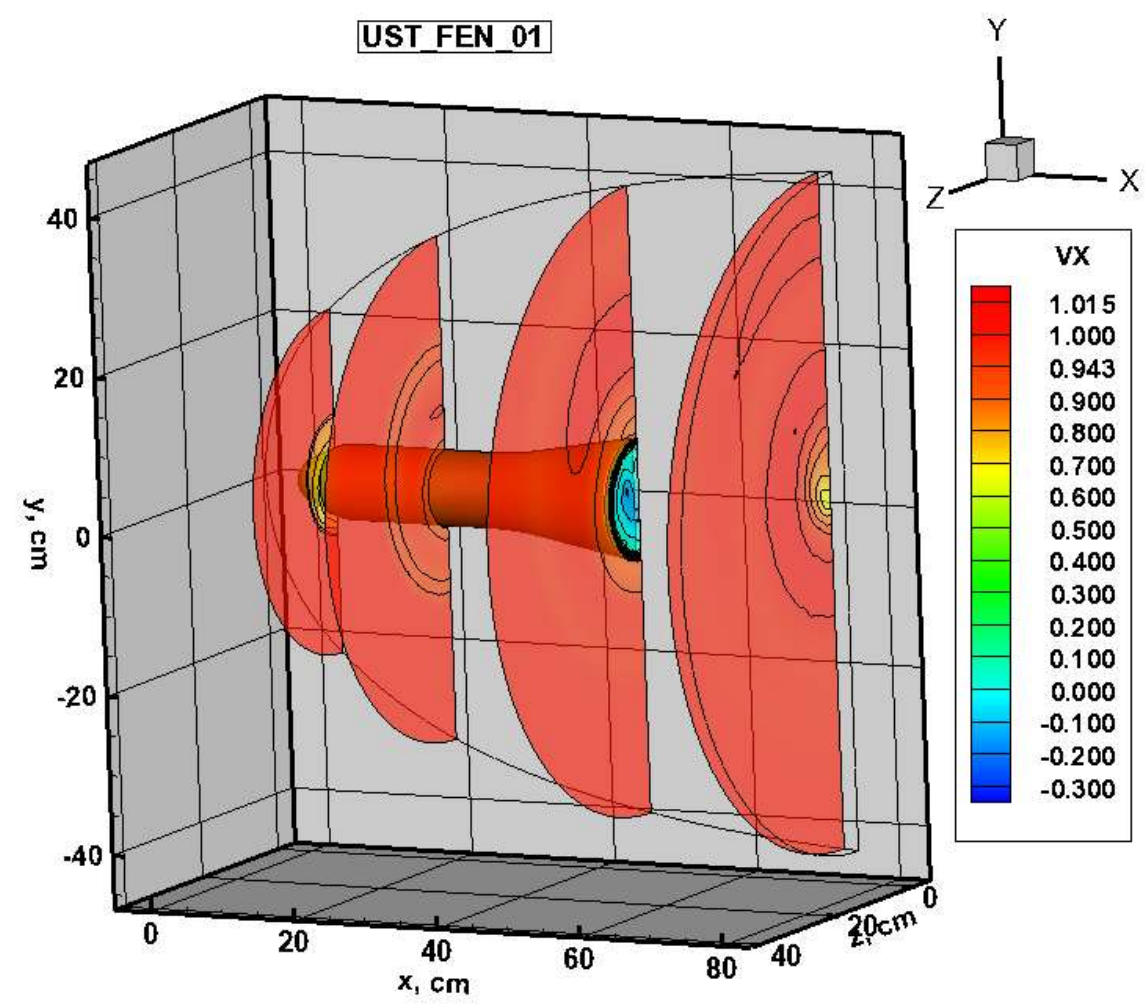

Рис. 10, з. Поле продольной скорости $\frac{u}{u_{\infty}}$ при $\alpha=8^{\circ}$ в плоскостях перпендикулярных оси симметрии 


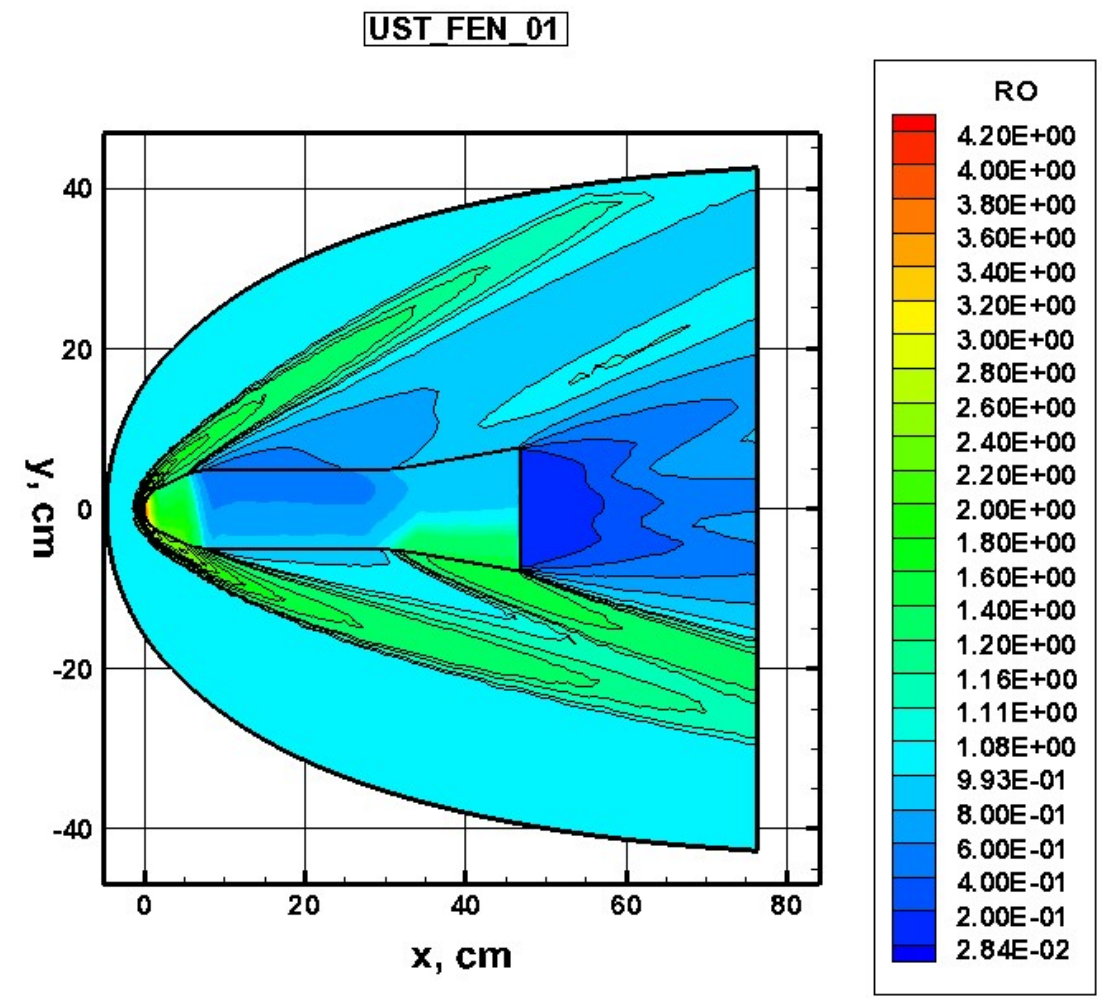

Рис. 10, $u$. Поле плотности $\frac{\rho}{\rho_{\infty}}$ при $\alpha=8^{\circ}$ в плоскости симметрии $z=0$
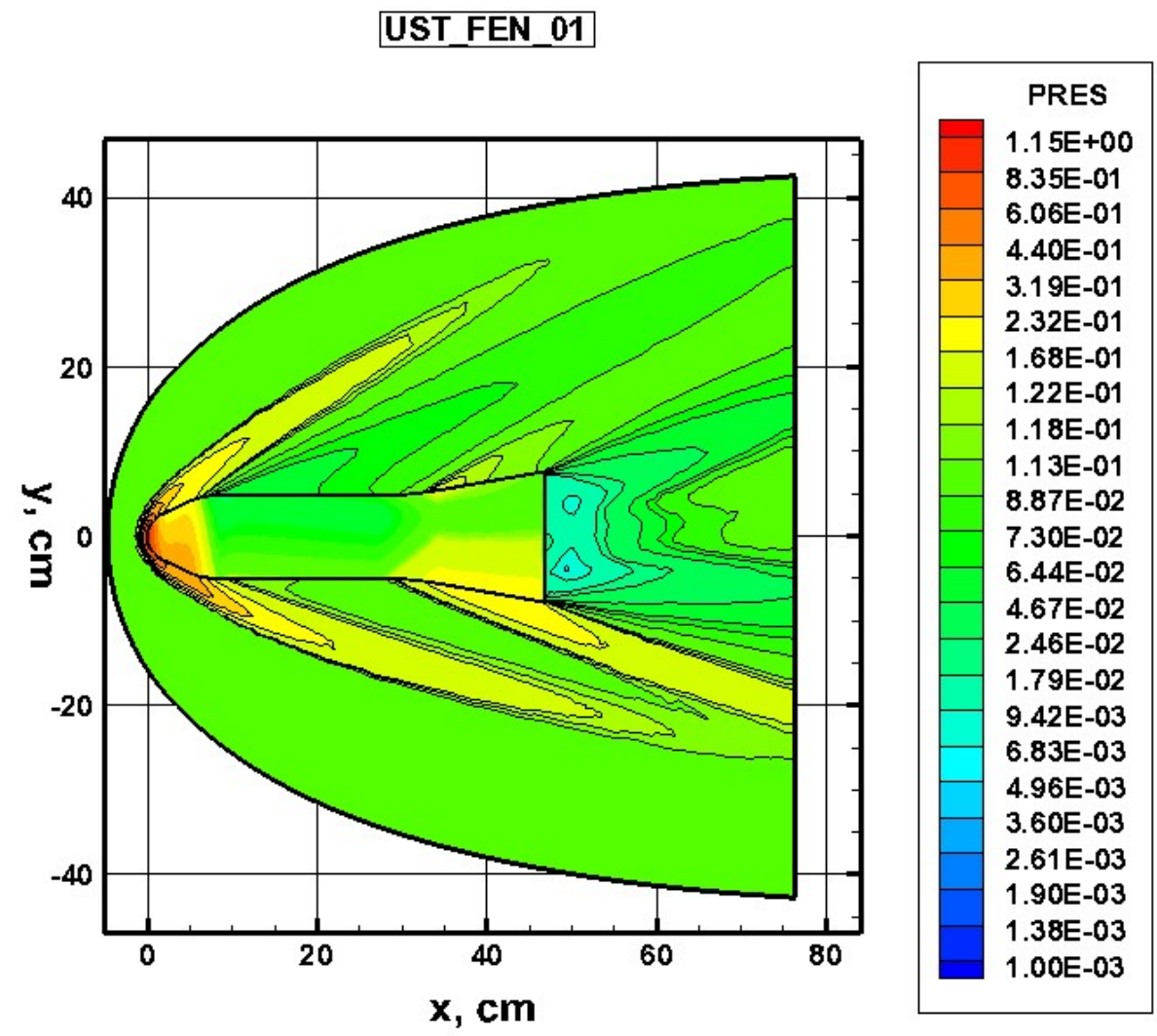

Рис. $10, \kappa$. Поле давления $\left(\mathrm{PRES}=\frac{p}{p_{0}}\right)$ при $\alpha=8^{\circ}$ в плоскости симметрии $z=0$ 
UST_FEN_01

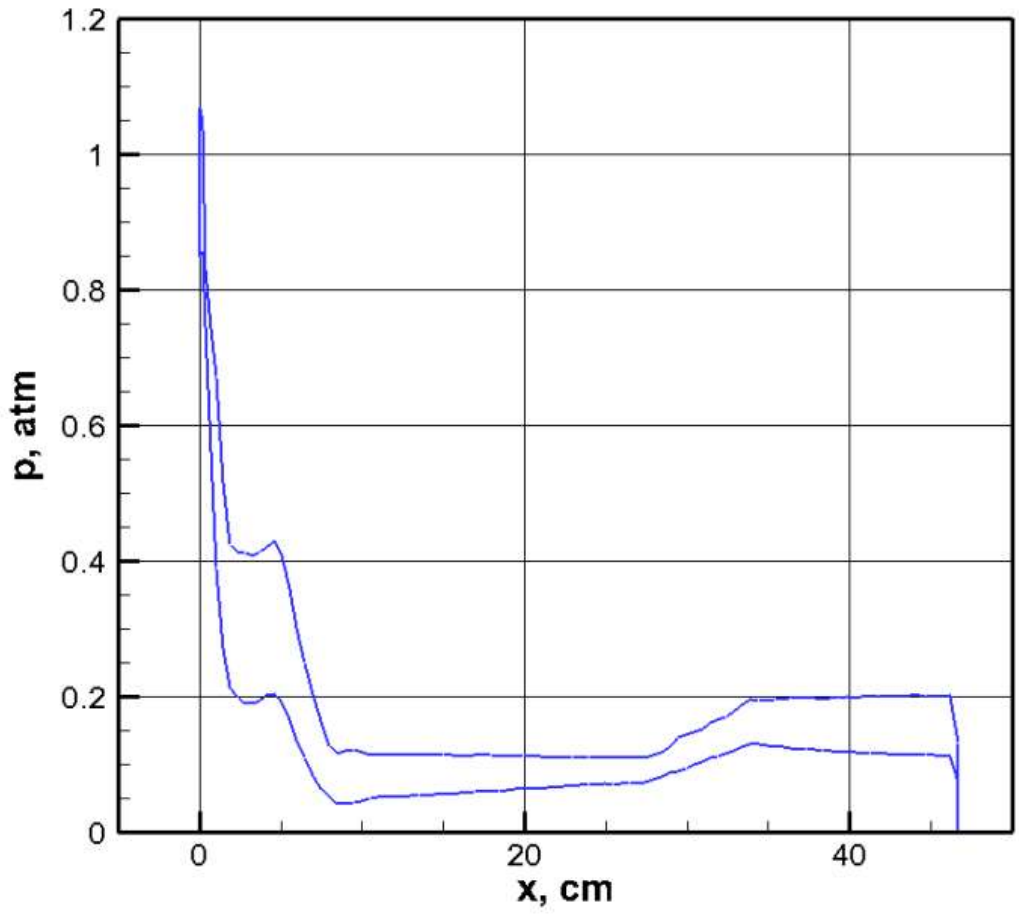

Рис. 11, $a$. Распределение давления вдоль поверхности при $\alpha=8^{\circ}$ в плоскости симметрии $z=0$

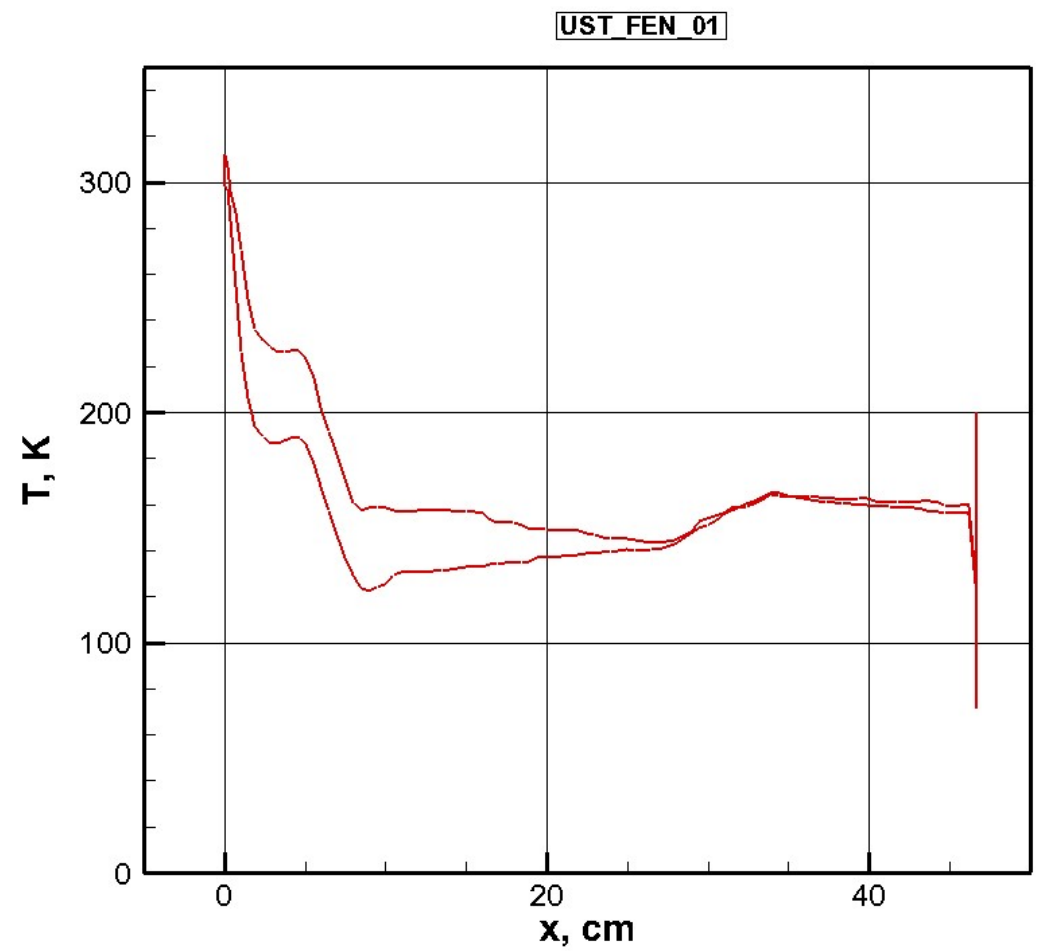

Рис. 11, б. Распределение температуры вдоль поверхности при $\alpha=8^{\circ}$ в плоскости симметрии $z=0$ 


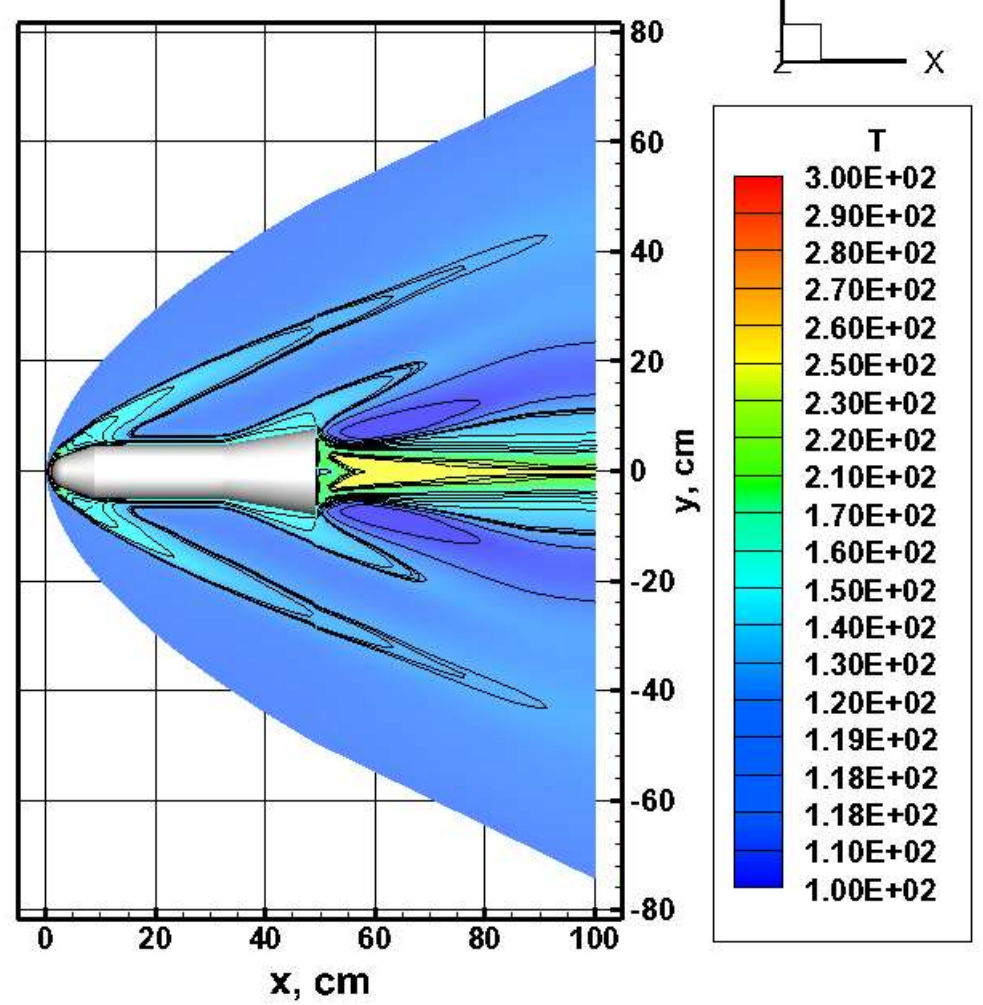

Рис. $12, a$. Поле температуры в К при $\alpha=0$ в плоскости симметрии $z=0$

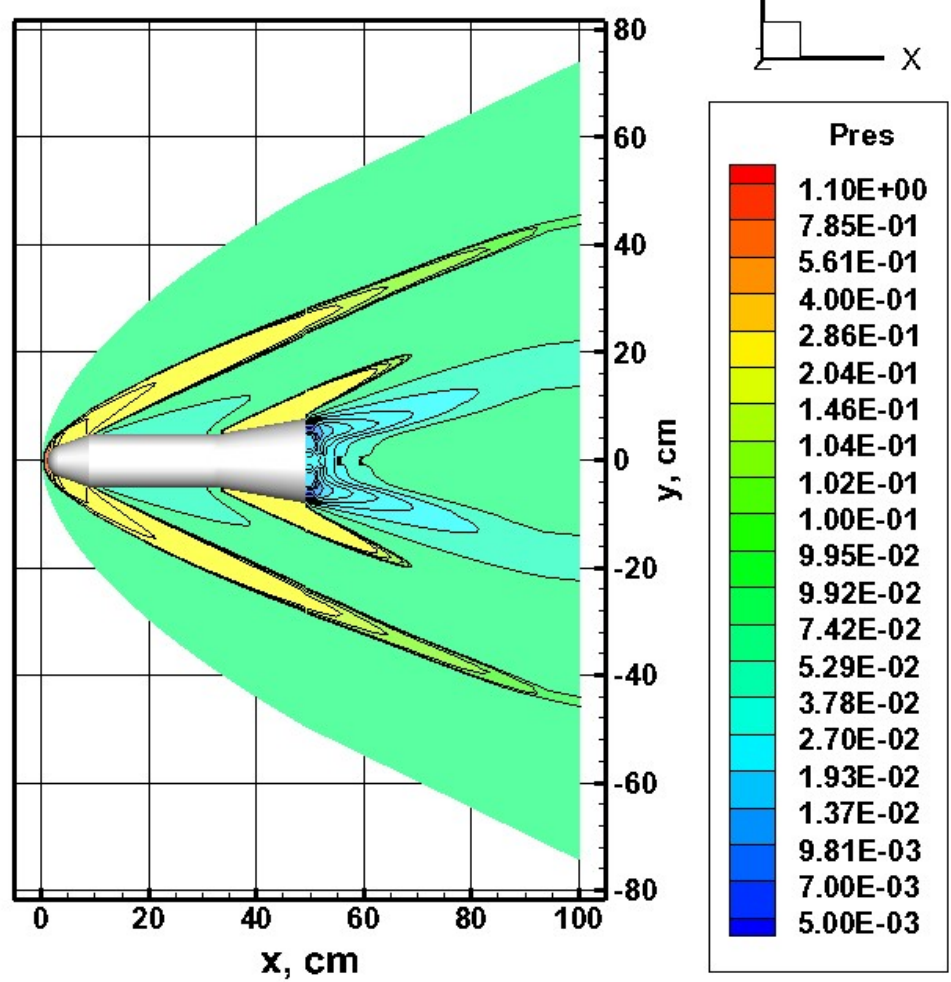

Рис. 12, б. Поле давления ( $\mathrm{PRES}=\frac{p}{p_{0}}$ ) при $\alpha=0$ в плоскости симметрии $z=0$ 


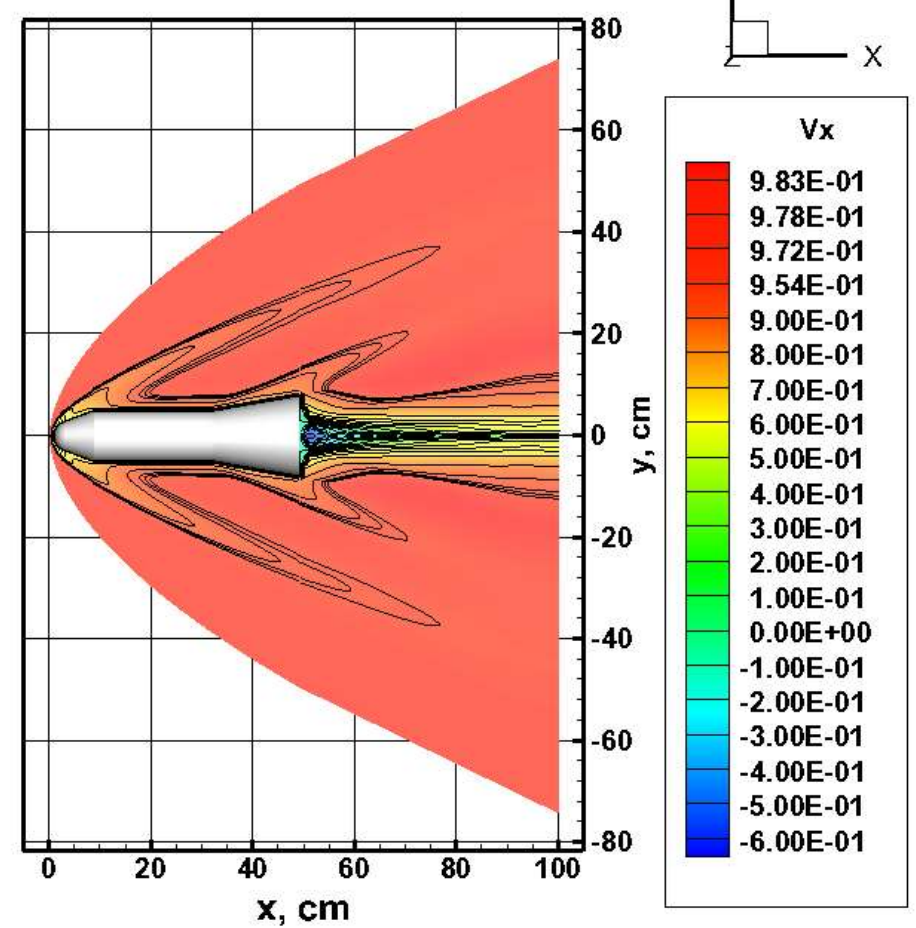

Рис. 12, в. Поле продольной скорости $\frac{u}{u_{\infty}}$ при $\alpha=0$ в плоскости симметрии $z=0$

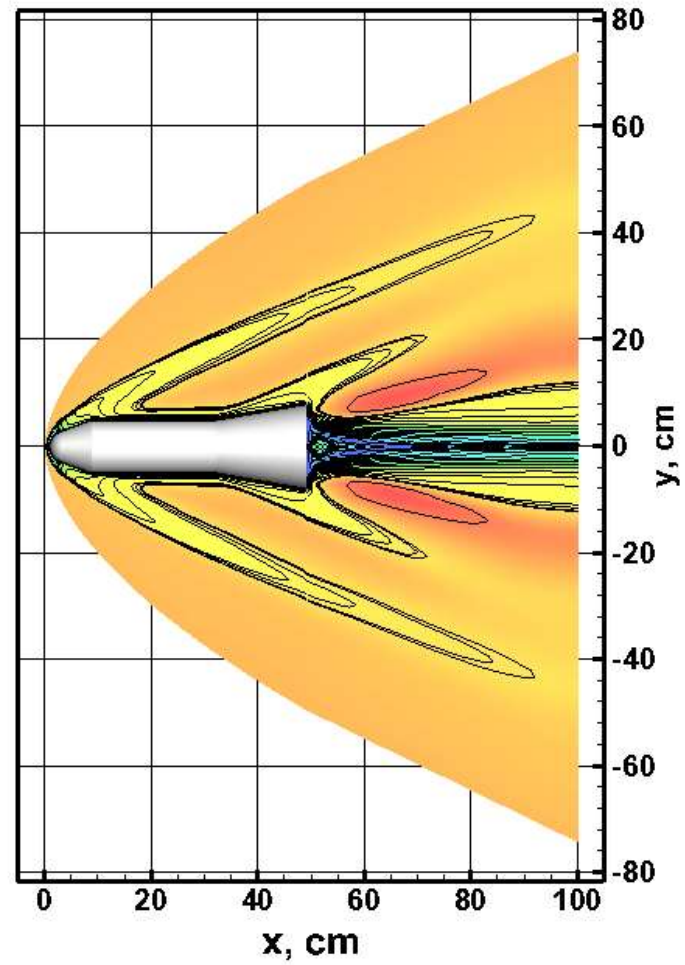

Y

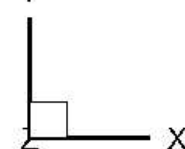

M

$3.20 \mathrm{E}+00$

$2.87 \mathrm{E}+00$

$2.86 \mathrm{E}+00$

$2.83 \mathrm{E}+00$

$2.80 \mathrm{E}+00$

$2.60 \mathrm{E}+00$

$2.40 \mathrm{E}+00$

$2.20 \mathrm{E}+00$

$2.00 \mathrm{E}+00$

1. $80 \mathrm{E}+00$

$1.60 \mathrm{E}+00$

1. $40 \mathrm{E}+00$

1. $20 \mathrm{E}+00$

1. $00 \mathrm{E}+00$

8.00E-01

6.00E-01

4.00E-01

2.00E-01

Рис. 12 , г. Поле чисел Маха при $\alpha=0$ в плоскости симметрии $z=0$ 


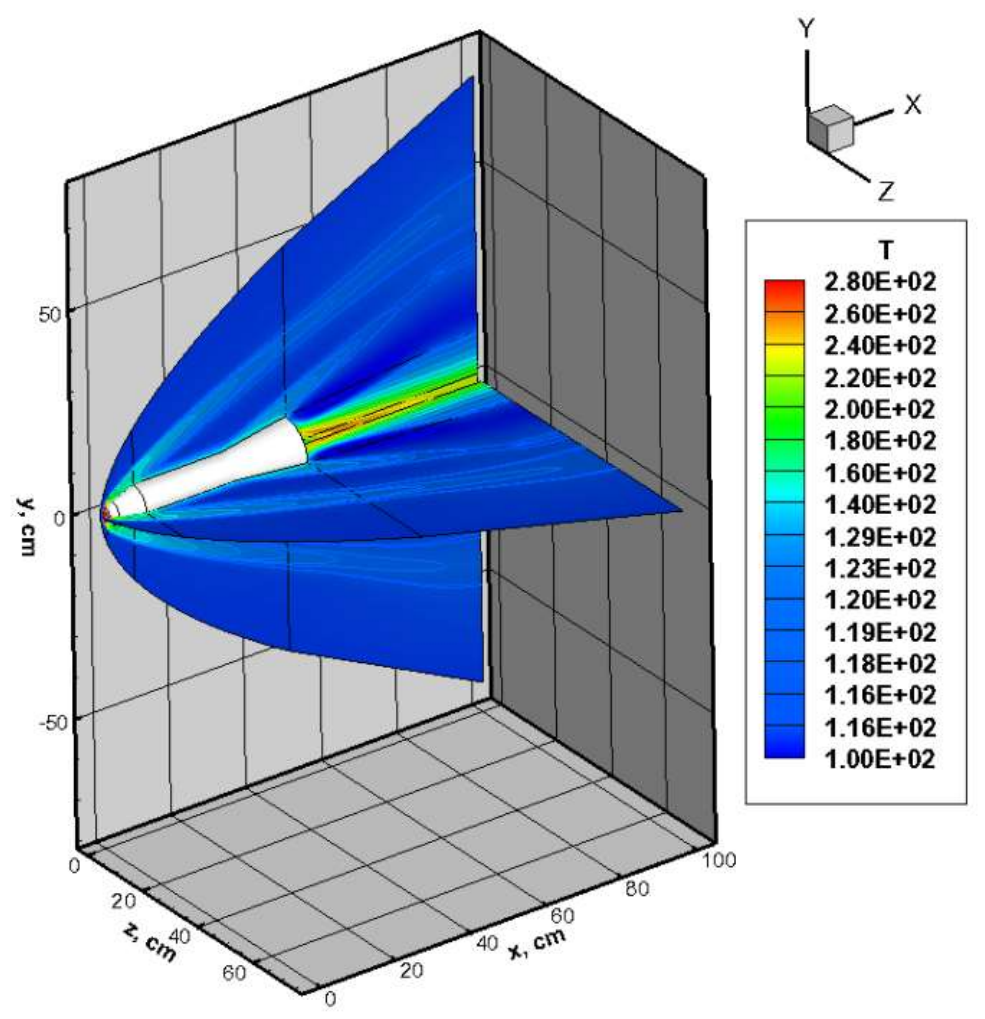

Рис. 12, д. Поле температуры в К при $\alpha=0$ в плоскостях $y=0$ и $z=0$

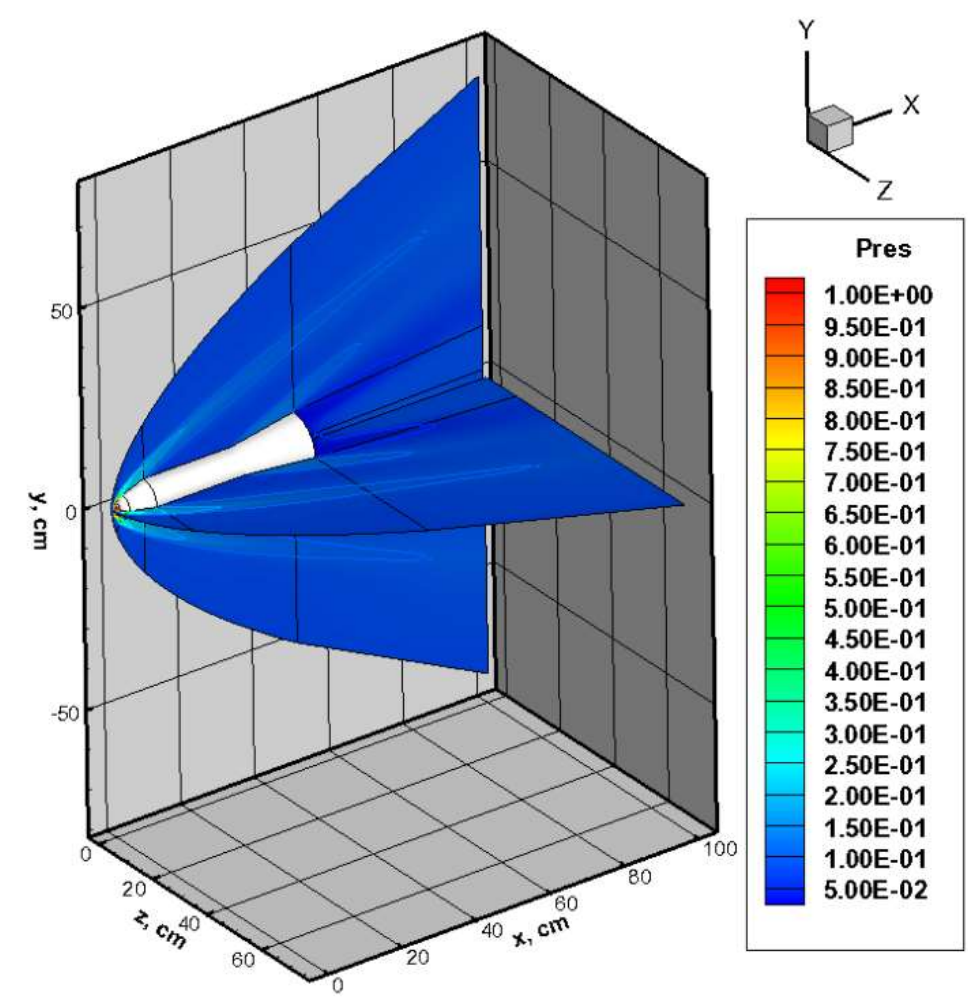

Рис. 12, е. Поле давления ( $\left.\mathrm{PRES}=\frac{p}{p_{0}}\right)$ при $\alpha=0$ в плоскостях $y=0$ и $z=0$ 


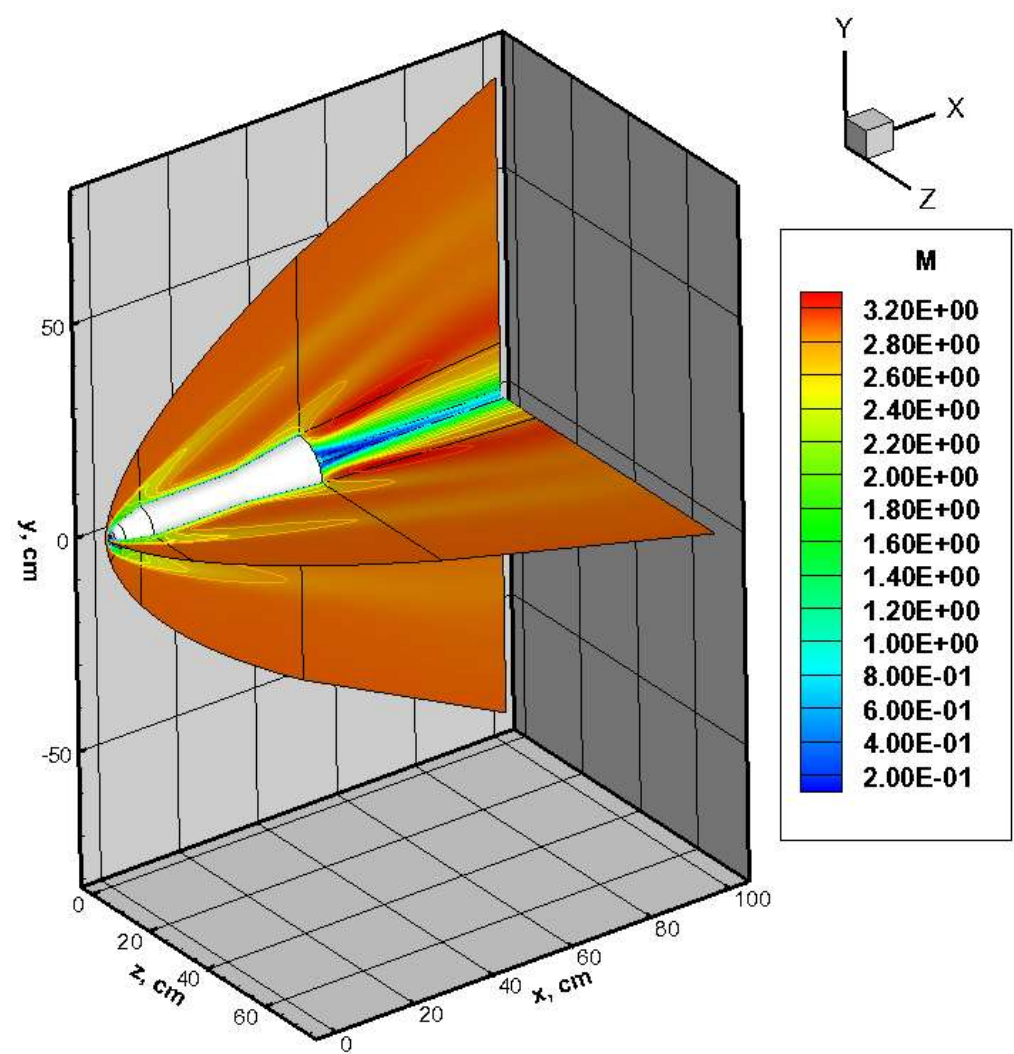

Рис. $12, ж$. Поле чисел Маха при $\alpha=0$ в плоскостях $y=0$ и $z=0$

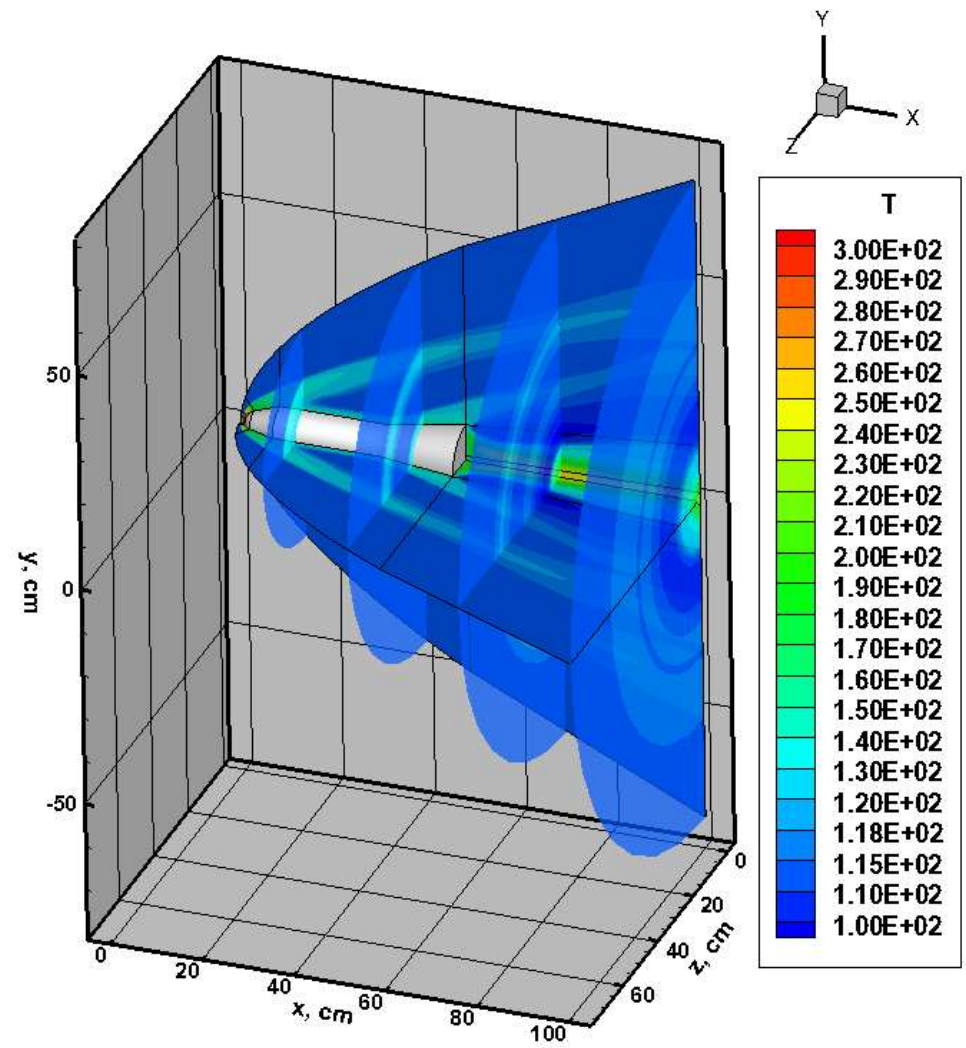

Рис. 12 , з. Поле температуры в $\mathrm{K}$ при $\alpha=0$ в плоскостях перпендикулярных оси симметрии 


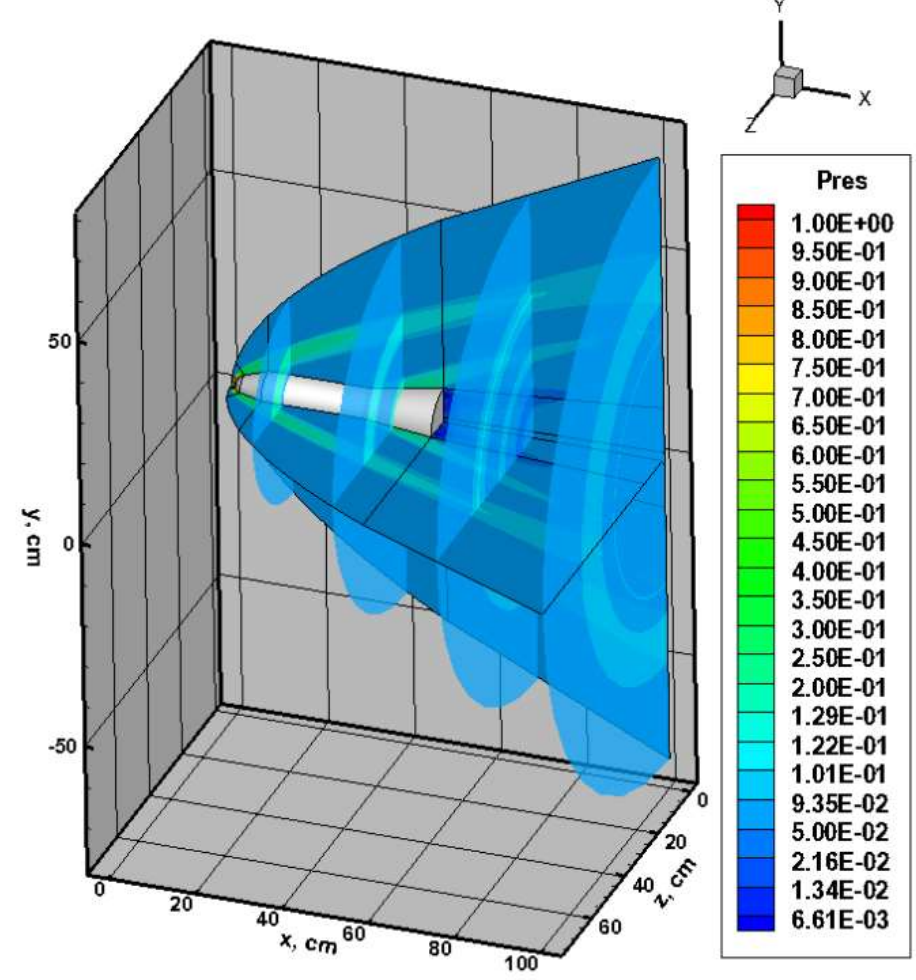

Рис. 12, $u$. Поле давления ( $\mathrm{PRES}=\frac{p}{p_{0}}$ ) при $\alpha=0$ в плоскостях перпендикулярных оси симметрии

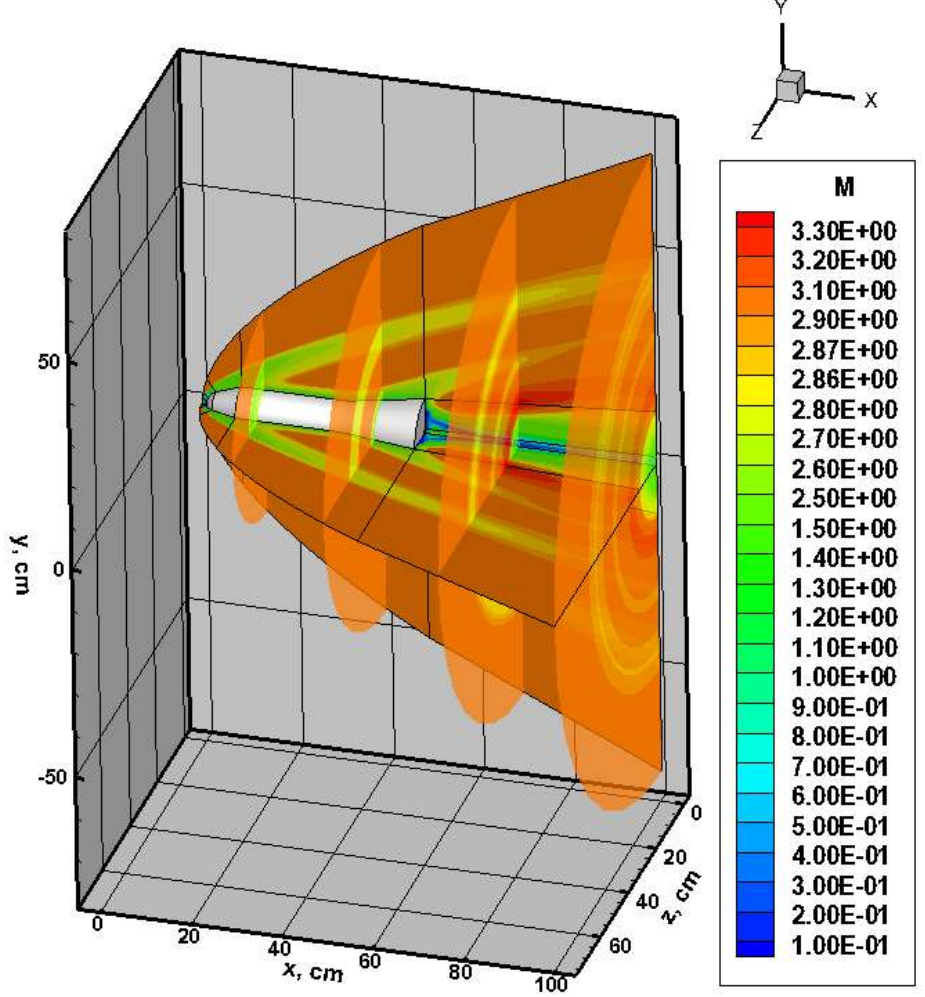

Рис. $12, \kappa$. Поле чисел Маха при $\alpha=0$ в плоскостях перпендикулярных оси симметрии 


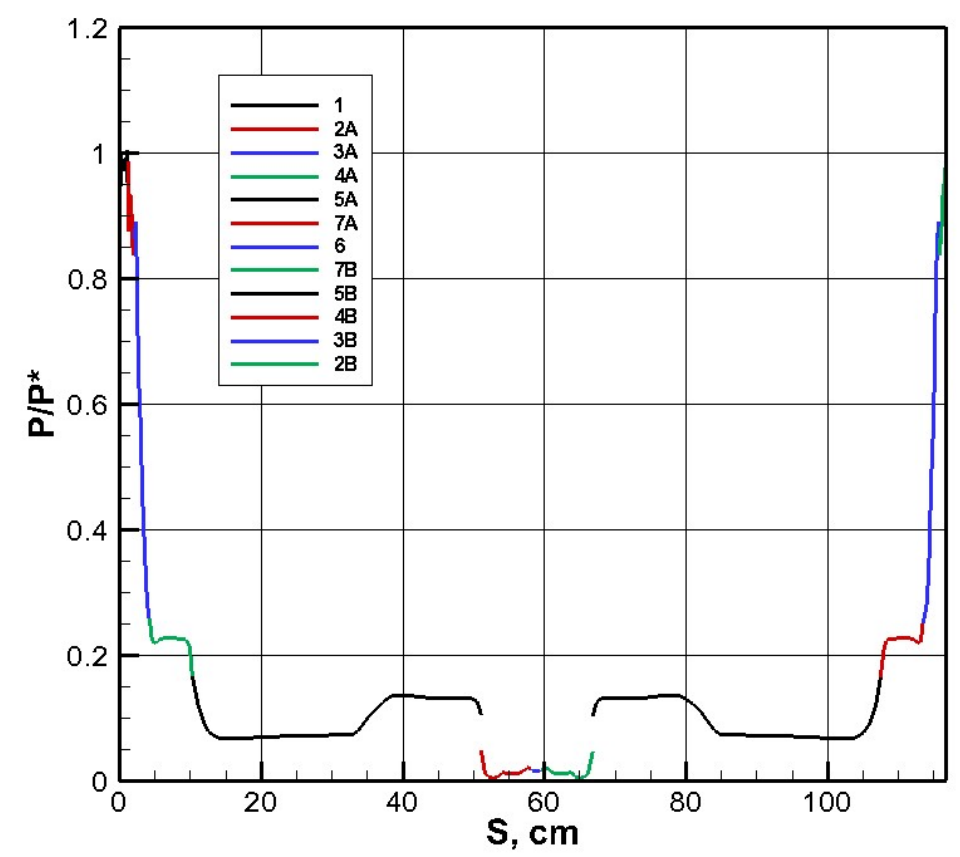

Рис. 13. Распределение давления вдоль поверхности при $\alpha=0$ в плоскости симметрии $z=0$
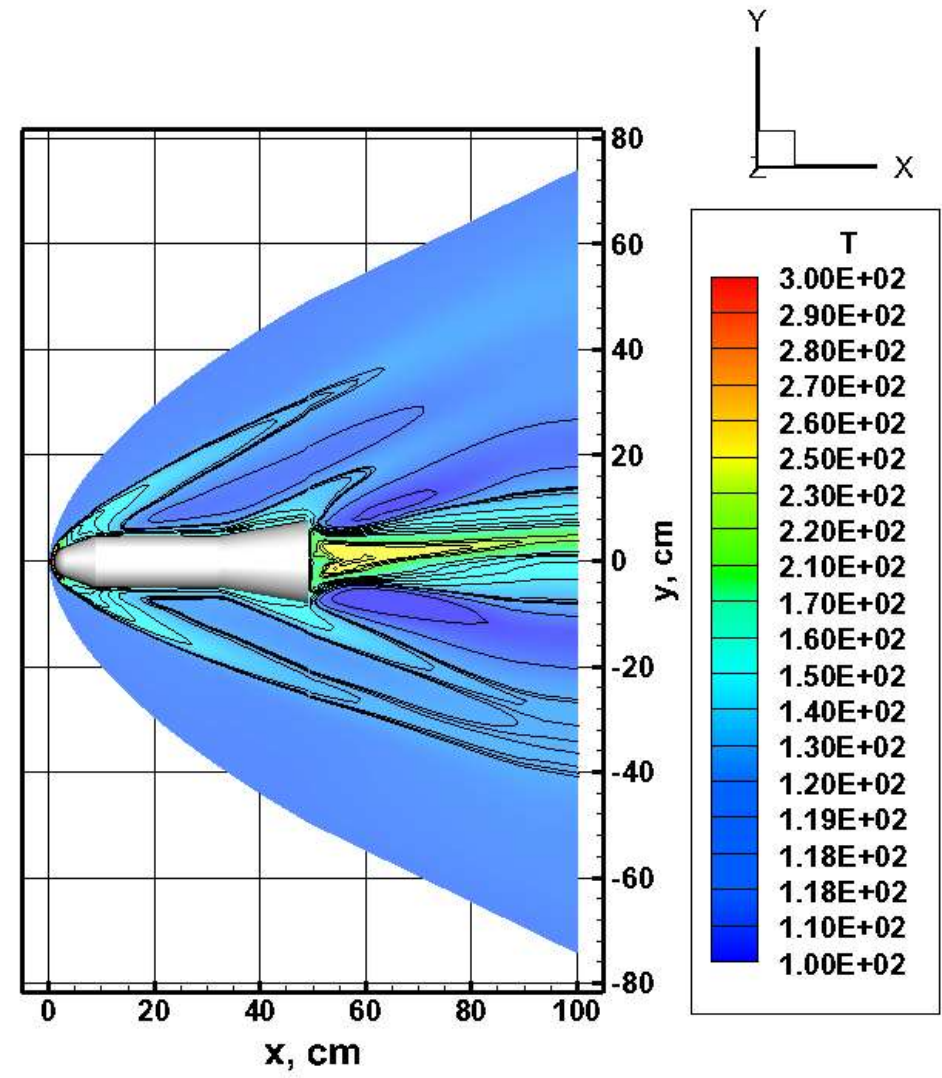

Рис.14, $a$. Поле температуры в К при $\alpha=4^{\circ}$ в плоскости симметрии $z=0$ 


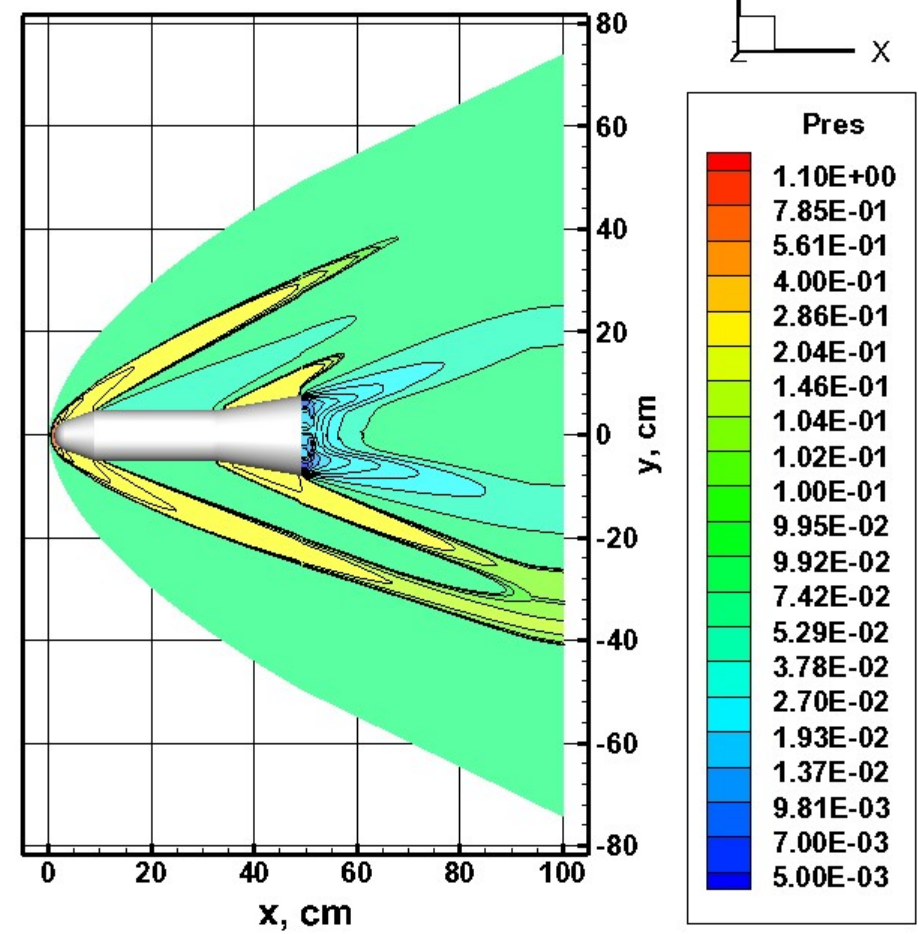

Рис. 14, б. Поле давления ( $\left.\mathrm{PRES}=\frac{p}{p_{0}}\right)$ при $\alpha=4^{\circ}$ в плоскости симметрии $z=0$
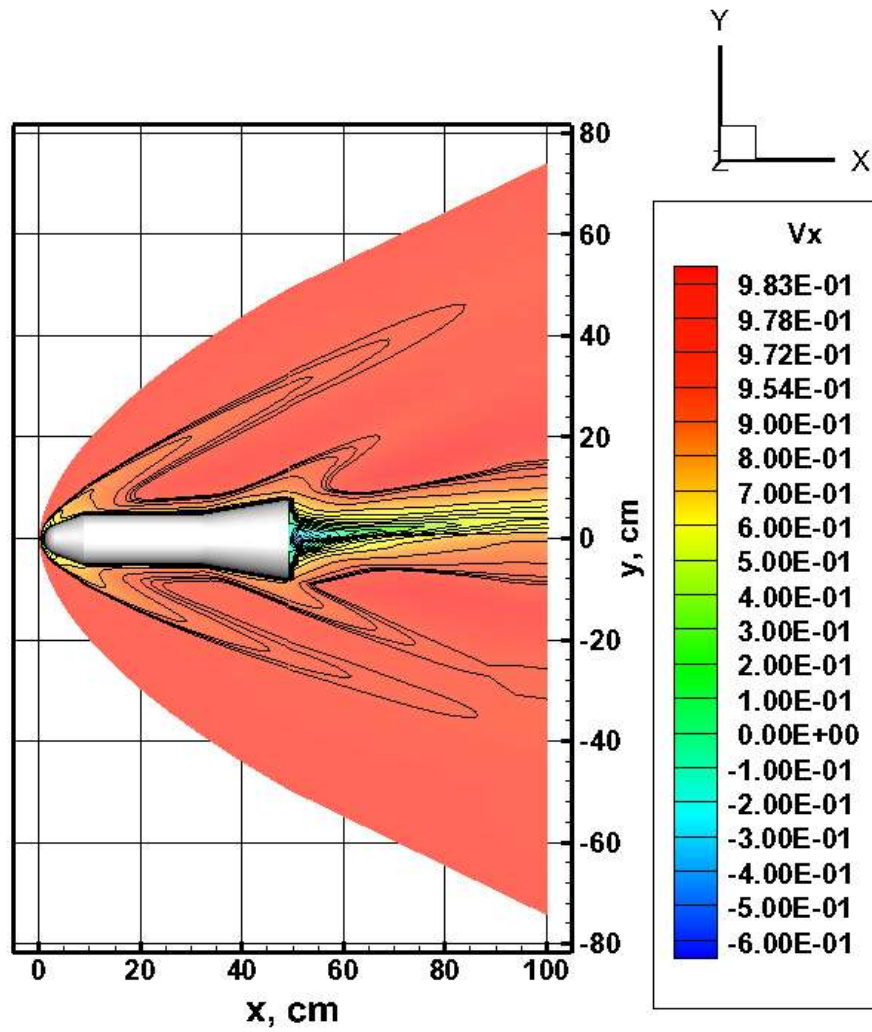

9.83E-01

9.78E-01

9.72E-01

9.54E-01

9.00E-01

8.00E-01

7.00E-01

6.00E-01

5.00E-01

4.00E-01

3.00E-01

2.00E-01

$1.00 \mathrm{E}-01$

$0.00 \mathrm{E}+00$

$-1.00 \mathrm{E}-01$

-2.00E-01

$-3.00 \mathrm{E}-01$

$-4.00 \mathrm{E}-01$

$-5.00 \mathrm{E}-01$

$-6.00 \mathrm{E}-01$

Рис. 14, в. Поле продольной скорости $\frac{u}{u_{\infty}}$ при $\alpha=4^{\circ}$ в плоскости симметрии $z=0$ 

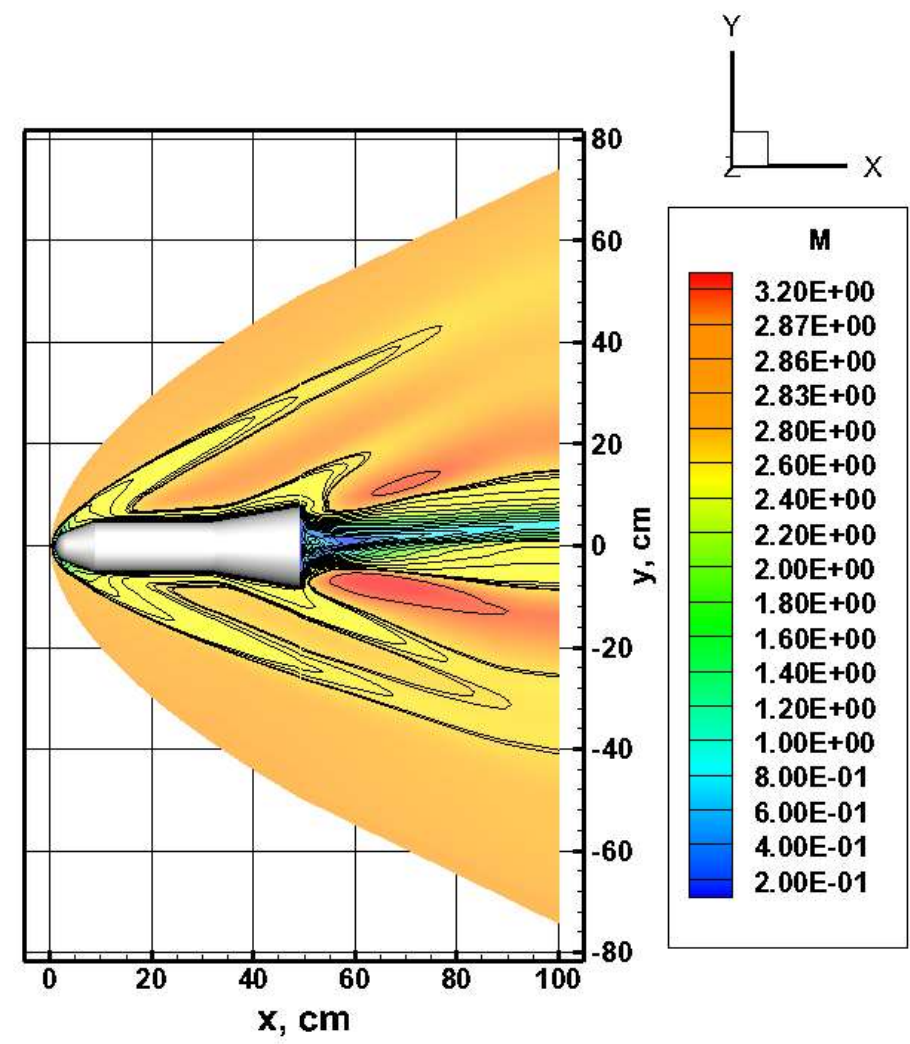

Рис. 14, 2. Поле чисел Маха при $\alpha=4^{\circ}$ в плоскости симметрии $z=0$

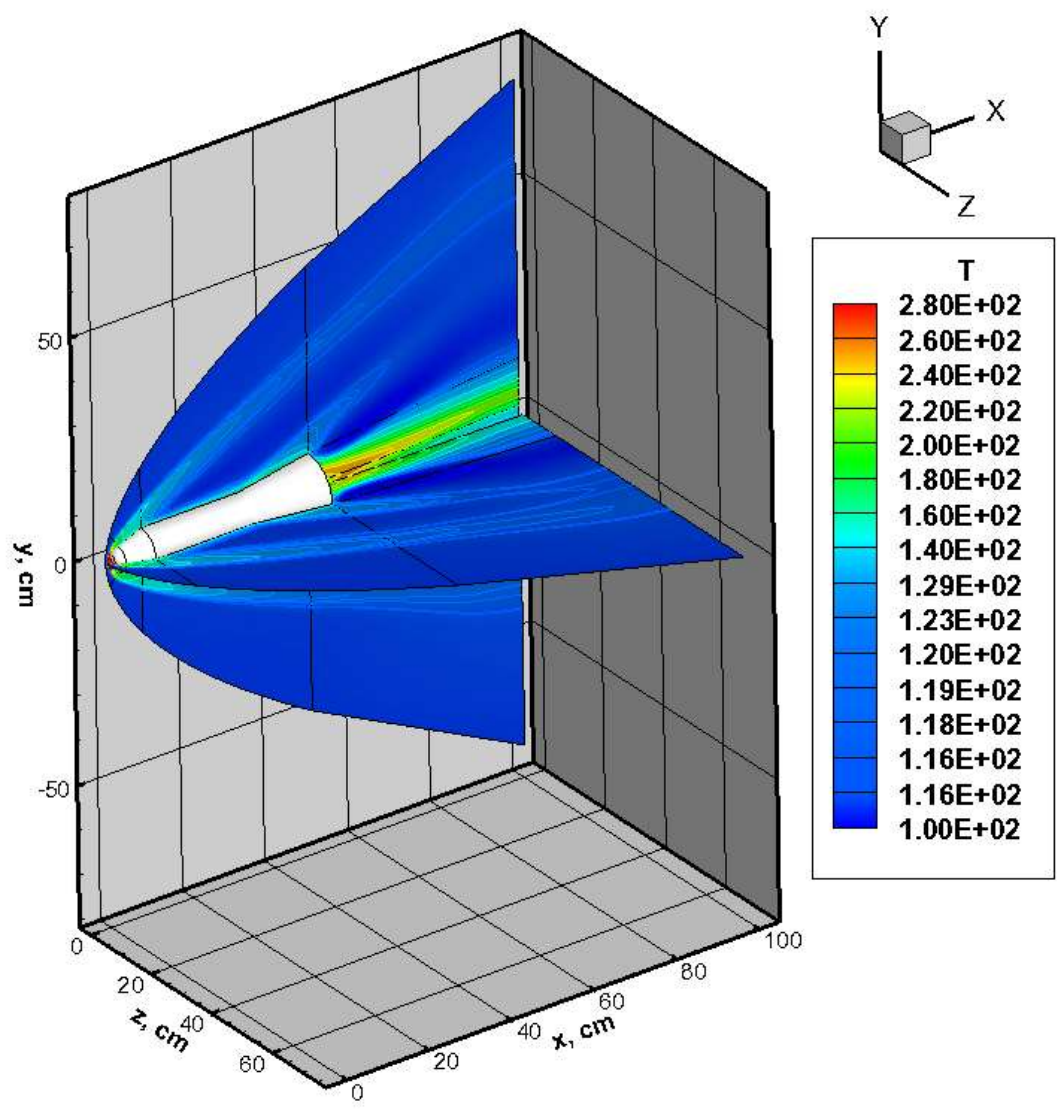

Рис. 14, $\partial$. Поле температуры в $\mathrm{K}$ при $\alpha=4^{\circ}$ в плоскостях $y=0$ и $z=0$ 


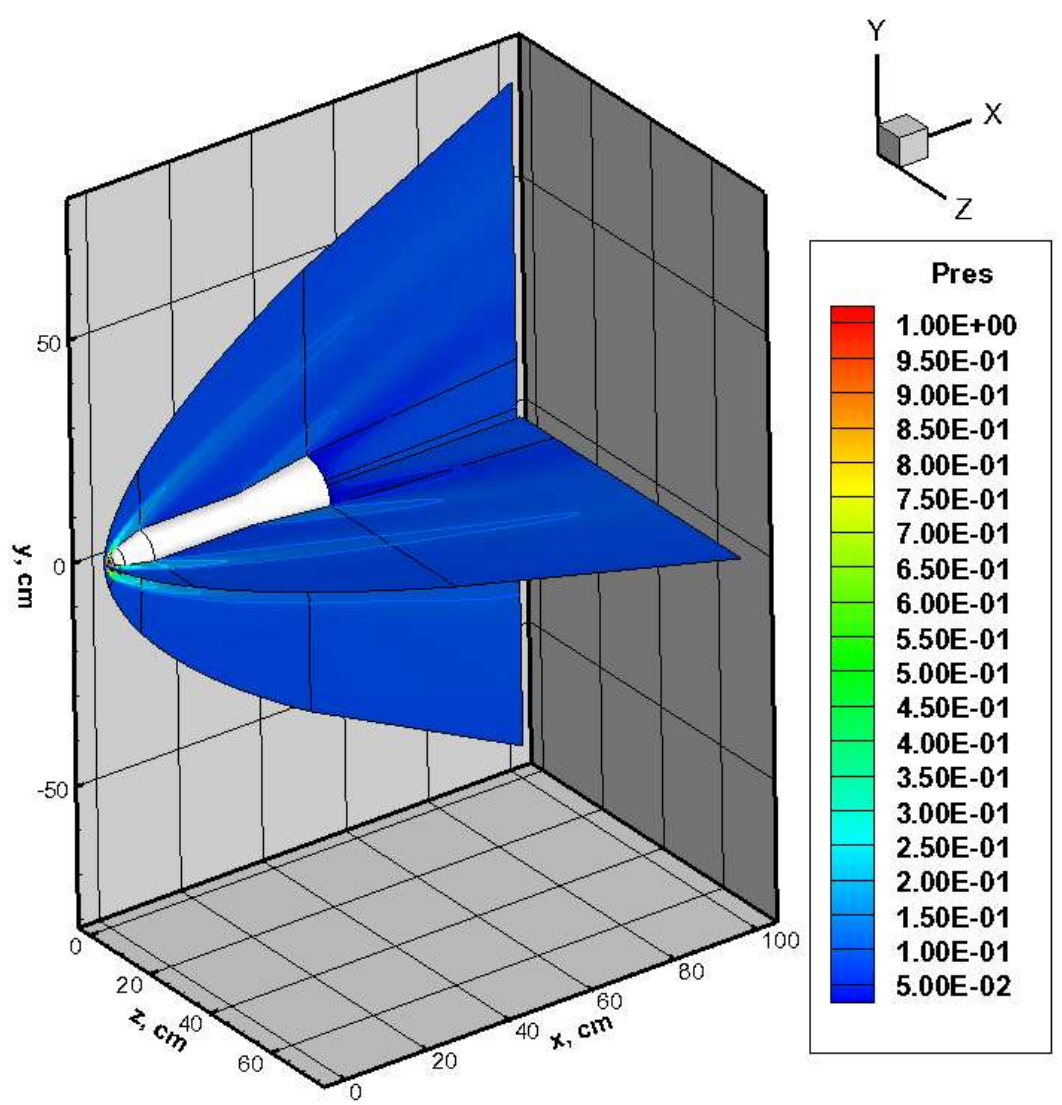

Рис. 14, е. Поле давления ( $\left.\mathrm{PRES}=\frac{p}{p_{0}}\right)$ при $\alpha=4^{\circ}$ в плоскостях $y=0$ и $z=0$

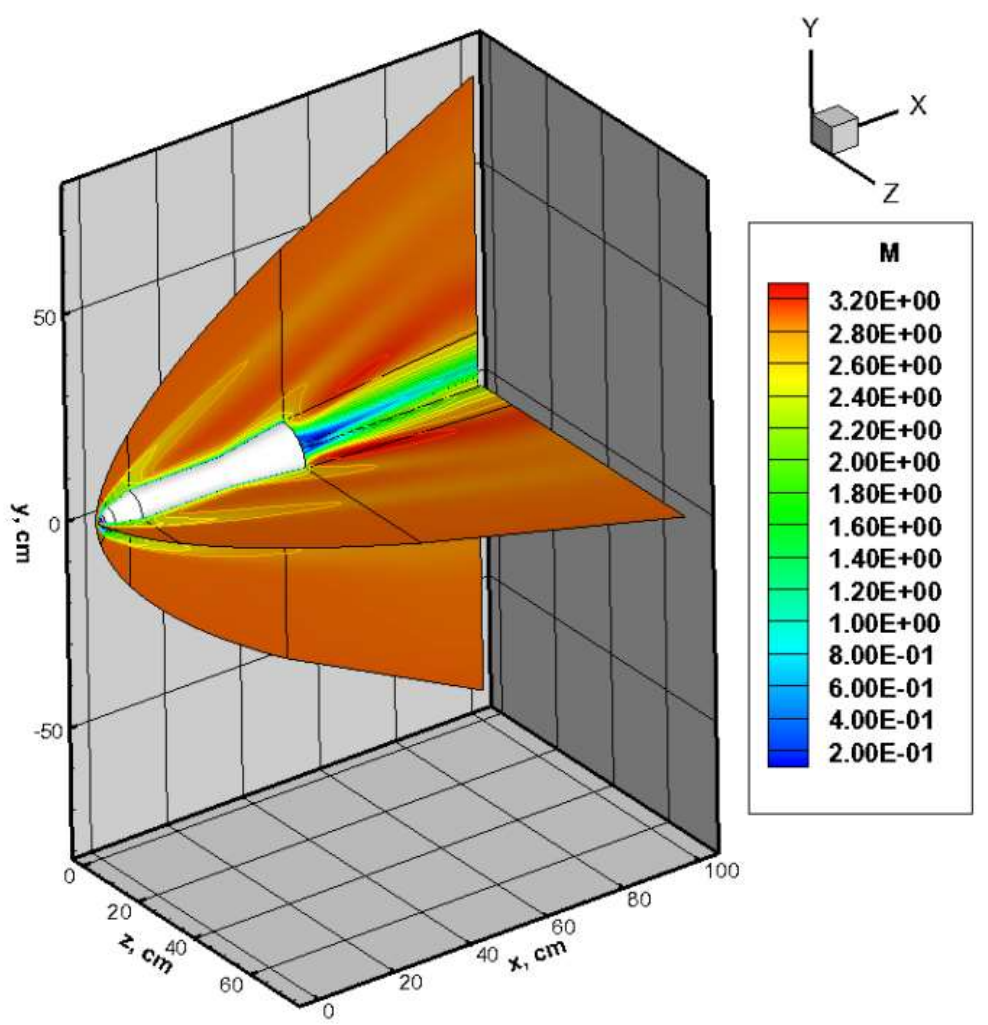

Рис. $14, ж$. Поле чисел Маха при $\alpha=4^{\circ}$ в плоскостях $y=0$ и $z=0$ 


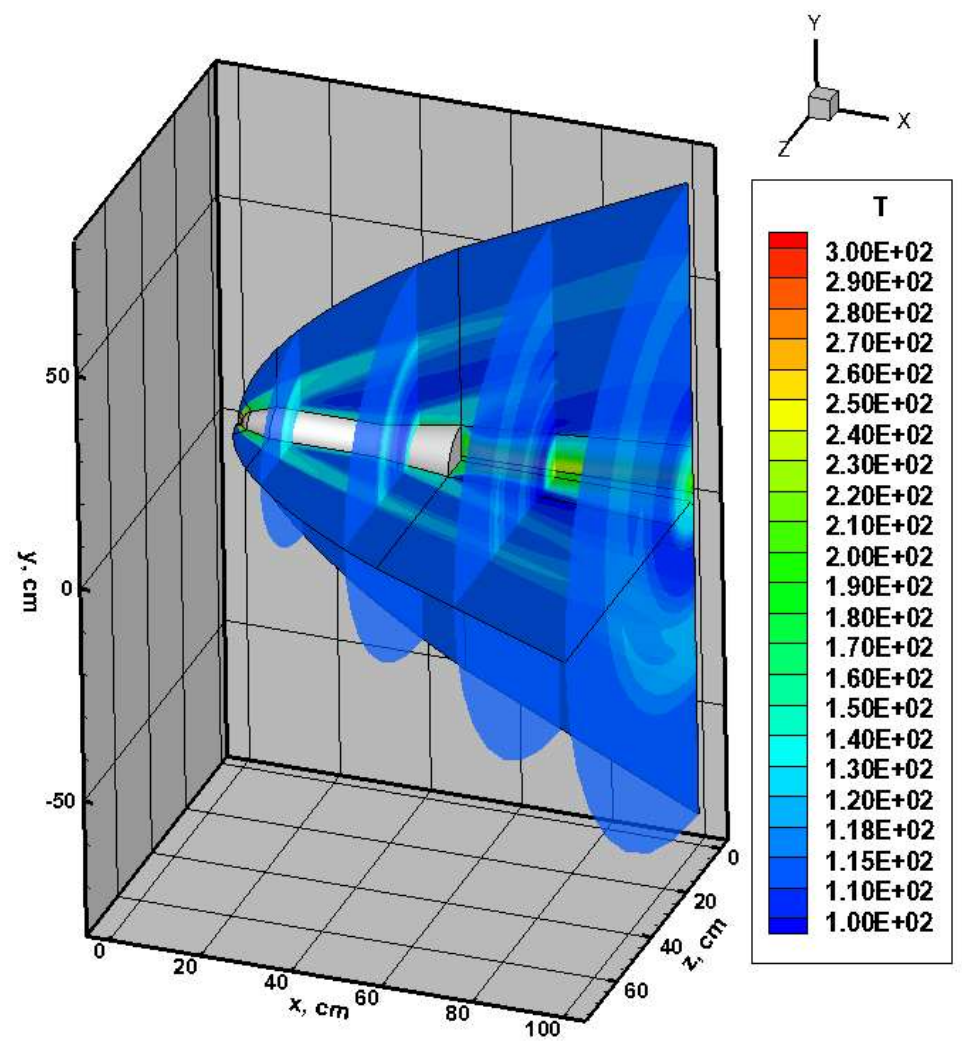

Рис. 14 , з. Поле температуры в $\mathrm{K}$ при $\alpha=4^{\circ}$ в плоскостях перпендикулярных оси симметрии

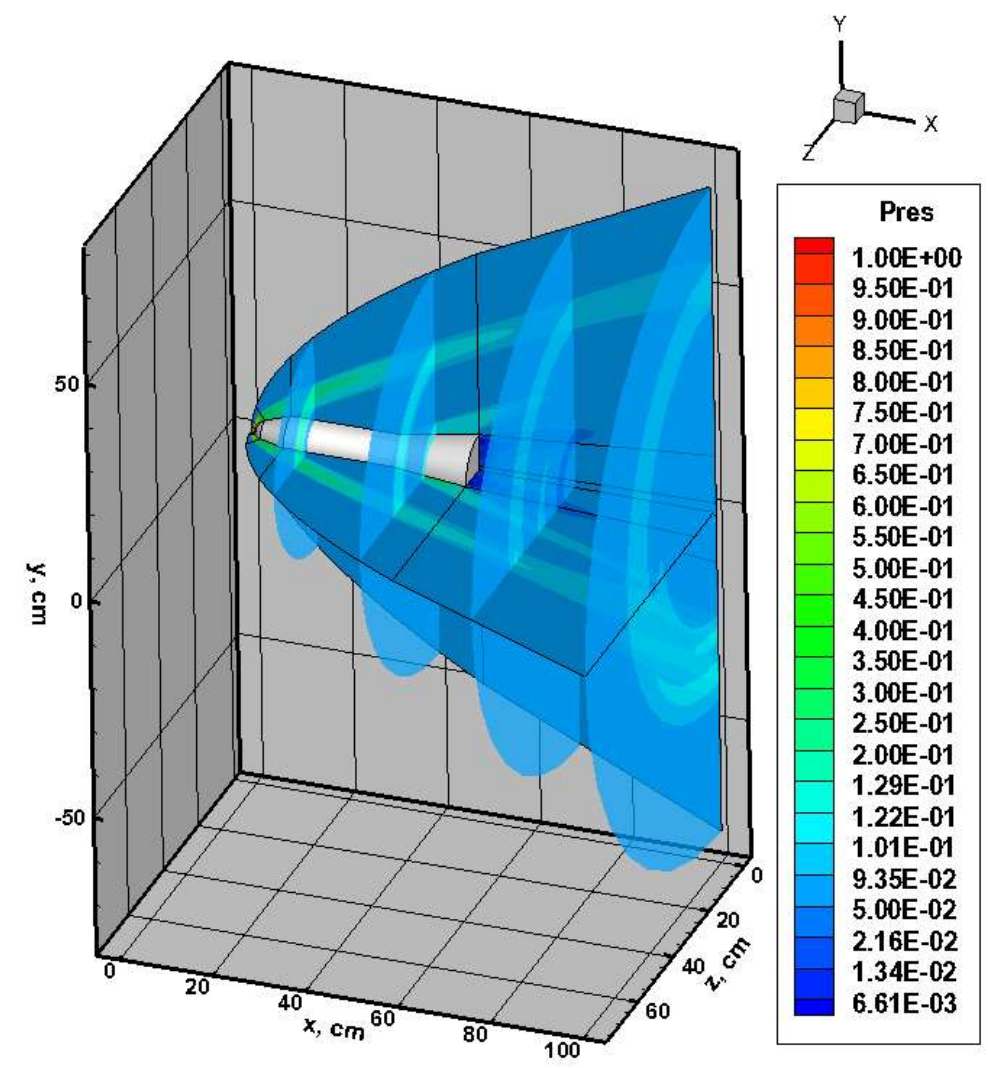

Рис. $14, u$. Поле давления ( $\mathrm{PRES}=\frac{p}{p_{0}}$ ) при $\alpha=4^{\circ}$ в плоскостях перпендикулярных оси симметрии 

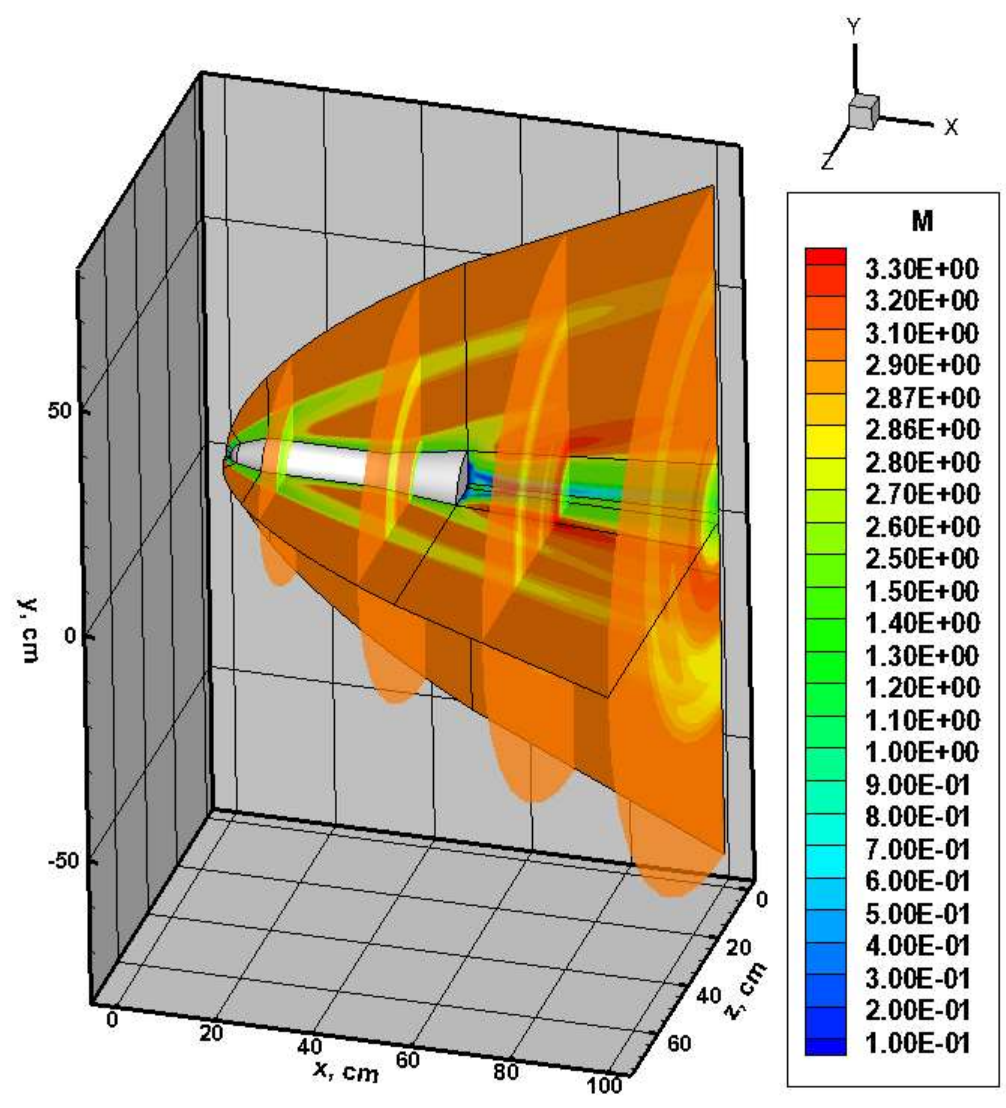

Рис. $14, \kappa$. Поле чисел Маха при $\alpha=4^{\circ}$ в плоскостях перпендикулярных оси симметрии

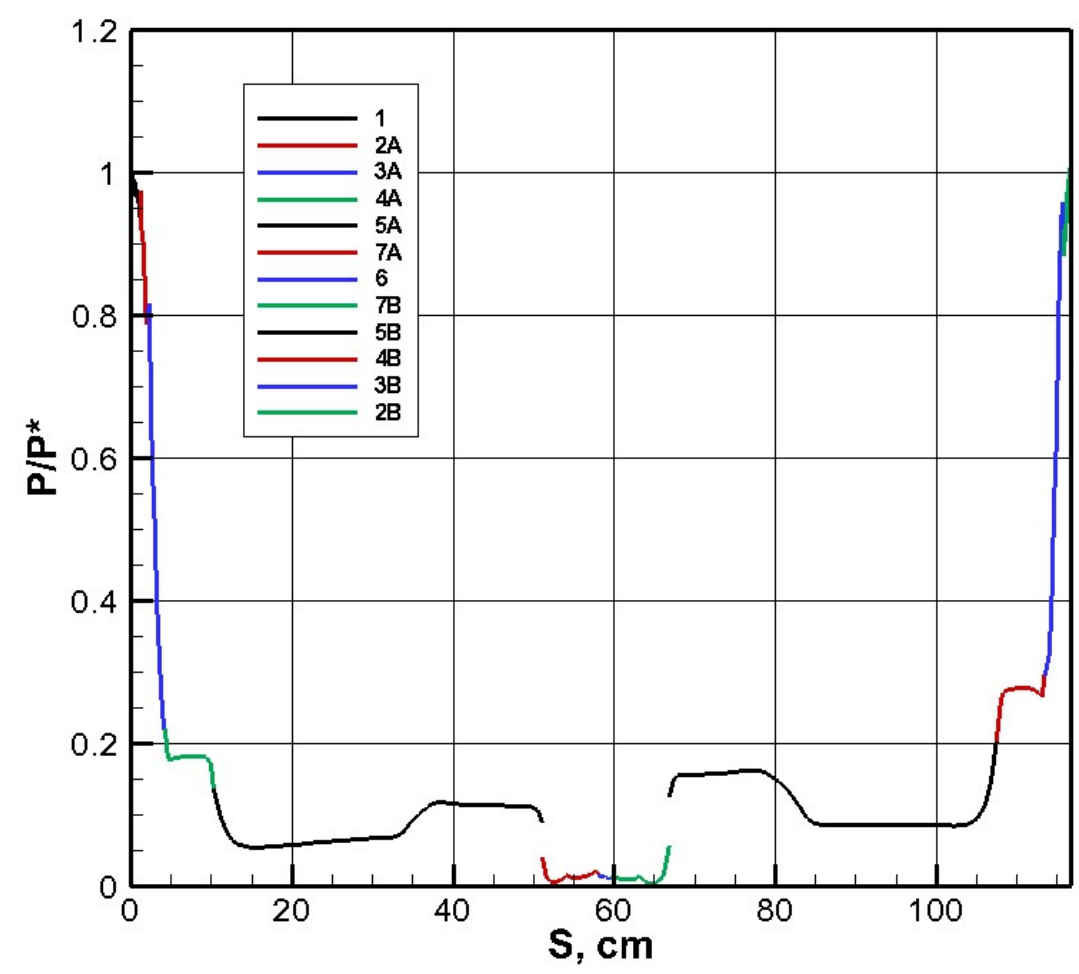

Рис. 15. Распределение давления вдоль поверхности при $\alpha=4^{\circ}$ в плоскости симметрии $z=0$ 


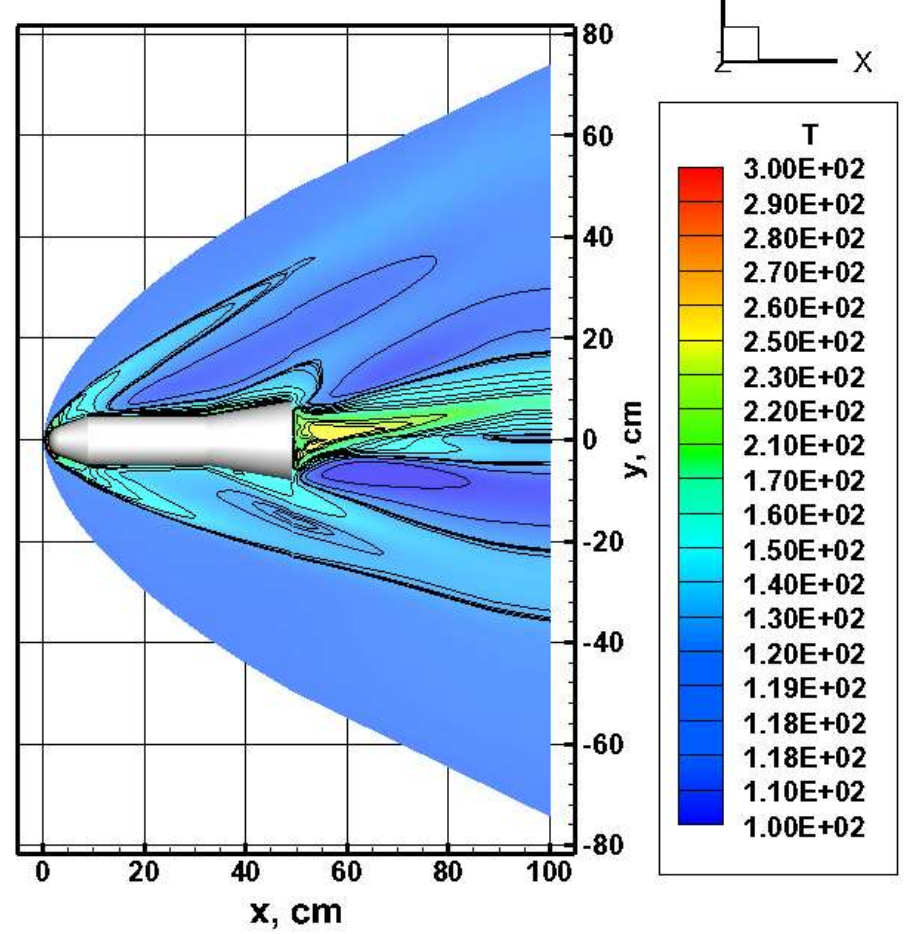

Рис. $16, a$. Поле температуры в К при $\alpha=8^{\circ}$ в плоскости симметрии $z=0$
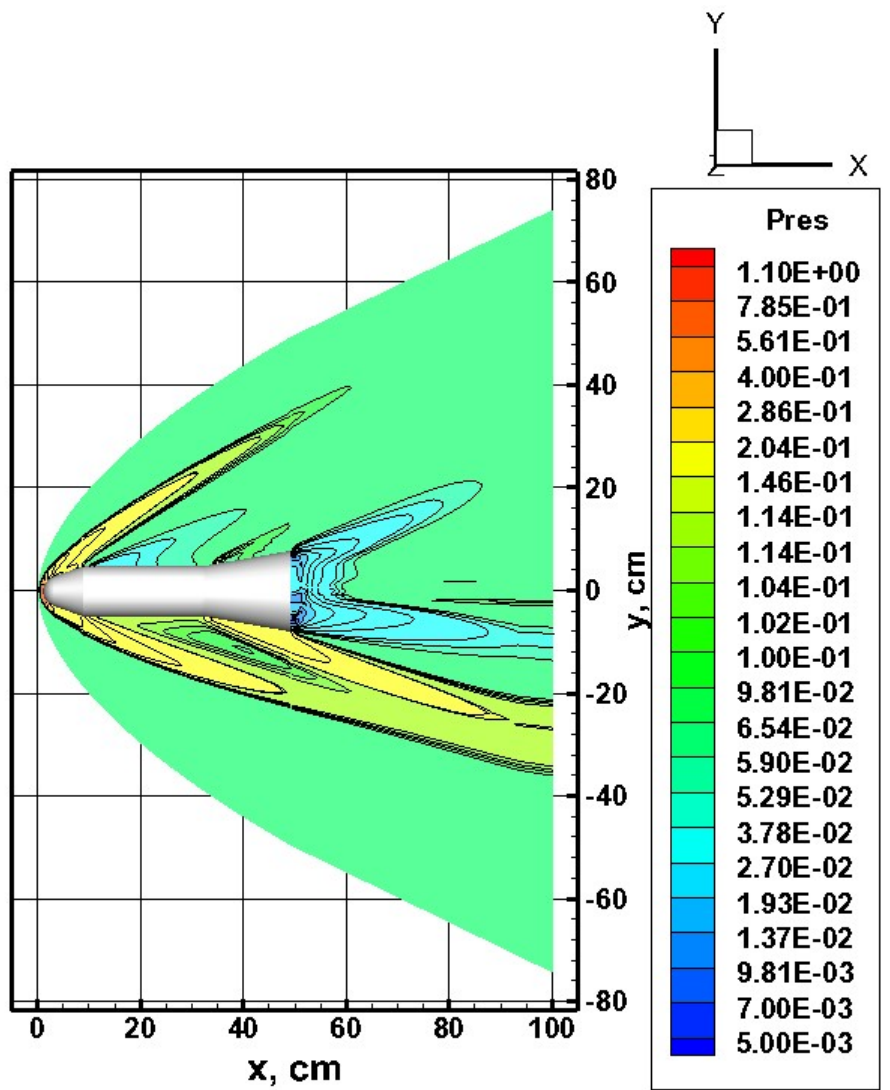

Рис. 16, б. Поле давления ( $\left.\mathrm{PRES}=\frac{p}{p_{0}}\right)$ при $\alpha=8^{\circ}$ в плоскости симметрии $z=0$ 

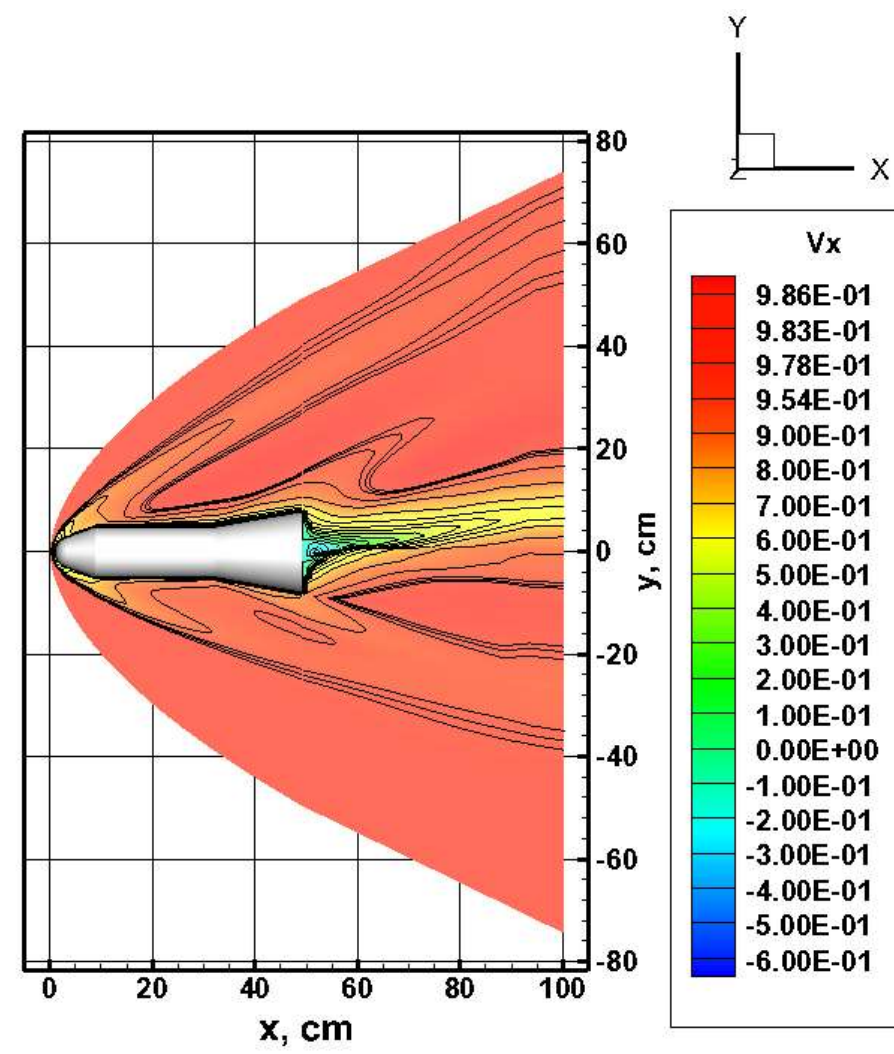

9.86E-01

9.83E-01

9.78E-01

9.54E-01

9.00E-01

8.00E-01

7.00E-01

$6.00 \mathrm{E}-01$

5.00E-01

4.00E-01

3.00E-01

2.00E-01

$1.00 \mathrm{E}-01$

$0.00 \mathrm{E}+00$

$-1.00 \mathrm{E}-01$

-2.00E-01

$-3.00 \mathrm{E}-01$

$-4.00 \mathrm{E}-01$

$-5.00 \mathrm{E}-01$

$-6.00 \mathrm{E}-01$

Рис. 16, в. Поле продольной скорости $\frac{u}{u_{\infty}}$ при $\alpha=8^{\circ}$ в плоскости симметрии $z=0$
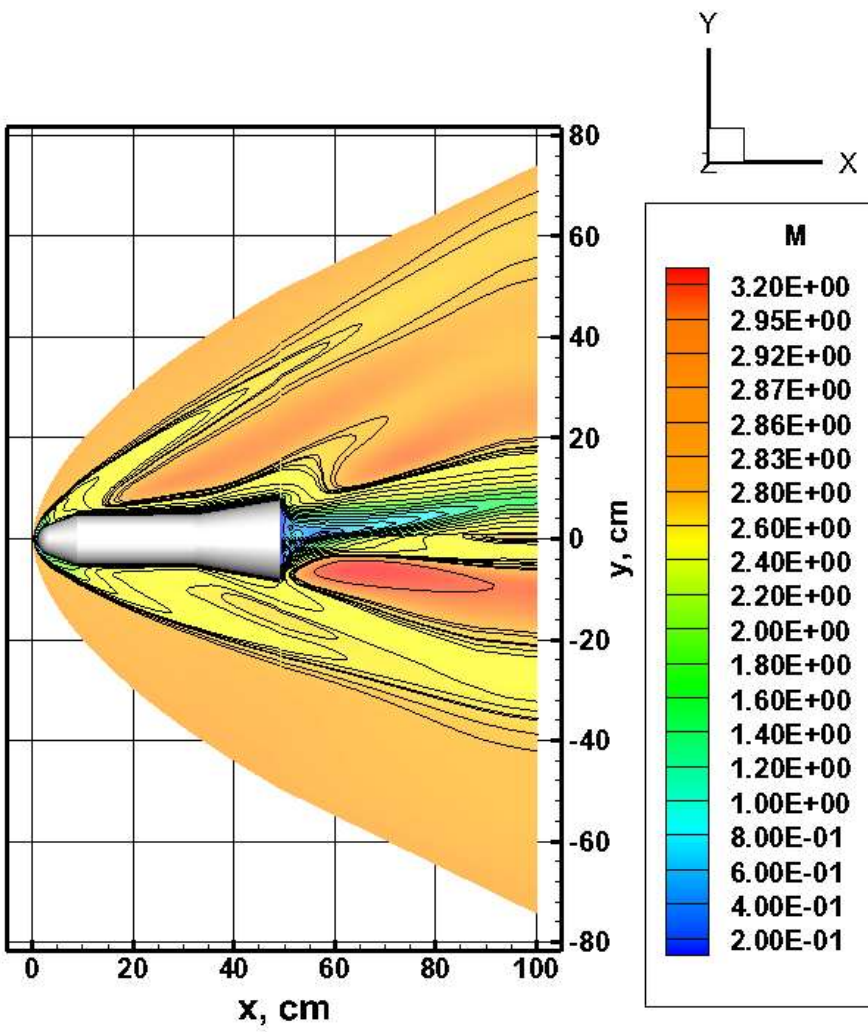

$3.20 \mathrm{E}+00$

2.95E+00

2.92E+00

2.87E+00

$2.86 \mathrm{E}+00$

$2.83 \mathrm{E}+00$

$2.80 \mathrm{E}+00$

$2.60 \mathrm{E}+00$

$2.40 \mathrm{E}+00$

$2.20 \mathrm{E}+00$

$2.00 \mathrm{E}+00$

$1.80 \mathrm{E}+00$

$1.60 \mathrm{E}+00$

$1.40 \mathrm{E}+00$

$1.20 \mathrm{E}+00$

1.00E+00

8. $00 \mathrm{E}-01$

6.00E-01

4. $00 \mathrm{E}-01$

2.00E-01

Рис. 16 , г. Поле чисел Маха при $\alpha=8^{\circ}$ в плоскости симметрии $z=0$ 


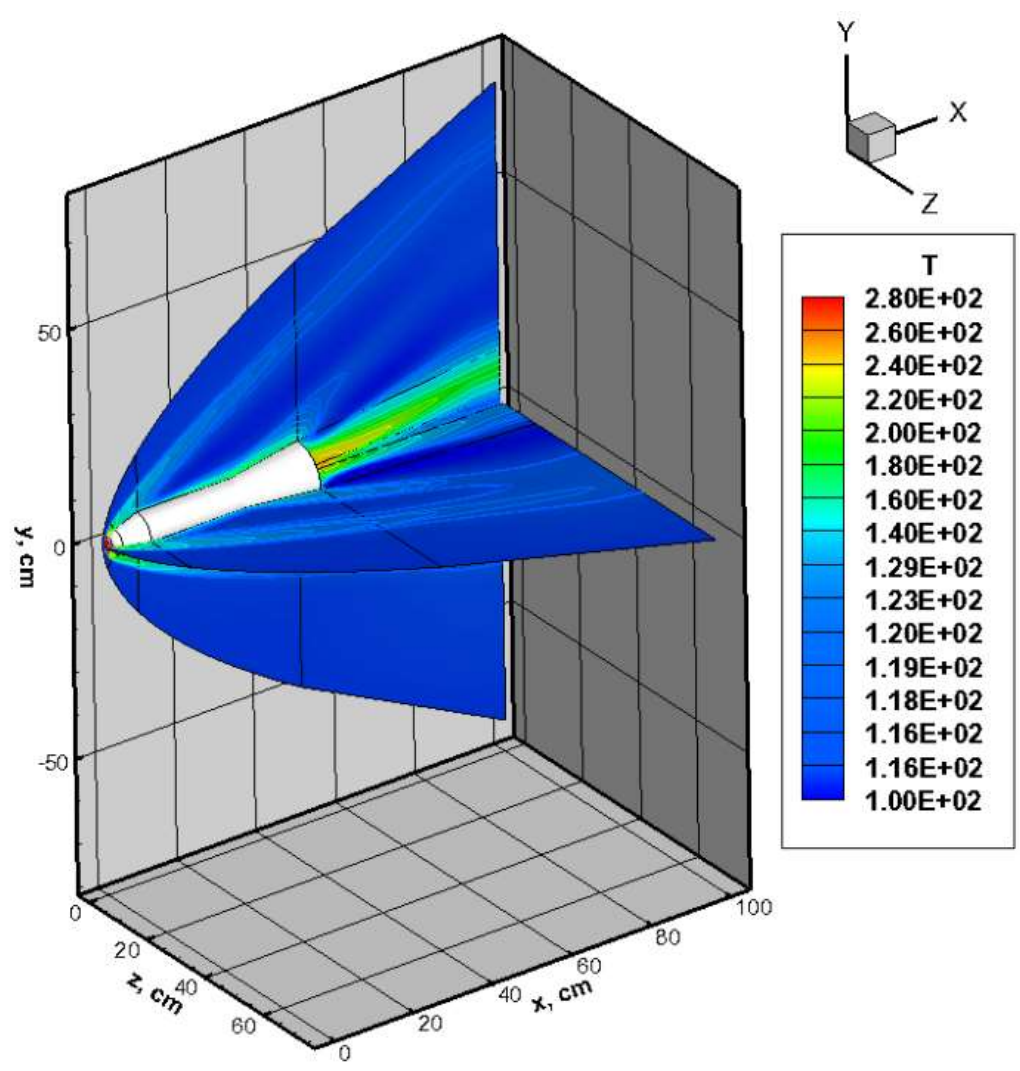

Рис. $16, \partial$. Поле температуры в $\mathrm{K}$ при $\alpha=8^{\circ}$ в плоскостях $y=0$ и $z=0$

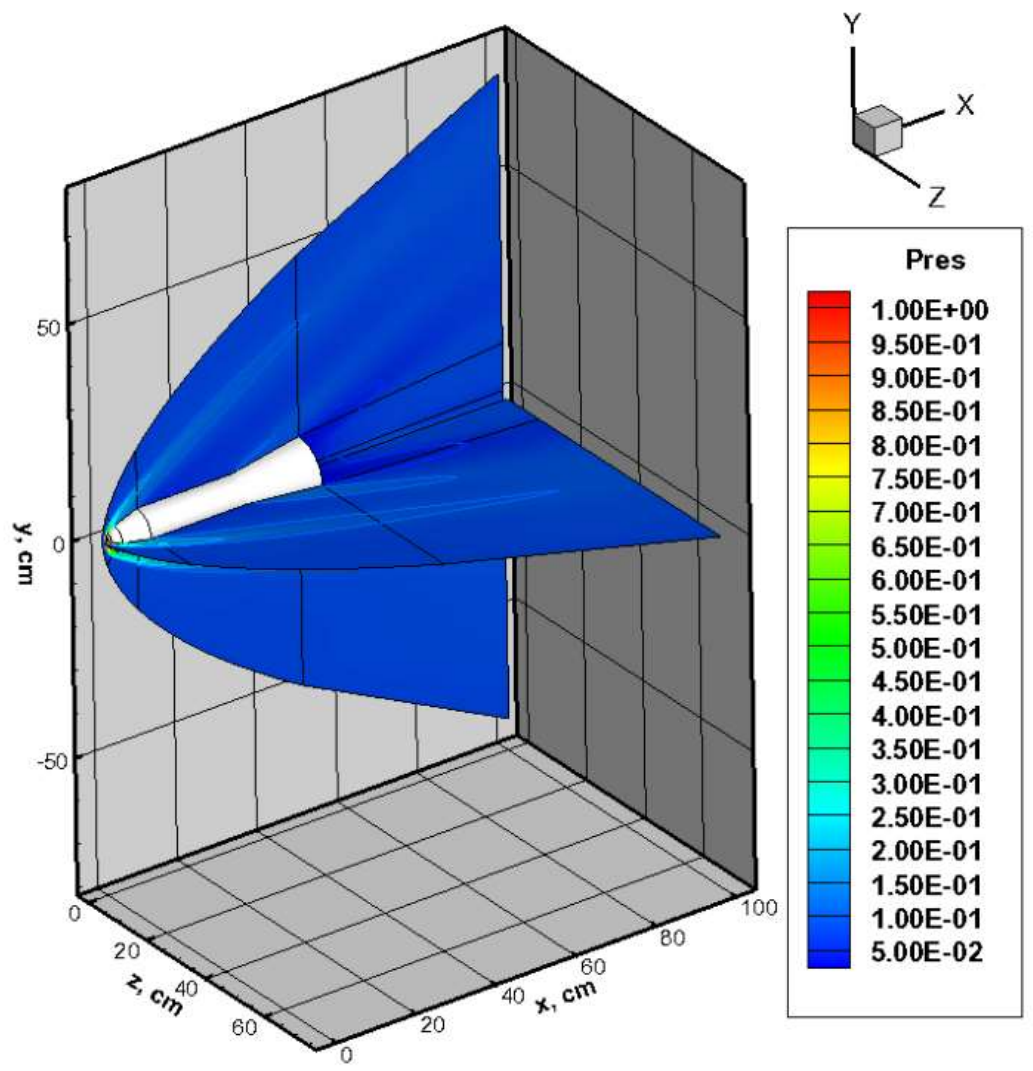

Рис. 16, е. Поле давления ( $\left.\mathrm{PRES}=\frac{p}{p_{0}}\right)$ при $\alpha=8^{\circ}$ в плоскостях $y=0$ и $z=0$ 


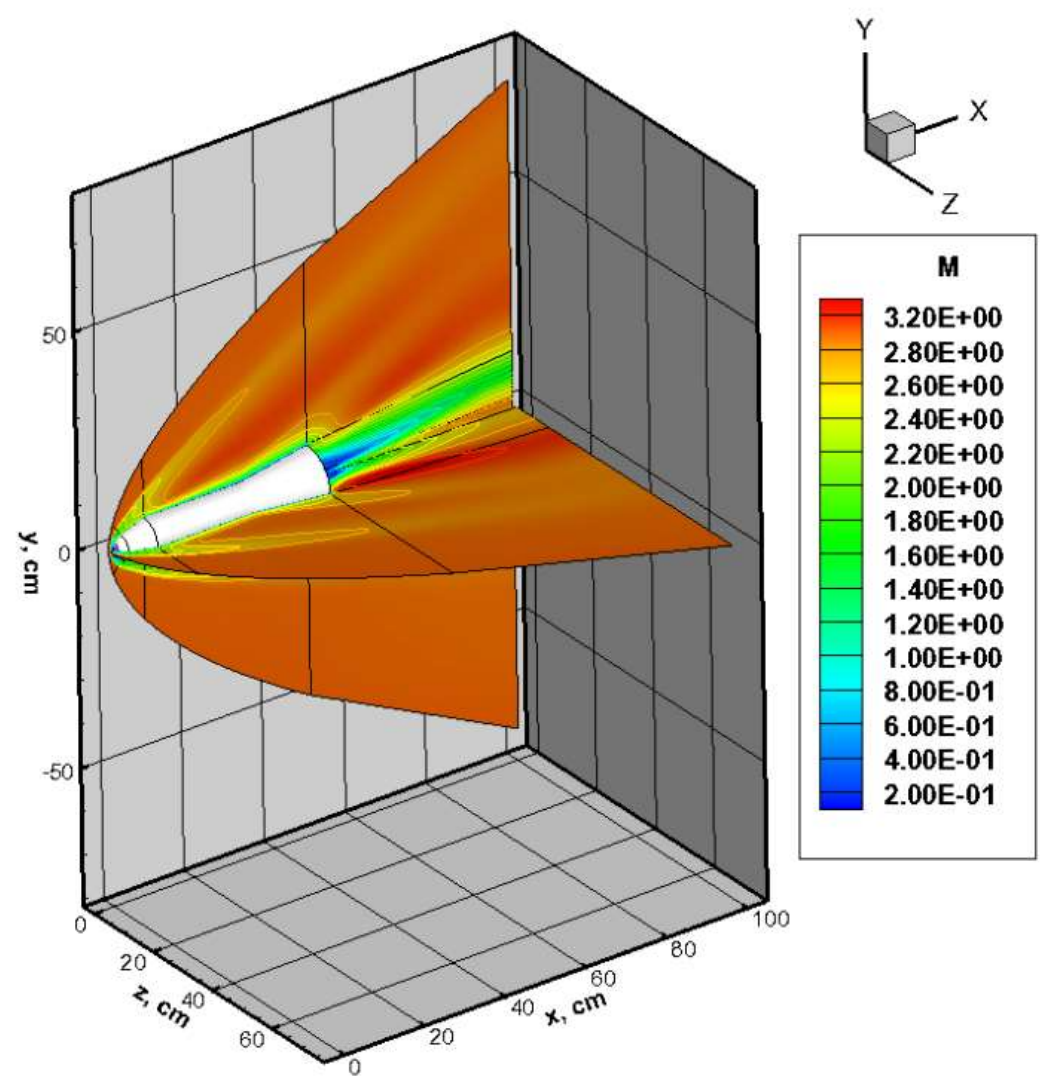

Рис. $16, ж$. Поле чисел Маха при $\alpha=8^{\circ}$ в плоскостях $y=0$ и $z=0$

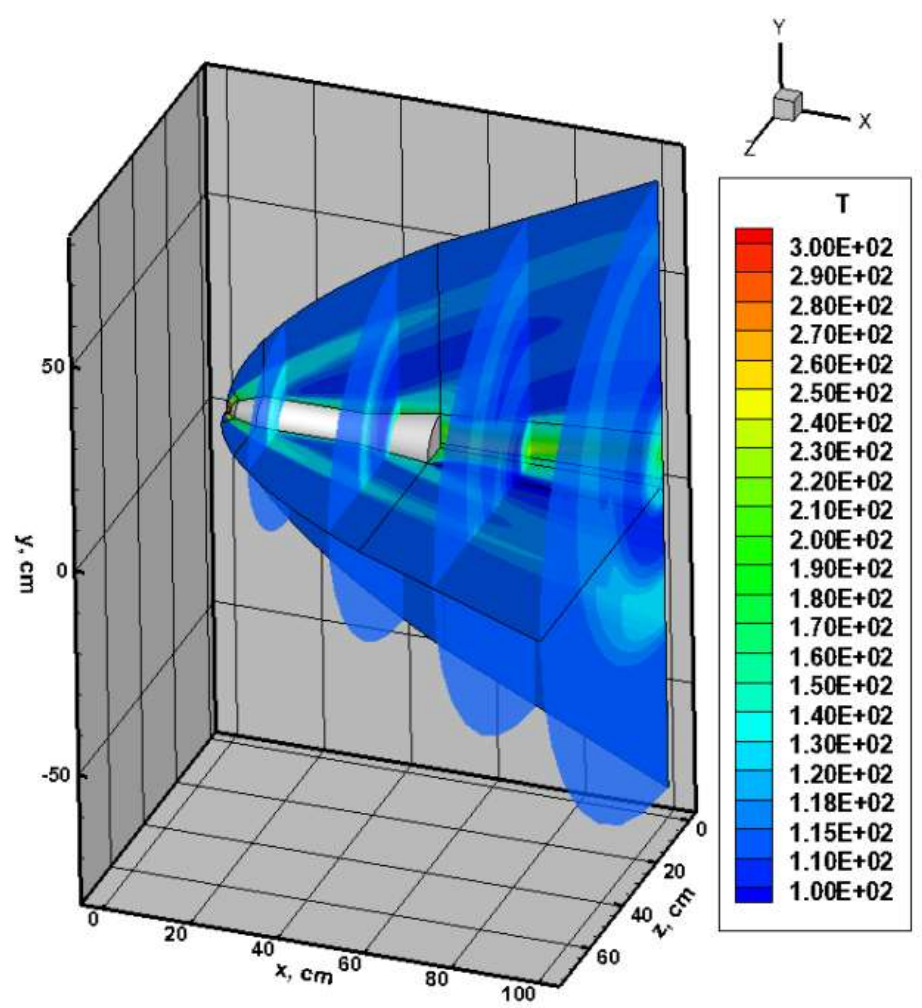

Рис. 16, з. Поле температуры в К при $\alpha=8^{\circ}$ в плоскостях перпендикулярных оси симметрии 


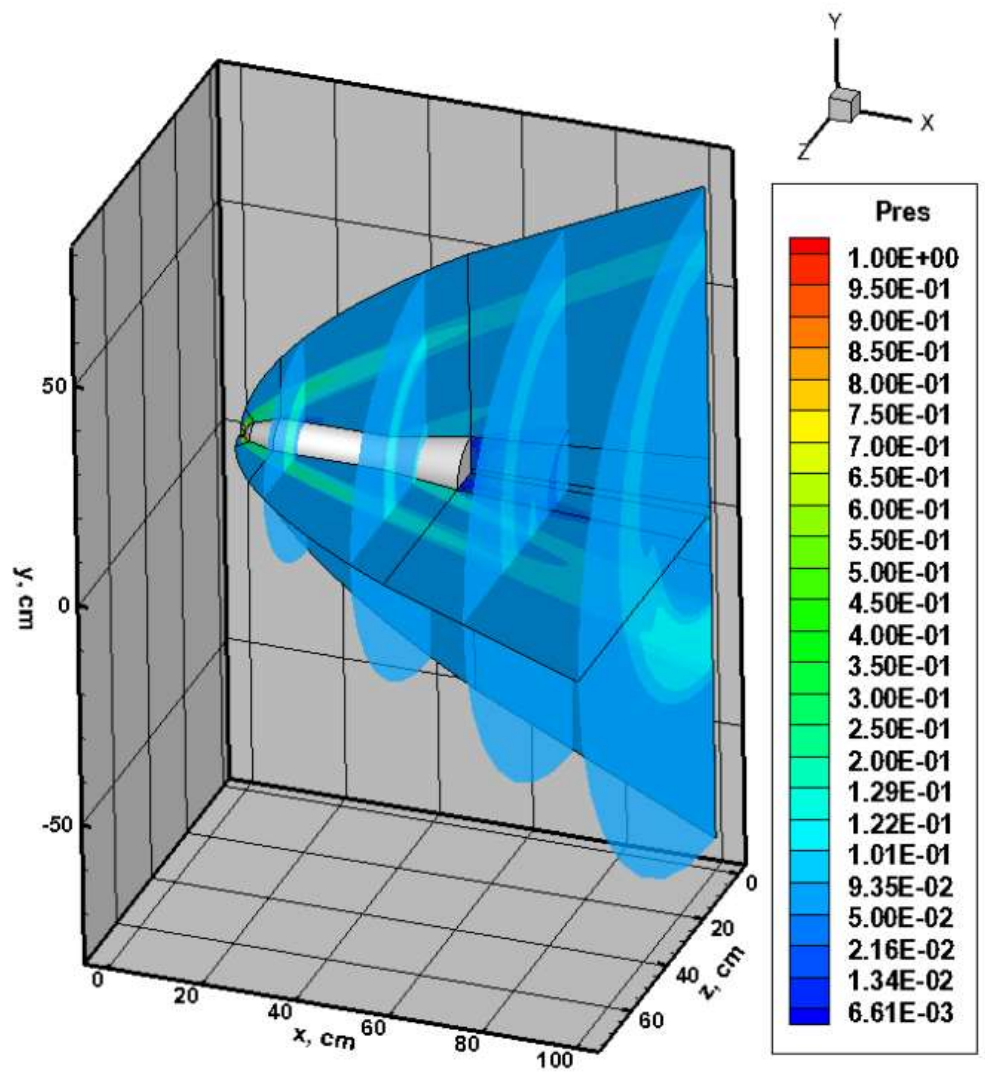

Рис. $16, u$. Поле давления ( $\mathrm{PRES}=\frac{p}{p_{0}}$ ) при $\alpha=8^{\circ}$ в плоскостях перпендикулярных оси симметрии

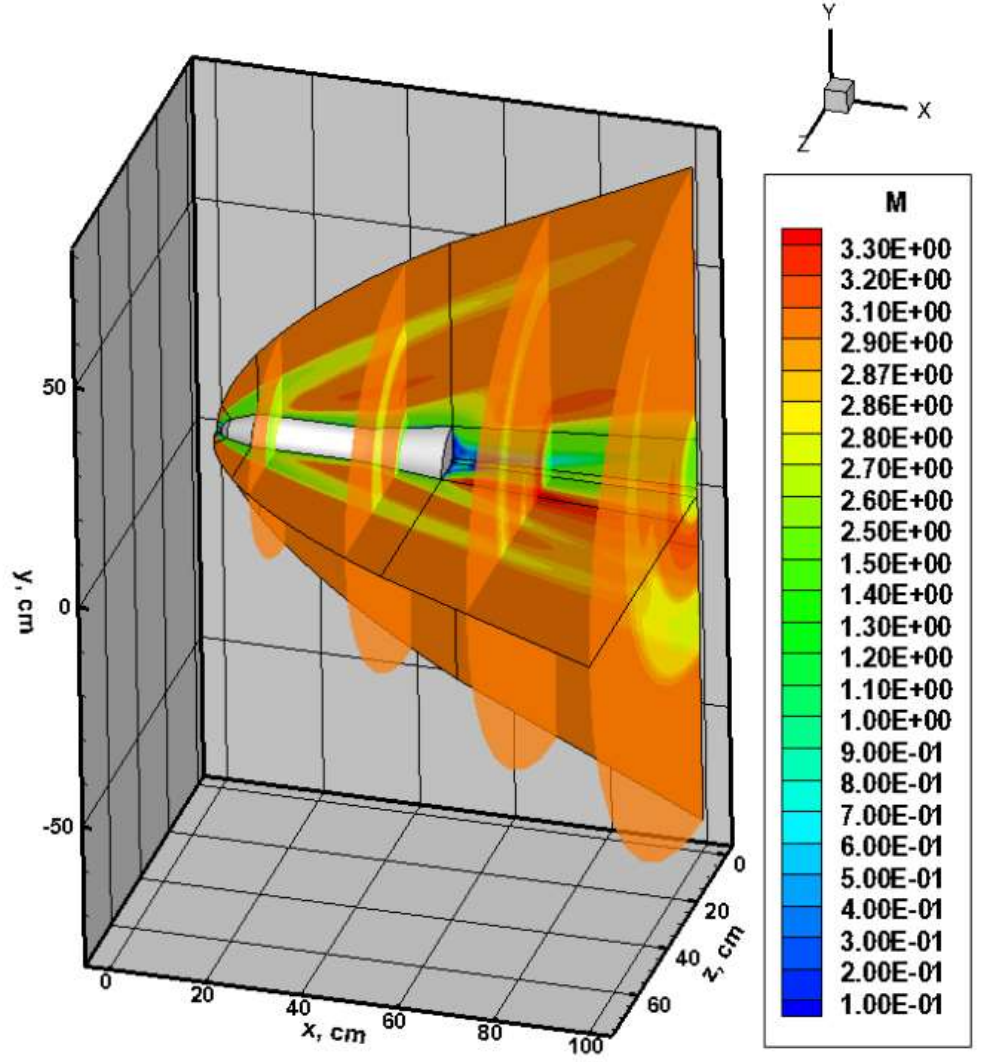

Рис. $16, \kappa$. Поле чисел Маха при $\alpha=8^{\circ}$ в плоскостях перпендикулярных оси симметрии 


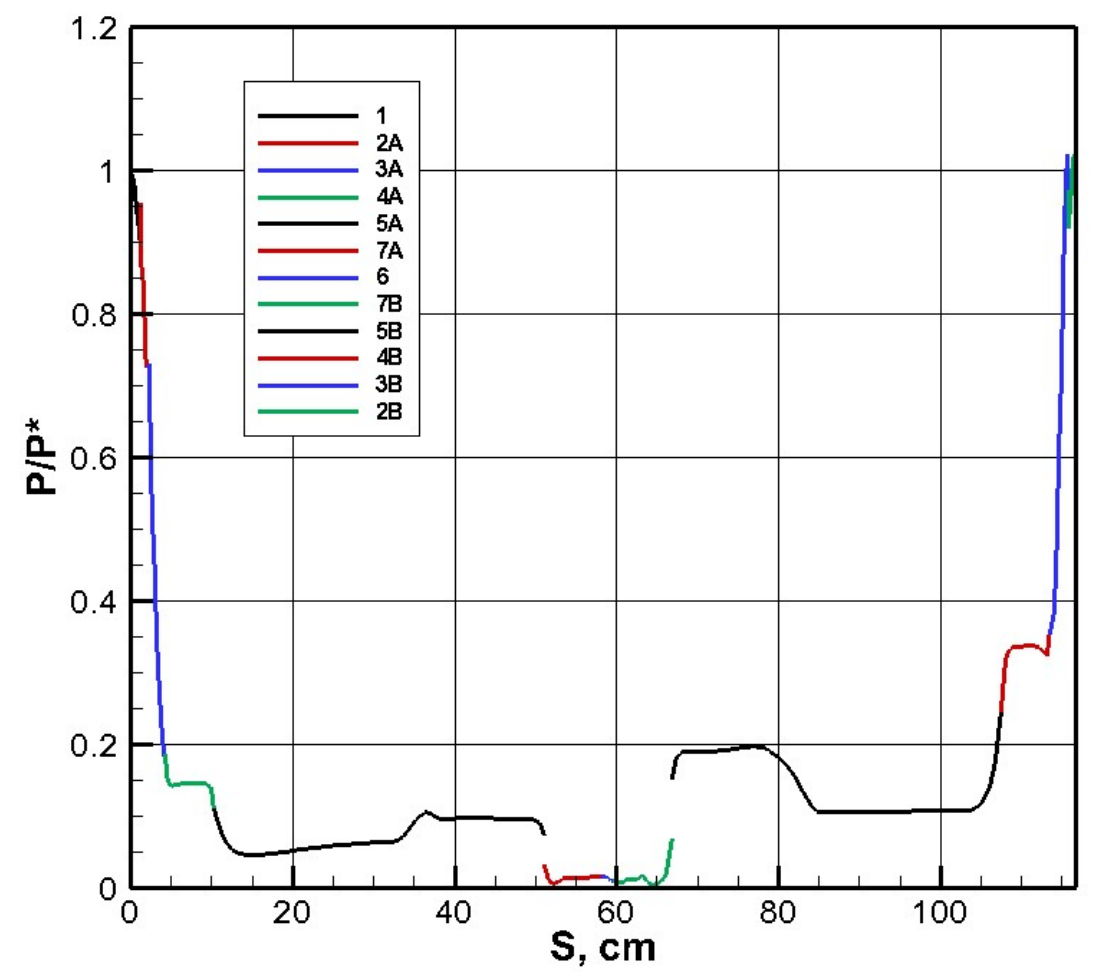

Рис. 17. Распределение давления вдоль поверхности при $\alpha=8^{\circ}$ в плоскости симметрии $z=0$

Как и при анализе результатов, полученных с использованием кода USTFEN, отметим хорошее согласие с экспериментальными данными [30], а также адекватное описание всех характерных областей течения. Таким образом, можно говорить не только о перекрестной верификации указанных компьютерных кодов, но и валидации каждого их них на примере сравнения с экспериментальными данными [30].

\section{7. Заключение}

Представлены результаты тестирования двух авторских компьютерных кодов с целью решения задач фундаментального и прикладного характера аэротермодинамики и аэрофизики гиперзвуковых течений. Первый компьютерный код, USTFEN, основан на использовании неструктурированных тетраэдральных расчетных сеток. Второй компьютерный код, PERAT-3D, основан на использовании структурированных неоднородных многоблочных расчетных сеток. Выполнена валидация указанных компьютерных кодов на примере сравнения с экспериментальными данными по определению аэродинамических характеристик затупленного по сфере кругового цилиндра с юбкой [30]. Выполнено сравнение результатов расчета, полученных с использованием разных компьютерных кодов на структурированной и неструктурированной сетке (так называемая перекрестная верификация компьютерных кодов). В качестве примера показаны различные способы графического представления результатов расчетов.

Помимо расчетных данных в работе приведена формулировка системы уравнений Навье-Стокса в удобном для численного конечно-объемного интегрирования виде и изложен алгоритм численного моделирования на неструктурированных тетраэдральных сетках. Дана формулировка трехмерного конечно-объемного метода для тетраэдральной сетки. Приведены расчетные соотношения, используемые при численном интегрировании на тетраэдральных сетках. Дана формулировка уравнений, реализуемых в компьютерном коде PERAT-3D.

В завершение отметим ряд проблемных вопросов, которые требуют дальнейшей проработки с целью достижения совершенства разрабатываемых авторских компьютерных кодов. Сюда прежде всего относятся задачи упрощения и ускорения построения неструктурирован- 
ных и структурированных расчетных сеток для аэродинамических моделей сложных конфигураций. Второй важной проблемой является ускорение вычислительных процедур, в том числе с использования инновационных подходов распараллеливания и применения новых аппаратных средств.

Работа выполнена по теме государственного задания РАН (№ гос. регистрации ААААА20-120011690135-5) и частично при поддержке РФФИ (грант № 19-01-00515).

\section{Литература}

1. Kimmel R.L., Adamczak D., and Brisbane DSTO-AVD Team HIFiRE-1 Preliminary Aerothermodynamic Experiments 2011 AIAA 2011-3413

2. Stanfield S.A., Kimmel R., and Adamczak D. 2012 HIFiRE-1 Data Analysis: Boundary Layer Transition Experiment During Reentry 2012 AIAA 2012-1087

3. Wadhams T., Mundy E., MacLean M., Holden M. Ground Test Studies of the HIFiRE-1 Transition Experiment. Part 1: Experimental Results. 2008 J. of Spacecraft and Rockets. Vol. 45. No. 5. pp. 1134-1148.

4. MacLean M., Wadhams T., Holden M., Hohnson H. Ground Test Studies of the HIFiRE-1 Transition Experiment. Part 2: Computational Analysis. 2008 J. of Spacecraft and Rockets. Vol. 45. No. 5. pp. 1149-1164.

5. Holden M.S., Wadhams T.P., Harvey J.K., Candler G.V. Comparison between Measurements in Regions of Laminar Shock Wave Boundary Layer Interaction in Hypersonic Flows with Navier-Stokes and DSMC Solutions. RTO-TR-AVT-007-03.

6. Candler G.V., Nompelis I., Druguet M.-C., Holden M.S., Wadhams T.P., Boyd I.D. CFD Validation for Hypersonic Flight: Hypersonic Double-Cone Flow Simulation. AIAA 2002-0581. 14 p.

7. MacLean M., Holden M. Validation and Comparison of WIND and DPLR Results for Hypersonic, Laminar Problems. AIAA 2004-0529. 17 p.

8. Moss J.N., Olejniczak J. Shock-Wave/Boundary-Layer Interactions in Hypersonic Low Density Flows. AIAA 98-2668. 19 p.

9. Wright M.J., Sinha K., Olejniczak J., Candler G.V. Numerical and Experimental Investigation of Double-Cone Shock Interactions. AIAA Journal. 2000. Vol.38. No.12. pp. 2268-2276.

10. Harvey J.K., Holden M.S., Wadhams T.P. Code Validation Study of Laminar Shock/Boundary Layer and Shock/Shock Interactions in Hypersonic Flow. AIAA 2001-1031. 27 p.

11. Боровой В.Я., Егоров И.В., Скуратов А.С., Струминская И.В. Ламинарный теплообмен острых и притупленных пластин в гиперзвуковом потоке воздуха//Изв. РАН МЖГ. 2005. №1. С.168-180.

12. Surzhikov S.T. Validation of computational code UST3D by the example of experimental aerodynamic data. Journal Phys.: Conf. Ser. Journal of Physics Conference Series 815(1):012023. 2017. DOI: 10.1088/1742-6596/815/1/012023

13. Surzhikov S.T. Comparative Analysis of the Results of Aerodynamic Calculation of a Spherical Blunted Cone on a Structured and Unstructured Grid// IOP Conf. Series: Journal of Physics: Conf. Series. 1250 (2019) 012007 doi:10.1088/1742-6596/1250/1/012007

14. Суржиков С.Т. Радиационная газовая динамика спускаемых космических аппаратов. Многотемпературные модели. М.: ИПМех РАН. 2013. 706 с.

15. Суржиков С.Т. Пространственная задача радиационной газовой линамики командного модуля Аполлон-4 при сверхорбитальном входе в атмосферу// Изв. РАН МЖГ, 2018, № 2, С. 149-160.

16. Суржиков С.Т. Анализ экспериментальных данных по конвективному нагреву модели марсианского спускаемого аппарата с использованием алгебраических моделей турбулентности // Изв. РАН МЖГ, 2019, № 6, С.129-140. 
17. Surzhikov S.T. Calculation analysis of the experimental data of HIFiRE-I using the computer code NERAT-2D IOP Conf. Series: Journal of Physics: Conf. Series 1009 (2018) 012001 doi :10.1088/1742-6596/1009/1/012001

18. Суржиков С.Т. Численное моделирование ударно-волнового взаимодействия с ламинарным пограничным слоем при гиперзвуковом обтекании моделей с изломом образующей//Физико-химическая кинетика в газовой динамике. 2019. Т. 20, вып. 4. http://chemphys.edu.ru/issues/2019-20-4/articles/892/

19. Железнякова А.Л., Суржиков С.Т. Применение метода расщепления по физическим процессам для расчета гиперзвукового обтекания пространственной модели летательного аппарата сложной формы// ТВT. 2013. Т. 51. № 6. С. 897-911.

20. Железнякова А.Л., Суржиков С.Т. Расчет гиперзвукового обтекания тел сложной формы на неструктурированных тетраэдральных сетках с использованием схемы AUSM// TBT. 2014. Т. 52. № 2. С. 283-293.

21. Белоцерковский О.М., Давыдов Ю.М. Метод крупных частиц в газовой динамике. М.: Наука, 1982. $391 \mathrm{c}$.

22. Марчук Г.И. Методы расщепления. М.: Наука, 1988. 263 с.

23. Ковеня В.М., Яненко Н.Н. Метод расщепления в задачах газовой динамики// Новосибирск: Наука, 1981. 304 с.

24. Крючкова А.С. Моделирование сверхзвукового обтекания баллистических моделей в программном коде UST3D//Физико-химическая кинетика в газовой динамике. 2018. Т. 19, вып. 4. http://chemphys.edu.ru/issues/2018-19-4/articles/783/

25. Яцухно Д.С. Численное моделирование аэродинамики волнолетов построенных на скачках уплотнения различной формы//Физико-химическая кинетика в газовой динамике. 2020. Т. 21, вып. 1. http://chemphys.edu.ru/issues/2020-21-1/articles/881/

26. Сильвестров П.В., Суржиков С.Т. Расчет аэротермодинамики высокоскоростного летательного аппаратаX-43 с использованием компьютерных кодов UST3D и UST3D-AUSMPW//Физикохимическая кинетика в газовой динамике. 2019. Т. 20, вып. 4. http://chemphys.edu.ru/issues/201920-4/articles/865/

27. Yatsukhno D.S. Computational study of the waverider aerothermodynamics by the UST3D computer code // J. of Physics: Conference Series. 2018. Vol. 1009. No. 012002. https://doi.org/10.1088/1742$\underline{6596 / 1009 / 1 / 012002}$

28. Яцухно Д.С., Суржиков С.Т. Метод расщепления по физическим процессам в задаче моделирования обтекания перспективного высокоскоростного летательного аппарата//Вестник МГТУ им. Н.Э. Баумана. Сер. «Машиностроение». 2018. № 1. С. 20-33.

29. З Зинин К.М., Панасенко А.В., Суржиков С.T. Валидация компьютерного кода UST3D на примере расчета аэродинамических характеристик летательного аппарата X-34 при различных парамет$\mathrm{pax} / /$ Физико-химическаякинетикавгазовойдинамике.2019.Т. 20, вып. 4.

http://chemphys.edu.ru/issues/2019-20-4/articles/882/

30. Gray J.D., Lindsay J.J. Force tests of standard hypervelocity ballistic models HB-1 and HB-2 at Mach 1.5 to 10//AEDC-TDR S3 -137. 1963.

31. Будник А.П., Алексеева И.В., Сипачев А.В. Частное сообщение. Август 2010 г.

\section{References}

1. Kimmel, R. L., Adamczak, D., and Brisbane DSTO-AVD Team, "HIFiRE-1 Preliminary Aerothermodynamic Experiments,"41st AIAA Fluid Dynamics Conference and Exhibit, AIAA Paper 2011-3413, Jan. 2011. 
2. Stanfield, S. A., Kimmel, R., and Adamczak, D., "HIFiRE-1 Data Analysis: Boundary Layer Transition Experiment During Reentry," 50th AIAA Aerospace Sciences Meeting, AIAA Paper 2012-1087, Jan. 2012.

3. Wadhams, T., Mundy, E., MacLean, M., Holden, M., "Ground Test Studies of the HIFiRE-1 Transition Experiment. Part 1: Experimental Results," J. of Spacecraft and Rockets, Vol. 45, No. 5, NovemberDecember 2008, pp. 1134-1148.

4. MacLean, M., Wadhams, T., Holden, M., Hohnson, H., "Ground Test Studies of the HIFiRE-1 Transition Experiment. Part 2: Computational Analysis," J. of Spacecraft and Rockets, Vol. 45, No. 5, November-December 2008, pp. 1149-1164.

5. Holden, M. S., Wadhams, T. P., Harvey, J. K., Candler, G. V., "Comparison between Measurements in Regions of Laminar Shock Wave Boundary Layer Interaction in Hypersonic Flows with Navier - Stokes and DSMC Solutions," RTO-TR-AVT-007-03.

6. Candler, G. V., Nompelis, I., Druguet, M.-C., Holden, M. S., Wadhams, T. P., Boyd, I. D., "CFD Validation for Hypersonic Flight: Hypersonic Double-Cone Flow Simulation," 40th AIAA Aerospace Sciences Meeting \& Exhibit, AIAA Paper 2002-0581, Jan. 2002.

7. MacLean, M., Holden, M., "Validation and Comparison of WIND and DPLR Results for Hypersonic, Laminar Problems," 42nd AIAA Aerospace Sciences Meeting \& Exhibit, Reno, NV., AIAA Paper 20040529, Jan. 2004.

8. Moss, J.N., Olejniczak, J., "Shock-Wave/Boundary-Layer Interactions in Hypersonic Low Density Flows," $7^{\text {th }}$ AIAA/ASME Joint Thermophysics and Heat Transfer Conference, AIAA 98-2668, June 1998.

9. Wright, M. J., Sinha, K., Olejniczak J., Candler, G. V., "Numerical and Experimental Investigation of Double-Cone Shock Interactions," AIAA Journal, Vol. 38, No. 12, 2000, pp. 2268-2276. doi: $10.2514 / 2.918$

10. Harvey, J. K., Holden, M. S., Wadhams, T. P., "Code Validation Study of Laminar Shock/Boundary Layer and Shock/Shock Interactions in Hypersonic Flow," 39th Aerospace Sciences Meeting and Exhibition, AIAA 2001-1031, Jan. 2001.

11. Borovoj, V. Ja., Egorov, I. V., Skuratov, A. S., Struminskaja, I. V., "Laminarnyj teploobmen ostryh i prituplennyh plastin v giperzvukovom potoke vozduha," Izv. RAN MZhG, No. 1, 2005, pp. 68-180.

12. Surzhikov, S. T., "Validation of computational code UST3D by the example of experimental aerodynamic data," Journal of Physics: Conference Series, Vol. 815, No. 012023, 2017. doi: 10.1088/1742-6596/815/1/012023

13. Surzhikov, S. T., "Comparative Analysis of the Results of Aerodynamic Calculation of a Spherical Blunted Cone on a Structured and Unstructured Grid," Journal of Physics: Conf. Series, Vol. 1250, No. 012007, 2019. doi:10.1088/1742-6596/1250/1/012007

14. Surzhikov, S. T., Radiacionnaja gazovaja dinamika spuskaemyh kosmicheskih apparatov. Mnogotemperaturnye modeli (Radiationgas dynamics of reentry spacecraft. Multi-temperature models), M.: IPMeh RAN, 2013.

15. Surzhikov, S. T.,"Prostranstvennaja zadacha radiacionnoj gazovoj linamiki komandnogo modulja Apollon-4 pri sverhorbital'nom vhode v atmosferu," Izv. RAN MZhG, No. 2, 2018, pp. 149-160.

16. Surzhikov, S. T., “Analiz jeksperimental'nyh dannyh po konvektivnomu nagrevu modeli marsianskogo spuskaemogo apparata $\mathrm{s}$ ispol'zovaniem algebraicheskih modelej turbulentnosti," Izv. RAN MZhG, No. 6, 2019, pp.129-140.

17. Surzhikov, S. T., "Calculation analysis of the experimental data of HIFiRE-I using the computer code NERAT-2D," Journal of Physics: Conference Series, Vol. 1009, No. 012001, 2018. doi :10.1088/1742-6596/1009/1/012001 
18. Surzhikov, S. T., "Chislennoe modelirovanie udarno-volnovogo vzaimodejstvija s laminarnym pogranichnym sloem pri giperzvukovom obtekanii modelej s izlomom obrazujushhej," Fiziko-himicheskaja kinetika v gazovoj dinamike (Phisical-Chemical Kinetics in Gas Dynamics), Vol. 20, No. 4, 2019. http://chemphys.edu.ru/issues/2019-20-4/articles/892/

19. Zheleznjakova, A. L., Surzhikov, S. T., "Primenenie metoda rasshheplenija po fizicheskim processam dlja rascheta giperzvukovogo obtekanija prostranstvennoj modeli letatel'nogo apparata slozhnoj formy," TVT, Vol. 51, No. 6, 2013, pp. 897-911.

20. Zheleznjakova, A. L., Surzhikov, S. T., "Raschet giperzvukovogo obtekanija tel slozhnoj formy na nestrukturirovannyh tetrajedral'nyh setkah s ispol'zovaniem shemy AUSM," TVT, Vol. 52, No. 2, 2014, pp. 283-293.

21. Belocerkovskij, O. M., Davydov, Ju. M., Metod krupnyh chastic v gazovoj dinamike (The Large particle method in gas dynamics), M.: Nauka, 1982.

22. Marchuk, G. I., Metody rasshheplenija (Splitting method), M.: Nauka, 1988.

23. Kovenja, V. M., Janenko, N. N. Metod rasshheplenija $v$ zadachah gazovoj dinamiki (The Splitting method in gas dynamics problems), Novosibirsk: Nauka, 1981.

24. Krjuchkova, A. S., "Modelirovanie sverhzvukovogo obtekanija ballisticheskih modelej v programmnom kode UST3D," Fiziko-himicheskaja kinetika v gazovoj dinamike (Phisical-Chemical Kinetics in Gas Dynamics), Vol. 19, No. 4, 2018. http://chemphys.edu.ru/issues/2018-19-4/articles/783/

25. Jacuhno, D. S., "Chislennoe modelirovanie ajerodinamiki volnoletov postroennyh na skachkah uplotnenija razlichnoj formy," Fiziko-himicheskaja kinetika v gazovoj dinamike (Phisical-Chemical Kinetics in Gas Dynamics), Vol. 21, No. 1, 2020. http://chemphys.edu.ru/issues/2020-21-1/articles/881/

26. Sil'vestrov, P. V., Surzhikov, S. T., "Raschet ajerotermodinamiki vysokoskorostnogo letatel'nogo apparata X-43 s ispol'zovaniem komp'juternyh kodov UST3D i UST3D-AUSMPW," Fizikohimicheskaja kinetika v gazovoj dinamike (Phisical-Chemical Kinetics in Gas Dynamics), Vol. 20, No. 4, 2019. http://chemphys.edu.ru/issues/2019-20-4/articles/865/

27. Yatsukhno, D. S., "Computational study of the waverider aerothermodynamics by the UST3D computer code," Journal of Physics: Conference Series. 2018. Vol. 1009. No. 012002.

doi: 10.1088/1742-6596/1009/1/012002

28. Yatsukhno, D. S., Surzhikov, S. T., "Metod rasshheplenija po fizicheskim processam v zadache modelirovanija obtekanija perspektivnogo vysokoskorostnogo letatel'nogo apparata," Vestnik MGTU im. N. Je. Baumana. Ser. «Mashinostroenie», No. 1, 2018, pp. 20-33.

29. Zinin, K. M., Panasenko, A. V., Surzhikov, S. T., "Validacija komp'juternogo koda UST3D na primere rascheta ajerodinamicheskih harakteristik letatel'nogo apparata X-34 pri razlichnyh parametrah," Fiziko-himicheskaja kinetika v gazovoj dinamike (Phisical-Chemical Kinetics in Gas Dynamics), Vol. 20, No. 4, 2019. http://chemphys.edu.ru/issues/2019-20-4/articles/882/

30. Gray, J. D., Lindsay, J. J., "Force tests of standard hypervelocity ballistic models HB-1 and HB-2 at Mach 1.5 to 10," AEDC-TDR S3 -137, 1963.

31. Budnik, A. P., Alekseeva, I.V., Sipachev, A. V. Private message. August 2010. 